\title{
A Common Thread: the pairing interaction for the unconventional superconductors
}

\author{
D. J. Scalapind* \\ Department of Physics, University of California, Santa Barbara, CA 93106-9530 USA
}

The structures, the phase diagrams, and the appearance of a neutron resonance signaling an unconventional superconducting state provide phenomenological evidence relating the cuprates, the Fe-pnictides/chalcogenides as well as some heavy fermion and actinide materials. Single- and multi-band Hubbard models have been found to describe a number of the observed properties of these materials so that it is reasonable to examine the origin of the pairing interaction in these models. In this review, based on the experimental phenomenology and studies of the pairing interaction for Hubbard-like models, it is proposed that spin-fluctuation mediated pairing is the common thread linking a broad class of superconducting materials. 


\section{CONTENTS}

I. Introduction

II. Common Features of a Class of Unconventional Superconductors
A. Structures
B. Phase diagrams
C. Coexistence and interplay of antiferromagnetism and superconductivity
D. A neutron spin resonance

III. Models
A. The cuprates
B. The Fe-pnictides
C. The heavy fermion materials

22

IV. The Pairing Interaction

A. The single band Hubbard model

B. The bilayer Hubbard model

47

C. Multi-orbital models

A. The Structure of Two Pairing Interactions

1. The Electron-phonon Screened Coulomb Pairing Interaction

2. The Spin-Fluctuation Exchange Pairing Interaction

Acknowledgments

References

\section{INTRODUCTION}

Fisk et al. ㄴ $^{1}$ have noted that a striking aspect of superconducting materials is the "remarkable amount of phase space they inhabit: superconductivity is everywhere but sparse. So the central question in superconductivity and the search for new superconducting materials 
is whether there is anything common to the known superconductors." This review addresses this question by examining common features of the cuprate and iron superconductors as well as some heavy fermion and actinide superconductors to see what they tell us about the pairing mechanism in these materials. ${ }^{2}$

We begin in Sec. II by looking at the crystal structures, the phase diagrams, the coexistence and interplay of antiferromagnetism and superconductivity and a neutron scattering spin resonance which is observed in the superconducting phase. One finds that these materials come in families which have quasi-2D layers containing square arrays of $d$ - or $f$-electron cations. Their temperature-doping and magnetic field phase diagrams show antiferromagnetism in close proximity, or in some cases coexisting, with superconductivity. A variety of experiments show that the antiferromagnetism and superconductivity are strongly coupled. A spin resonance peak, which is observed in inelastic neutron scattering experiments in the superconducting phase, provides evidence of unconventional pairing. The similarity of the structures, the phase diagrams, the interplay of antiferromagnetism and superconductivity, and the unconventional nature of the superconductivity seen in these materials suggest they share a common underlying physics.

Sec. III contains a discussion of models that have been used to describe these materials. These are minimal models in which the cuprates are described by a single-band 2-dimensional Hubbard model while the heavy fermion and Fe materials involve orbital degenerate multiband models. Various numerical calculations as well as approximate analytic calculations find that these models exhibit a number of phenomena which are experimentally observed in these materials. In particular, the close proximity of an antiferromagnetic or spin-densitywave phase to an unconventional $d$-wave or sign changing $s$-wave superconducting phase is found to be a common feature. A second important common feature is the dual character of the $3 d$ or $4 f$ electrons in these models. These electrons are involved in both the magnetism and the superconductivity. The models can exhibit behavior ranging from local moments and insulating antiferromagnetic order to itinerant magnetism, stripes and superconductivity. Furthermore the models show the close relationship between $d$-wave and $s^{ \pm}$-wave pairing.

Motivated by this, the momentum, frequency and orbital dependence of the interaction which is responsible for pairing in these models is examined in Section IV. The "same electrons" that are associated with the magnetism and superconductivity are found to give rise to a spin-fluctuation mediated pairing interaction. The short range near-neighbor antifer- 
romagnetic fluctuations give rise to a sign changing gap $(\operatorname{Sgn} \Delta(k+Q)=-\operatorname{Sgn} \Delta(k))$ for large momentum transfers. Appendix A contains a comparison of the traditional electronphonon-Coulomb pairing interaction with this interaction. Based on the experimental phenomenology and the analysis of the models, it is proposed that this spin-fluctuation pairing interaction is the common thread that links this class of unconventional superconducting materials. Although the organic Bechgaard salts ${ }^{3}$ will not be discussed, they clearly are also part of this class of materials $\underline{\underline{4}} \underline{\underline{6}}$. Section $\mathrm{V}$ contains a brief summary and an outlook regarding the guidance this brings to the search for higher $T_{c}$ materials.

\section{COMMON FEATURES OF A CLASS OF UNCONVENTIONAL SUPERCONDUCTORS}

In this section we begin by looking at similarities in the structures and the phase diagrams of some heavy fermion, cuprate and iron-based superconductors. Following this, experimental evidence of the interplay of antiferromagnetism and superconducting and the dominant role of spin-fluctuation scattering in these materials will be discussed. The section concludes with an experimental definition of what we will call "unconventional superconductors" in this review.

\section{A. Structures}

As illustrated ${ }^{7}$ in Figs. 1, 4 , these materials come in families and the common structural element is a quasi 2-dimensional layer with metallic $d$ or $f$ cations arranged on a nominally square planar set of lattice sites. Surrounding these sites are an array of ligand anions which provide a local crystal field and a hybridization network. Three members of the heavy fermion $\mathrm{CeIn}_{3}$ family are shown in Fig. 1. On the left is the unit cell of the socalled infinite layered $\left(T_{c} \sim 0.2 \mathrm{~K}\right)$ material in which $\mathrm{CeIn}_{3}$ layers are stacked one on top of another ${ }^{\underline{8}}$. The middle structure consists of a similar stack of $\mathrm{CeIn}_{3}$ layers in which a $\mathrm{CoIn}_{2}$ layer is inserted after every two $\mathrm{CeIn}_{3}$ layers. This is called a 218 structure corresponding to $\left(\mathrm{CeIn}_{3}\right)_{2}\left(\mathrm{CoIn}_{2}\right)_{1}=\mathrm{Ce}_{2} \mathrm{Co}_{1} \mathrm{In}_{8}$ and has a superconducting transition temperature ${ }^{9} T_{c} \sim 1 K$. On the right is the 115 structure which consists of alternating $\mathrm{CeIn}_{3}$ and $\mathrm{CoIn}_{2}$ layers giving $\left(\mathrm{CeIn}_{3}\right)\left(\mathrm{CoIn}_{2}\right)=\mathrm{CeCoIn}_{5}\left(T_{c} \sim 2.3 \mathrm{~K}\right)^{10}$. In addition, there are materials ${ }^{11}, 12$ in 


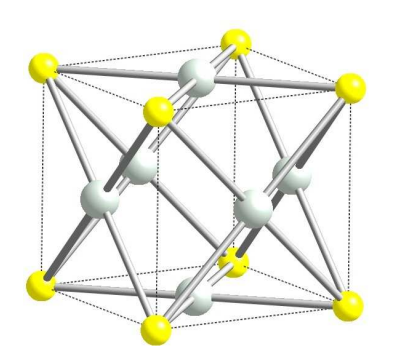

$\mathrm{Celn}_{3}$

\section{$\mathrm{Ce} \ln \mathrm{Co}$}

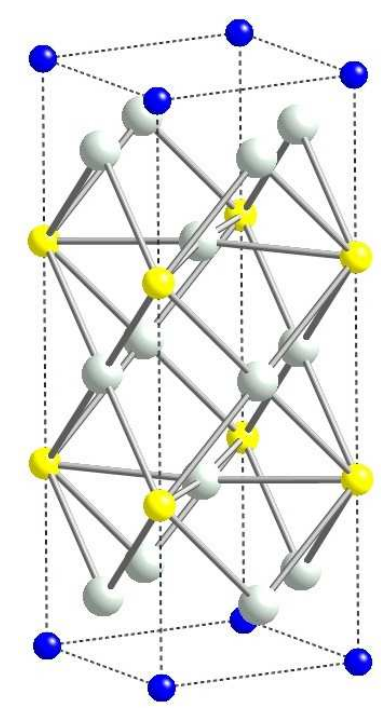

$\mathrm{Ce}_{2} \mathrm{Coln}_{8}$

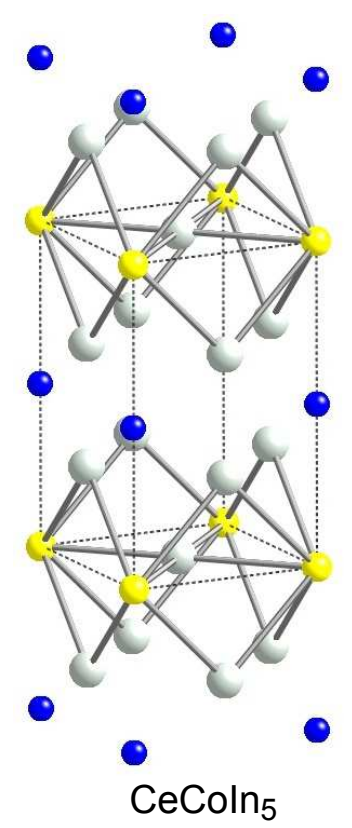

FIG. 1. Some members of the Ce family of heavy fermion superconductors. The key structural element is the quasi-two-dimensional layer of $\mathrm{Ce}^{3+}$ ions which sit at the center of a tetragon formed by 12 near neighbor $\mathrm{In}^{-}$anions. $\left(T_{c} \sim 0.2 \mathrm{~K} \mathrm{CeIn}_{3} \stackrel{8}{ }, 1.0 \mathrm{~K} \mathrm{Ce}_{2} \mathrm{CoIn}_{8} \stackrel{9}{ }, 2.3 \mathrm{~K} \mathrm{CeCoIn}_{5} \underline{10}\right)$

which Co is replaced by $\mathrm{Rh}$ or Ir, or Cd is substituted for In. The heavy-fermion actinide $\mathrm{PuMGa}_{5}$ materials have a similar structure to the $115 \mathrm{CeCoIn}_{5}$ with $\mathrm{Pu}$ replacing $\mathrm{Ce}$ and $\mathrm{Ga}$ replacing In. In this case one has $\mathrm{PuCoGa}_{5}$ with a superconducting transition temperature ${ }^{13}$ $T_{c}=18.5 \mathrm{~K}, \mathrm{PuRhGa}_{5}$ with $T_{c}=8.7 \mathrm{~K}^{14}$ as well as mixtures such as $\mathrm{Pu}\left(\mathrm{Co}_{1-x} \mathrm{Rh}_{x}\right) \mathrm{Ga}_{5}$. Recently it has been reported $\frac{15}{5}$ that $\mathrm{PuCoIn}_{5}$ becomes superconducting with $T_{c}=2.5 \mathrm{~K}$.

For the cuprates there are the well-known $\mathrm{Hg}, \mathrm{Tl}$ and $\mathrm{Bi}$ families with different numbers of $\mathrm{CuO}_{2}$ layers. The one, two and three layer members of the $\mathrm{Hg}$ family are shown in Fig. 2 , In this case the naming scheme involves four numbers. For example, for the three $\mathrm{CuO}_{2}$ layer $\mathrm{Hg} 1223$ compound $^{16,17}$ with $T_{c} \sim 135 \mathrm{~K}$ shown on the right, the first index denotes the number of $\mathrm{HgO}$ planes, the second the number of spacing $\mathrm{BaO}$ layers, the third is the number of separating $\mathrm{Ca}$ atom layers and the final the number of $\mathrm{CuO}_{2}$ layers. Thus one has the $(\mathrm{HgO})_{1}(\mathrm{BaO})_{2}(\mathrm{Ca})_{2}\left(\mathrm{CuO}_{2}\right)_{3}=\mathrm{HgBa}_{2} \mathrm{Ca}_{2} \mathrm{Cu}_{3} \mathrm{O}_{9}$ "1223" three layer material on the right and the $(\mathrm{HgO})_{1}(\mathrm{BaO})_{2}\left(\mathrm{CuO}_{2}\right)_{1}=\mathrm{HgBa}_{2} \mathrm{CuO}_{5}$ "1201" structure $\frac{18}{}$ with $T_{c} \sim 94 \mathrm{~K}^{16}$ on the left. Some of the $\mathrm{O}$ sites in the $\mathrm{Hg}$ layer are only partially occupied giving the usual chemical formulae $\mathrm{HgBa}_{2} \mathrm{CuO}_{4+\delta}$ and $\mathrm{HgBa}_{2} \mathrm{Ca}_{2} \mathrm{Cu}_{3} \mathrm{O}_{8+\delta}$. $\mathrm{A} \mathrm{Cu}$ in the $\mathrm{CuO}_{2}$ layer of the 


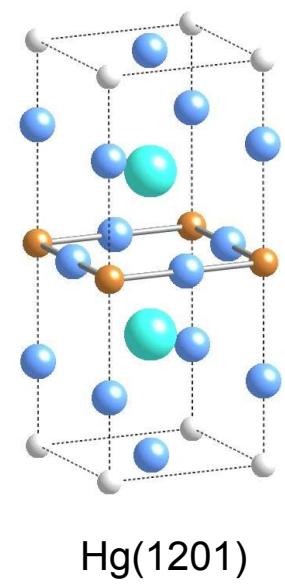

$\mathrm{Cu} \odot \mathrm{O} \mathrm{Hg} \subset \mathrm{BaOCa}$

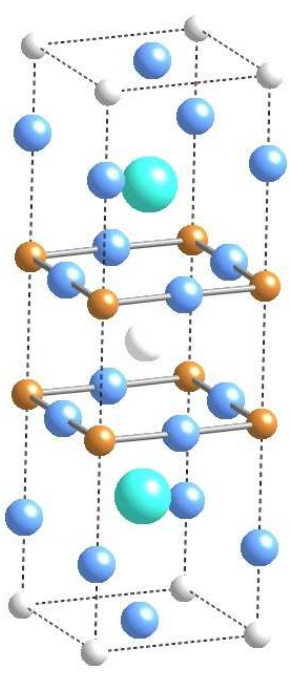

$\mathrm{Hg}(1212)$

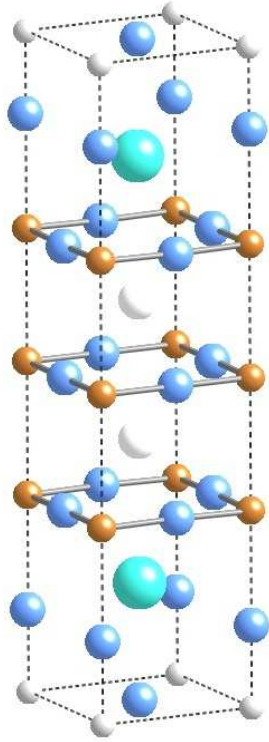

$\mathrm{Hg}(1223)$

FIG. 2. The key element of the Hg-cuprate superconductors is the $\mathrm{CuO}_{2}$ layer. The 1201 structure on the left has apical O's above and below the $\mathrm{Cu}$ sites while the inner $\mathrm{CuO}_{2}$ layer of the 1223 structure on the right has no apex oxygens (optimally doped $T_{c} \sim 94 \mathrm{~K} \mathrm{Hg}(1201), 127 \mathrm{~K} \mathrm{Hg}(1212)$, $\left.135 \mathrm{~K} \mathrm{Hg}(1223)^{\underline{16}}\right)$.

single layer 1201 material has two apical O, while a $\mathrm{Cu}$ in the middle layer of the 1223 material has none. There are also the so-called 214 families such as $\mathrm{La}_{2} \mathrm{CuO}_{4}$ which can be hole doped $\mathrm{La}_{2-x} \mathrm{M}_{x} \mathrm{CuO}_{4}$ with $\mathrm{M}=\mathrm{Sr}$ or $\mathrm{Ba}$ and $\mathrm{Nd}_{2-x} \mathrm{CuO}_{4}$ which can be electron doped $\mathrm{Nd}_{2-x} \mathrm{Ce}_{x} \mathrm{CuO}_{4}$. These latter electron doped cuprates have structures in which the apical $\mathrm{O}$ is absent. (Fig. 3) There are also the so-called infinite layer electron doped cuprates $^{21}$ in which the $\mathrm{CuO}_{2}$ planes are separated by $\mathrm{Sr}_{1-x} \operatorname{Ln}_{x}$ layers with Ln a lanthanide such as La, Sm or Nd.

Figure 4 shows some examples of the recently discovered 22,23 Fe-superconducting families which are built up from $\mathrm{Fe} /$ pnictide or chalcogen layers. In these layers the Fe ions sit on a planar two-dimensional square lattice and the pnictide or chalcogen sit at the centers of the squares, alternatively above or below the plane formed by the Fe ions. Again these layers can be stacked in a variety of ways leading to the LaOFeAs, $\mathrm{Ba}(\mathrm{FeAs})_{2}$ and FeSe structures illustrated in Fig. 4. These are called the (1111), (122) and (11) Fe-based materials, respectively. The alternating arrangement of the pnictides or chalcogens leads to a doubling of the unit 


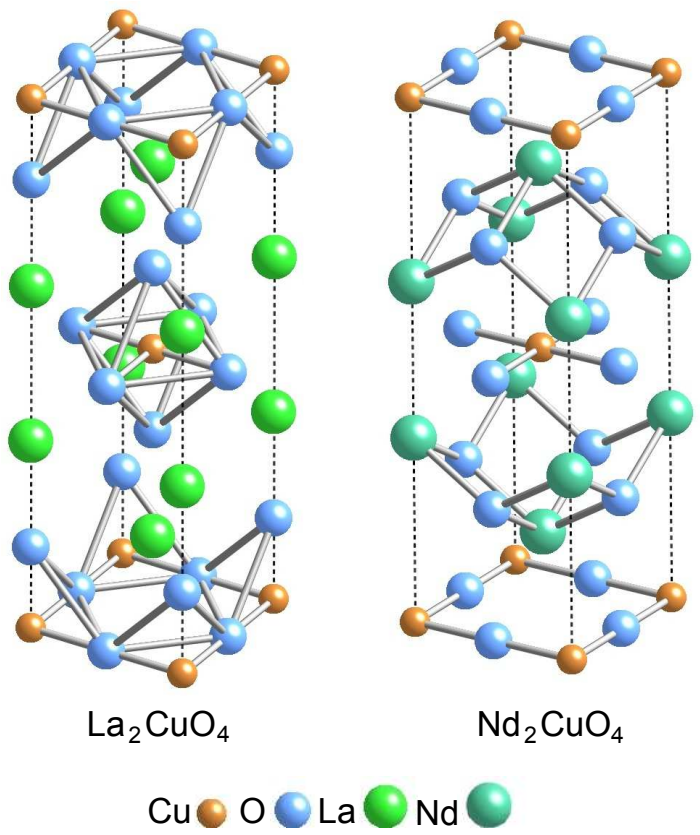

FIG. 3. The 214 cuprate structures $\mathrm{La}_{2} \mathrm{CuO}_{4}$ and $\mathrm{Nd}_{2} \mathrm{CuO}_{4}$. The former can be hole doped and the latter structure which is missing the apex oxygens can be electron doped. $\left(T_{c} \sim 38 \mathrm{~K}\right.$ $\left.\mathrm{La}_{1.85} \mathrm{Sr}_{0.15} \mathrm{CuO}_{4}{ }^{19}, 25 \mathrm{~K} \mathrm{Nd}_{1.85} \mathrm{Ce}_{0.15} \mathrm{CuO}_{4}{ }^{20}\right)$
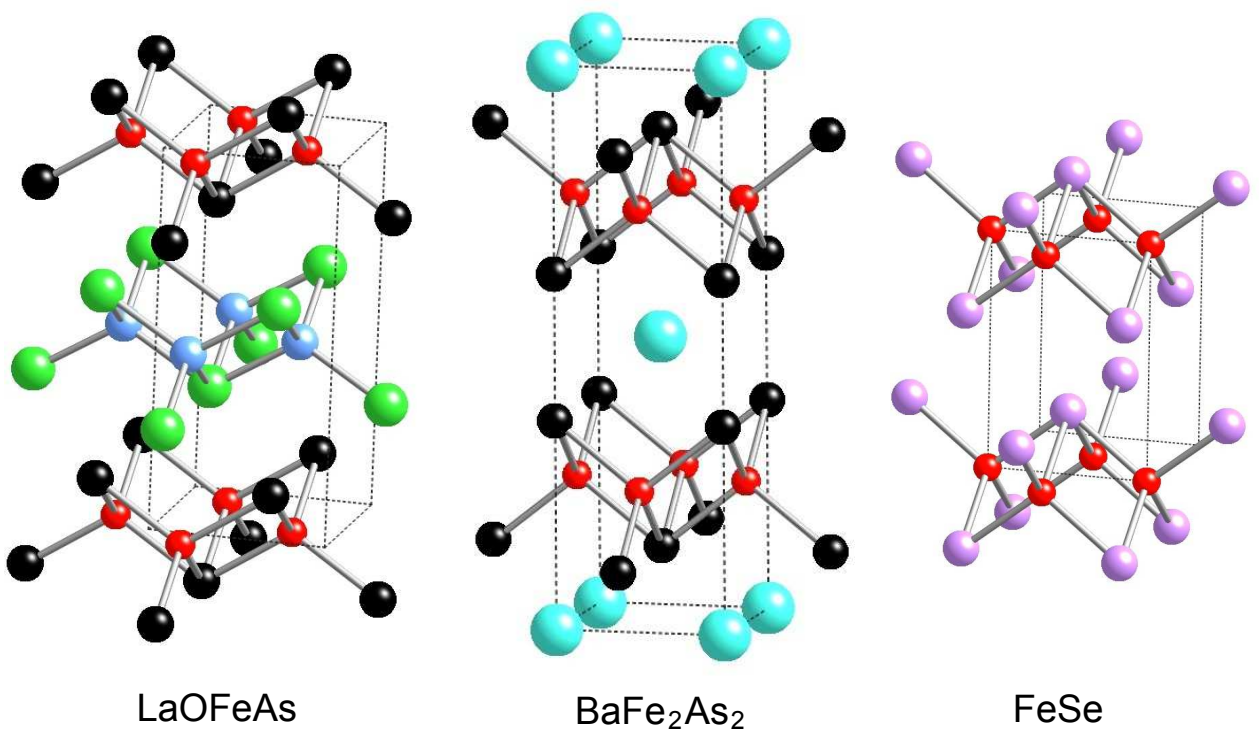

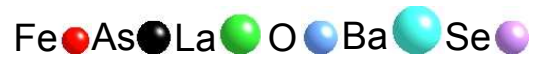

FIG. 4. Examples of the Fe-based superconductors. Here the key element is the Fe-pnictide or chalcogen layer. $\left(T_{c} \sim 26 \mathrm{~K} \mathrm{La}\left(\mathrm{O}_{0.92} \mathrm{~F}_{0.08}\right) \mathrm{FeAs}^{24}, 22 \mathrm{~K} \mathrm{Ba}\left(\mathrm{Fe}_{0.92} \mathrm{Co}_{0.08}\right)_{2} \mathrm{As}_{2} \stackrel{25}{ }, 38 \mathrm{~K}\right.$ $\left(\mathrm{Ba}_{0.6} \mathrm{~K}_{0.4}\right) \mathrm{Fe}_{2} \mathrm{As}_{2}{ }^{26}, 13.6 \mathrm{~K}$ to $\left.37 \mathrm{~K}(4.5 \mathrm{GPa}) \mathrm{FeSe}^{27}\right)$ 
cell compared with the square Fe lattice. In LaOFeAs, the Fe is tetrahedrally coordinated with four As forming square pyramids. The LaO layer has the same type of structure but with the $\mathrm{O}$ forming the square planar array. There are many equiatomic quaternary pnictide oxides of this type ${ }^{28}$. The phosphorus version of this material ${ }^{22}$ LaOFeP has a superconducting transition of $6 \mathrm{~K}$. When the As version is electron doped by replacing some of the $\mathrm{O}$ with $\mathrm{F}$ giving $\mathrm{LaO}_{1-x} \mathrm{~F}_{x} \mathrm{FeAs}$, it can become superconducting with a $T_{c}=26 \mathrm{~K}^{23}$ and replacing La with $\mathrm{Sm}$ has given $T_{c}=55 \mathrm{~K}^{29}$. In the $\mathrm{BaFe}_{2} \mathrm{As}_{2}$ (122) compound, the $\mathrm{Fe}_{2} \mathrm{As}_{2}$ layers are separated by $\mathrm{Ba}^{2+}$ ions. In this case the system can be hole doped ${ }^{30} \mathrm{Ba}_{1-x} \mathrm{~K}_{x} \mathrm{Fe}_{2} \mathrm{As}_{2}$ with an optimal $T_{c} \sim 38 \mathrm{~K}$ or electron doped ${ }^{31} \mathrm{Ba}\left(\mathrm{Fe}_{1-x} \mathrm{Co}_{x}\right)_{2} \mathrm{As}_{2}$ with $T_{c} \sim 22 \mathrm{~K}$. The third $\mathrm{Fe}(\mathrm{Se}, \mathrm{Te})$ family shown on the right hand side of Fig. 4 is essentially the infinite layer member of the family and has a $T_{c} \sim 13.6 \mathrm{~K}-37 \mathrm{~K}$ depending upon the Se/Te composition and the pressure 27,32 .

The active layers of these $\mathrm{Ce}, \mathrm{Cu}$ and $\mathrm{Fe}$ families are illustrated in Fig. 5. For the

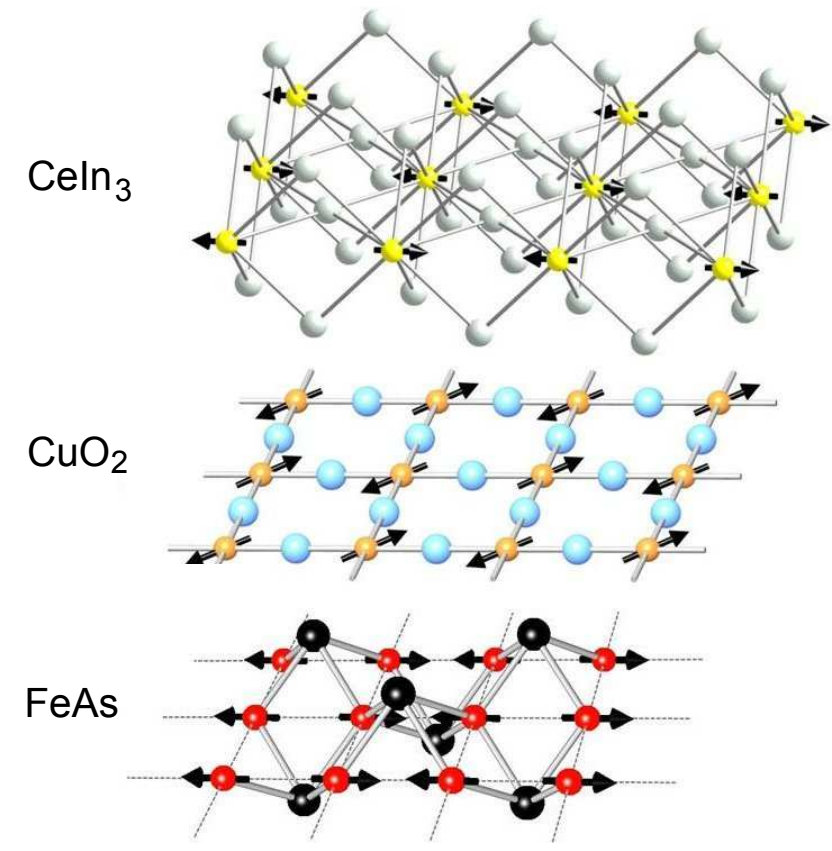

FIG. 5. The active layers of the $\mathrm{Ce}, \mathrm{Cu}$ and Fe families. The antiferromagnetic spin order of the undoped groundstates are shown.

actinide $\mathrm{Pu}$ family, the active layer is similar to the Ce layer with $\mathrm{Pu}$ replacing $\mathrm{Ce}$ and $\mathrm{Ga}$ replacing In or as recently found for the $\mathrm{PuCoIn}_{5} 115$ compound, one can simply replace Ce with $\mathrm{Pu}$. In each case, these layers contain a square sheet of metallic $d$ or $f$ cations surrounded by ligand anions. However, the spacing of the metallic ions in these compounds 
are significantly different with the $\mathrm{Ce}^{3+}$ ions separated by approximately $4.6^{\circ} \mathrm{A}$, the $\mathrm{Cu}^{2+}$ ions by $3.8^{\circ} \mathrm{A}$ and the $\mathrm{Fe}^{2+}$ ions by $2.7^{\circ} \mathrm{A}$. The $\mathrm{Fe}^{2+}$ ions are close enough that there is a direct Fe-Fe hopping which along with the d-p hybridization through the pnictogen or chalcogen anions leads to a metallic groundstate with the possibility of itinerate striped SDW antiferromagnetism and/or superconductivity. Observations of quantum oscillations originating from the Shubnikov-de Haas effect $\underline{33-} \underline{\underline{35}}$ provide clear evidence of well defined Fermi surfaces in the parent Fe-based compounds as well as the doped materials.

In contrast to this itinerant electron behavior, the undoped cuprate materials are Mott charge-transfer antiferromagnetic insulators. In the undoped $\mathrm{CuO}_{2}$ layer, one has $\mathrm{Cu}^{2+}$ in a $(3 \mathrm{~d})^{9}$ configuration. The crystal field is such that the $d_{x^{2}-y^{2}}$ orbital has the highest energy and is half-filled. The onsite $\mathrm{Cu}$ Coulomb interaction energy is large leading to the formation of local moments. The $\mathrm{O}$ orbital mediates an exchange interaction $\underline{\underline{36}}$ between the $\mathrm{Cu}$ spins and the groundstate has long range antiferromagnetic order. In the three dimensional crystal, the interlayer exchange coupling leads to a finite Néel temperature. The undoped system is a charge-transfer insulator with a gap set by the difference in energy between the $2 \mathrm{p}$ state of the $\mathrm{O}$ and the $d_{x^{2}-y^{2}}$ state of the $\mathrm{Cu}$. In order to have metallic behavior and the possibility of superconductivity, the $\mathrm{CuO}_{2}$ planes need to be doped. The occupancy of the oxygen site in the $\mathrm{Hg}$ layer typically controls the hole doping of the $\mathrm{CuO}_{2}$ in the $\mathrm{Hg}$ cuprates while cation substitution or $\mathrm{O}$ doping excess or depletion can provide hole or electron doping for the 214 cuprates.

In the heavy fermion materials one has the largest ion separation but in this case the conduction band of the ligands gives rise to a metallic state. The 14-fold degenerate $f$ electronic states of the $(4 f)^{1}$ configuration of $\mathrm{Ce}^{3+}$ are split by a large spin-orbit coupling into a low lying $j=5 / 2$ sextet and a higher energy $j=7 / 2$ octet. The one electron states of the $j=5 / 2$ sextet are further split by the crystalline electric field of the In ligand anions into three sets of Kramer's doublets ${ }^{37}$. Then, depending upon the strength of the hybridization, these states are localized or delocalized. For example, CeRhIn ${ }_{5}$ has an antiferromagnetic groundstate in which the $4 f$-electron of Ce is localized with a magnetic moment only slightly reduced from its full atomic value ${ }^{12}$. The system is metallic due to the conduction band associated with the ligands. Under sufficient pressure, $1.7 \mathrm{GPa}$, the $4 f$-electron takes on some itinerant character and the system becomes superconducting 38 . In $\mathrm{CeCoIn}_{5}$ and $\mathrm{CeIrIn}_{5}$, at low temperatures the $4 f$ electron are delocalized through their 
coupling with the ligand conduction band and these systems become superconducting at atmospheric pressure ${ }^{10} \underline{12}$. Replacing a small amount of In with a few percent of Cd leads to a metallic antiferromagnetic state ${ }^{39,40}$. The two-dimensional character of the Ce ion layers lead to nearly cylindrical Fermi surfaces which are seen in de Haas and van Alphen measurements. The cyclotron masses are large consistent with the fact that the $4 f$ electrons make a contribution to the Fermi surface states 41 .

\section{B. Phase diagrams}

These materials exhibit a range of different phases. There are tetragonal and orthorhombic lattice phases, nematic electronic phases, charge density wave and striped magnetic phases, charge-transfer antiferromagnetic Mott insulating as well as metallic spin density wave phases, and of course superconductivity. Via temperature, doping, chemical or hydrostatic pressure, or the application of a magnetic field one can change the phase of these materials. However, the feature that is striking in the phase diagrams for all of these materials is the proximity of the antiferromagnetic or spin density wave and superconducting phases. These phases may in some cases coexist or alternatively there may be a first order transition from the AF state to the superconducting state. Then as noted by Emery et al. $\stackrel{42}{\text {, }}$ Coulomb frustrated phase separation can lead to a mesoscopic phase in which a lightly doped locally AF and a more heavily hole doped region are in close contact. It has been suggested that this type of inhomogeneity may in fact lead to an optimal superconducting transition temperature ${ }^{43}$.

Examples of phase diagrams for the heavy fermion, cuprate and Fe-based materials are shown in Figs. 6, 8 . The phase diagram for the 115 heavy fermion system ${ }^{39} \mathrm{CeCo}\left(\operatorname{In}_{1-\mathrm{x}} \mathrm{Cd}_{\mathrm{x}}\right)_{5}$ is shown in Fig. [6a. For $x=0, \mathrm{CeCoIn}_{5}$ becomes superconducting at temperatures below approximately $2.3 \mathrm{~K}$. Then as the $\mathrm{Cd}$ concentration increases, one enters a region where the system first becomes antiferromagnetic and then below the superconducting $T_{c}$ there is a coexistence regime. Finally, for Cd concentration $x \gtrsim 0.15$, superconductivity is absent and the Néel temperature $T_{N}$ continues to increase. A similar phase diagram for the case in which Co is replaced by Ir is shown in Fig. 6b. In this case, while the Néel temperatures are comparable to those of the Co material, the superconducting $T_{c}$ is significantly smaller.

Figure 7 shows the phase diagrams of $\mathrm{La}_{2-x} \mathrm{Sr}_{x} \mathrm{CuO}_{4}$ and $\mathrm{Nd}_{2-x} \mathrm{Ce}_{x} \mathrm{CuO}_{4}{ }_{44}$. Undoped 

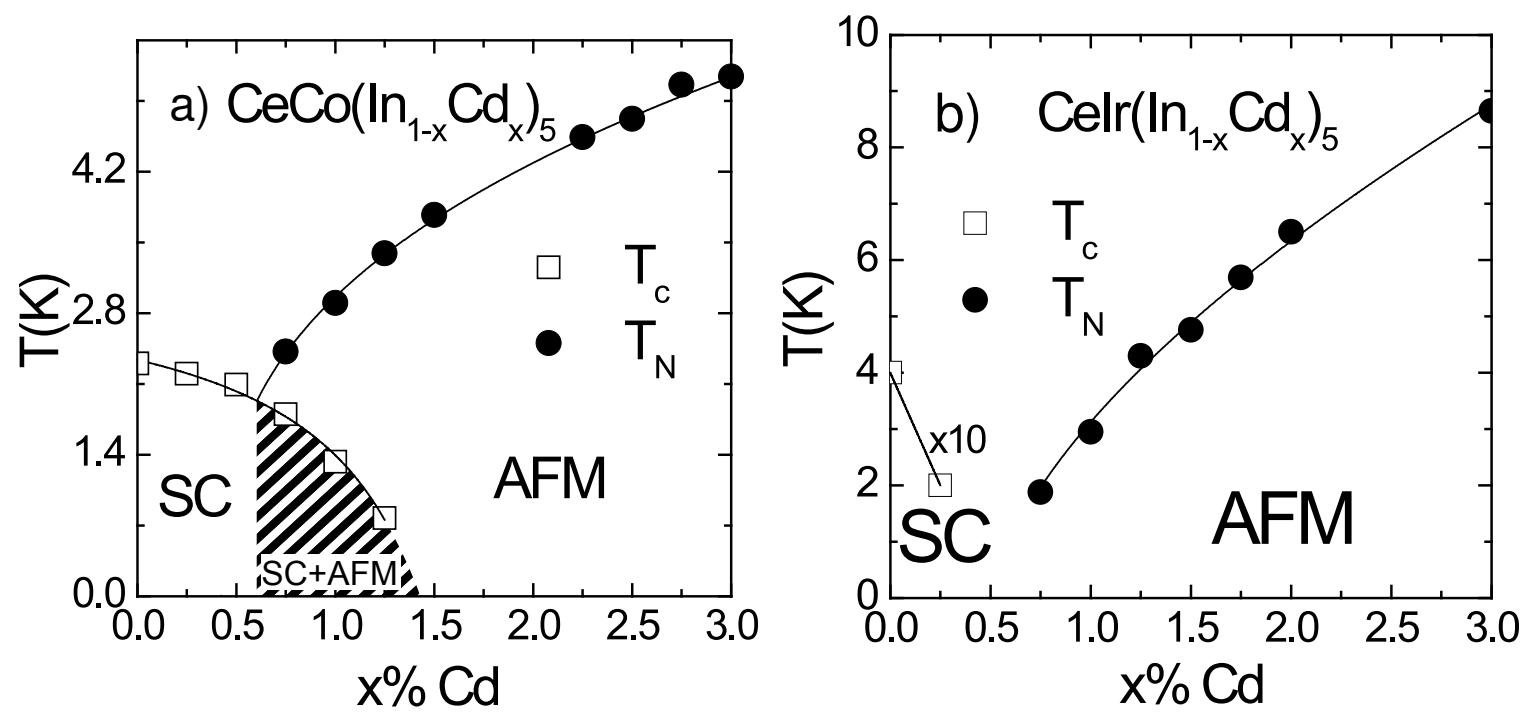

FIG. 6. Phase diagrams for two heavy fermion Ce-115 systems: (a) $\mathrm{CeCo}\left(\operatorname{In}_{1-x} \mathrm{Cd}_{x}\right)_{5}$ (after Nicklas et al. $\underline{40})$ and (b) $\operatorname{CeIr}\left(\operatorname{In}_{1-x} \mathrm{Cd}_{x}\right)_{5}$ (after Pham et al. $\left.\underline{\underline{39}}\right)$. Note that $T_{c}$ is multiplied by a factor of 10 for $\operatorname{CeIr}\left(\operatorname{In}_{1-x} \mathrm{Cd}_{x}\right)_{5}$. In both cases one sees the close proximity of superconductivity and antiferromagnetism. For the Co compound there is a region of coexistence.

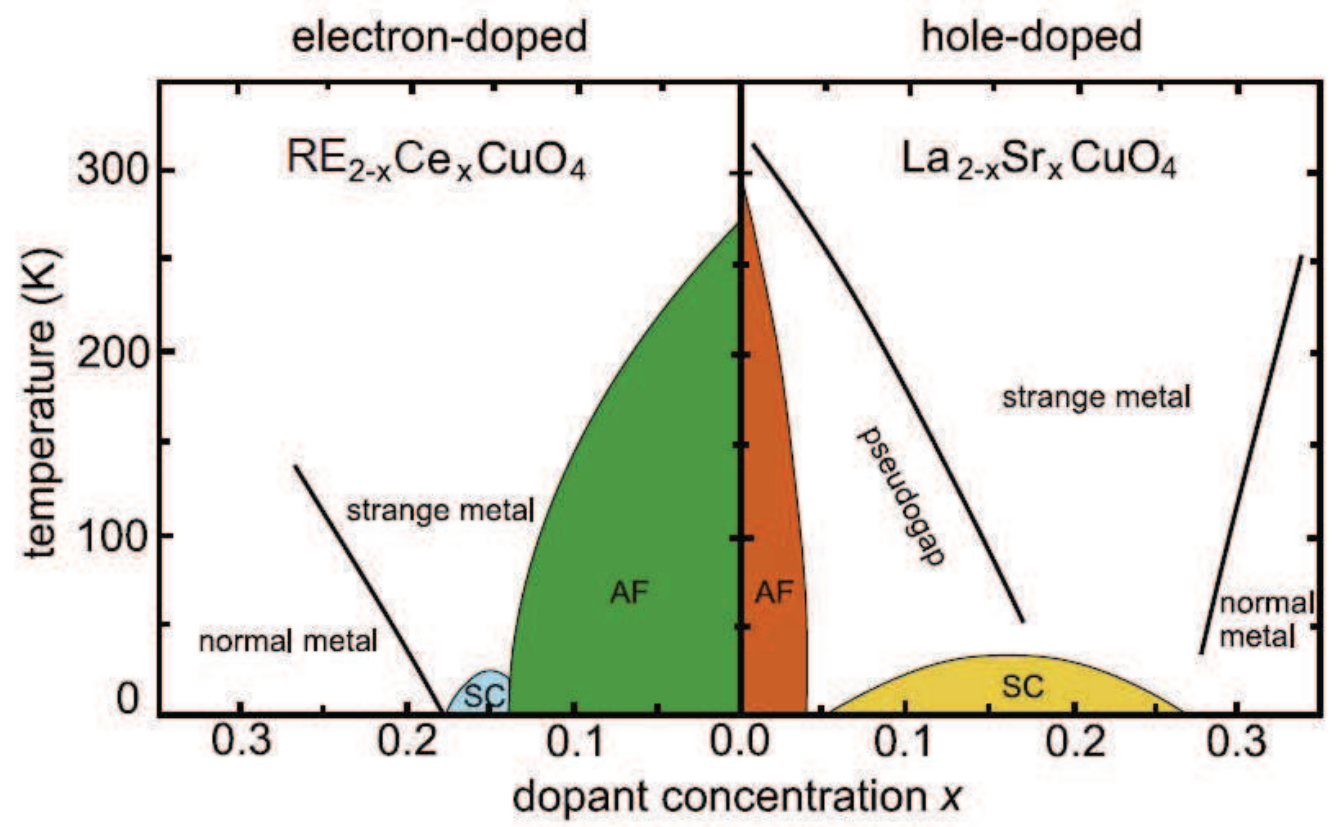

FIG. 7. Schematic phase diagrams for hole doped $\mathrm{La}_{2-x} \mathrm{Sr}_{x} \mathrm{CuO}_{4}$ and electron-doped $\mathrm{RE}_{2-x} \mathrm{Ce}_{x} \mathrm{CuO}_{4}(\mathrm{RE}=\mathrm{La}, \mathrm{Pr}, \mathrm{Nd})$ cuprates (after R.L. Greene and Kui Jin). In the electrondoped case, the AF region extends to the superconducting region, while in the hole-doped case a pseudogap region intervenes. 
$\mathrm{La}_{2} \mathrm{CuO}_{4}$ and $\mathrm{Nd}_{2} \mathrm{CuO}_{4}$ are charge-transfer insulators which undergo antiferromagnetic Néel transitions as the temperature drops below 300K. Replacing a small amount of La with $\mathrm{Sr}$ leads to a hole doping of the $\mathrm{CuO}_{2}$ layer, while replacing $\mathrm{Nd}$ with Ce leads to an electron doped $\mathrm{CuO}_{2}$ layer. As the hole doping $x$ increases, the Néel temperature is suppressed and at low temperatures the system passes through a spin glass phase in which local charge and spin ordered regions may be pinned. In the hole doped case, the doping for optimal superconductivity is well separated from the onset of antiferromagnetism. The antiferromagnetic order extends much further out for the electron doped system and appears adjacent to the superconducting phase.

The phase diagram for one of the Fe-based superconductors ${ }^{45} \mathrm{Ba}\left(\mathrm{Fe}_{1-x} \mathrm{Co}_{x}\right) \mathrm{As}_{2}$ is shown in Fig. 8, The parent compound $\mathrm{BaFe}_{2} \mathrm{As}_{2}$ is metallic and undergoes a structural tetragonal

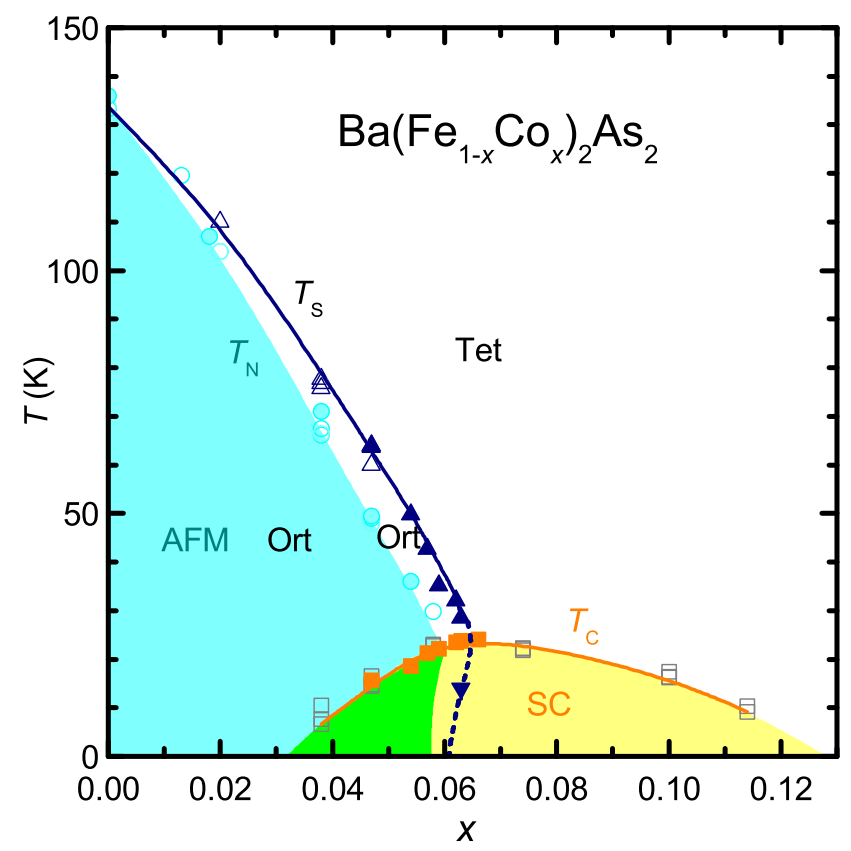

FIG. 8. The phase diagram for $\mathrm{Ba}\left(\mathrm{Fe}_{1-x} \mathrm{Co}_{x}\right) \mathrm{As}_{2}$ (after Fernandes et al. $\underline{45}$ ). There appears a coexistence region similar to $\mathrm{CeCo}\left(\operatorname{In}_{1-x} \mathrm{Cd}_{x}\right)_{5}$ shown in Fig. 6.

to orthorhombic transition and at the same temperature an antiferromagnetic SDW transition. In the SDW phase the moments are oriented antiferromagnetically along the longer $a_{0}$ axis of the orthorhombic $2 \mathrm{Fe} /$ cell and ferromagnetically along the $b_{0}$ axis giving a stripe-like structure. As Co is added, the system is electron doped and the structural and SDW transitions are suppressed. The structural transition is found to occur at temperatures slightly 
above the SDW transition. For dopings $x \gtrsim 0.07$, the structural and SDW transitions are completely suppressed and the system goes into a superconducting state below $T_{c}$. However, for a range of smaller dopings $0.03 \lesssim x \lesssim 0.06$ the system enters a region in which there is microscopic coexistence of superconductivity, SDW and orthorhombic order. As will be discussed, evidence for this is seen in the temperature dependence of the SDW Bragg peak intensity and the orthorhombic distortion. It is also possible to hole dope this compound ${ }^{30}$ by substituting $\mathrm{K}$ for $\mathrm{Ba}, \mathrm{Ba}_{1-x} \mathrm{~K}_{x} \mathrm{Fe}_{2} \mathrm{As}_{2}$. Here again, as $x$ increases the structural and SDW transition are suppressed and superconductivity onsets $\underline{46}$.

\section{Coexistence and interplay of antiferromagnetism and superconductivity}

NMR as well as neutron scattering measurements have provided evidence that the observed coexistence regions in some systems represent microscopic coexistence in which the same electrons are involved with both the superconductivity and the antiferromagnetism. For example, elastic neutron scattering measurements ${ }^{39}$ on $\mathrm{CeCo}\left(\operatorname{In}_{0.9} \mathrm{Cd}_{0.1}\right)_{5}$ find the integrated magnetic intensity at the antiferromagnetic wave vector $Q_{\mathrm{AF}}$ versus temperature shown in Fig. 9a. This intensity is a measure of the square of the ordered magnetic moment and onsets at the Néel temperature $T_{N}$. As seen in Fig. 9 $\mathrm{a}, M^{2}(T)$ initially increases as $T$ decreases below $T_{N}$, but then as $T$ drops below the superconducting transition temperature $T_{c}$, it saturates. Similar data for $\mathrm{Ba}\left(\mathrm{Fe}_{1-\mathrm{x}} \mathrm{Co}_{\mathrm{x}}\right) \mathrm{As}_{2}$ at three different dopings are shown in Fig. 9b. In this case, below $T_{c}$ the ordered moment is reduced as the superconducting order increases. Both these examples reflect the competition of superconductivity and antiferromagnetism $\underline{45}, \underline{47}$. This competition is also believed to be responsible for the anomalous suppression of the orthorhombic distortion in $\mathrm{Ba}\left(\mathrm{Fe}_{1-x} \mathrm{Co}_{x}\right) \mathrm{As}_{2}$ as the temperature decreases below $T_{c} \underline{\underline{48}}$. Evidence for atomic scale coexistence of superconductivity and antiferromagnetism for $\mathrm{Ba}\left(\mathrm{Fe}_{1-x} \mathrm{Co}_{x}\right)_{2} \mathrm{As}_{2}$ with $x=0.06$ was reported by LaPlace et al. $\underline{\underline{49}}$. Here volume susceptibility measurements showed a superconducting fraction greater than $95 \%$. Then measurements of the homogeneous broadening of the ${ }^{75}$ As NMR spectrum showed that frozen moments remained on all of the Fe atoms for $T$ less than $T_{c}$ while at the same time, the spin-lattice relaxation rate $T_{1}^{-1}$ of ${ }^{75}$ As showed that the Fe electrons also exhibited superconductivity. Since the As nuclei are coupled to only the four near neighbor Fe sites, this experiment provided evidence of homogeneous coexistence on a unit cell scale. 

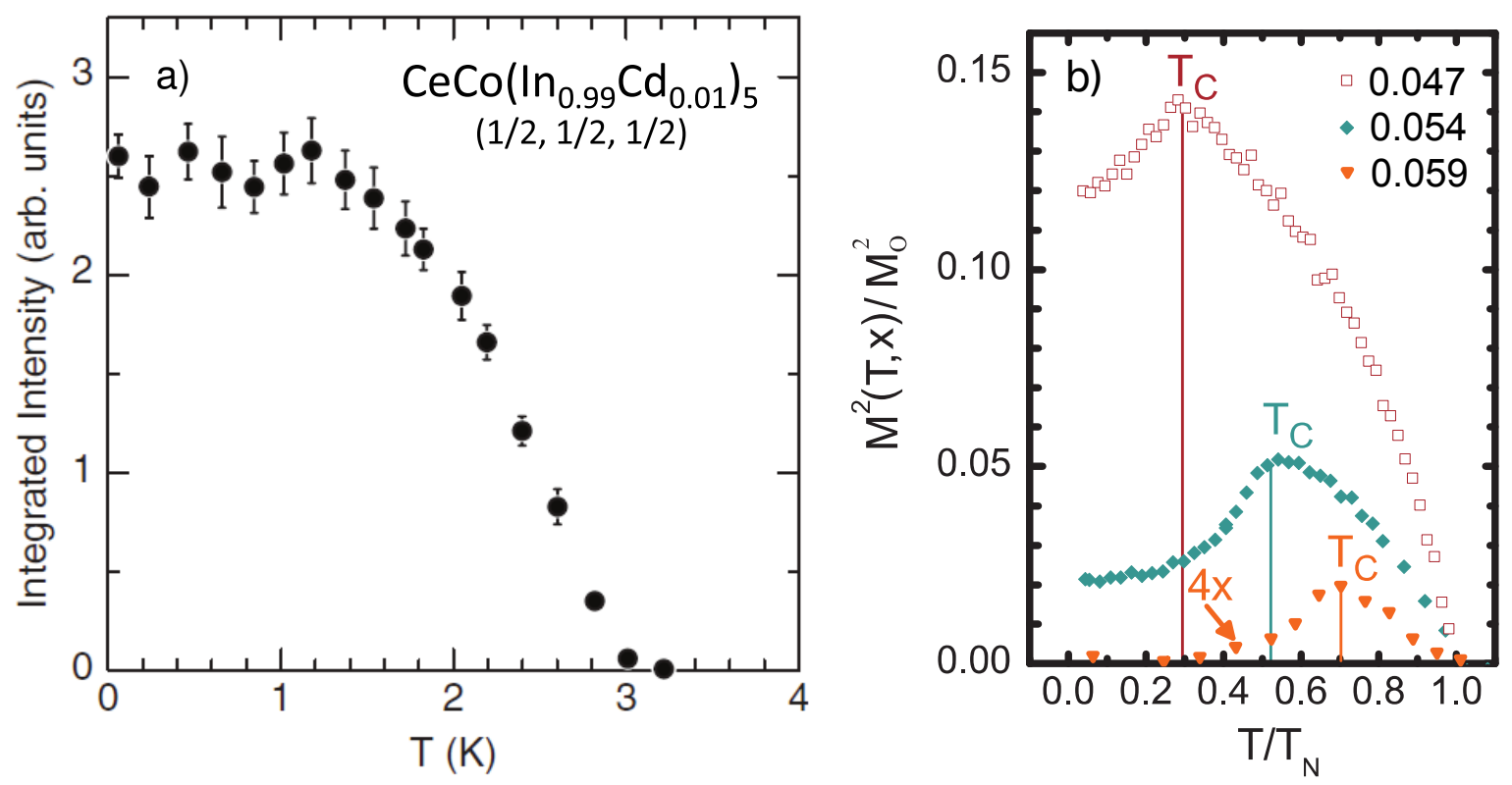

FIG. 9. The interplay of antiferromagnetism and superconductivity is seen in the temperature dependence of the Bragg scattering. (a) The integrated Bragg scattering intensity for the 115 heavy fermion superconductor $\mathrm{CeCo}\left(\operatorname{In}_{0.99} \mathrm{Cd}_{0.01}\right)_{5}$ at $Q_{\mathrm{AF}}$ versus temperature (after Nicklas et al. $\stackrel{40}{ })$. (b) The integrated Bragg scattering intensity for $\mathrm{Ba}\left(\mathrm{Fe}_{1-x} \mathrm{Co}_{x}\right) \mathrm{As}_{2}$ at $Q_{\mathrm{SDW}}$ versus the temperature for various values of $x$ (after Fernandes et al. 45 ). In both cases, the strength of the Bragg scattering from the magnetic order is clearly altered by the onset of the superconductivity.

In addition to the ordered antiferromagnetic (Néel) phase, there are a variety of incommensurate spin density wave striped phases that compete and interact with the superconducting phase. Evidence of this is seen in neutron scattering experiments on $\mathrm{La}_{2-x} \mathrm{Sr}_{x} \mathrm{CuO}_{4}$ which reveal a strong enhancement of spin-stripe order at low energies produced by modest magnetic fields 50,51 . This behavior has been modeled by Landau-Ginzburg theories in which the incommensurate antiferromagnetic order is coupled to the $d$-wave superconducting order 52,53 . This mutual coupling of SDW and $d$-wave scattering processes has also been found in renormalization group calculations ${ }^{54}-\underline{57}$.

A particularly striking example of the coexistence and interplay of antiferromagnetism and $d$-wave superconductivity is seen in $\mathrm{La}_{2-x} \mathrm{Ba}_{x} \mathrm{CuO}_{4}$ near a doping $x \sim 1 / 8 \underline{58,59}$. Here a combination of tunneling and photoemission measurements along with transport studies provide evidence that two-dimensional $d$-wave superconducting correlations coexist with $\pi$-phase shifted antiferromagnetic stripes at temperatures below $40 \mathrm{~K}$. The observation that 
macroscopic 2D superconductivity persists at temperatures well above the 3D transition temperature suggests that the pairing correlations form a pair density wave with a wavevector which is the same as that of the spin-density wave $\underline{60,61}$. That is, the amplitude of the $d$-wave superconducting order parameter is enhanced in the hole-rich regions of the striped system and the phase of the adjacent superconducting stripes are opposite in sign (antiphase). In this case, the structurally driven orthogonal orientation of the stripes in neighboring planes leads to a frustration of the Josephson coupling between planes allowing for the possibility of a Berezinskii-Kosterlitz-Thouless transition in the 3D crystal.

The interplay between the antiferromagnetic spin fluctuations and the superconducting pairs is also seen in the change in the exchange energy $\Delta E_{\text {ex }}$ between the superconducting and normal states ${ }^{62}$. For a material with a near neighbor exchange coupling $J$, the change in exchange energy $\Delta E_{\text {ex }}(T)$ is given by

$$
\Delta E_{\mathrm{ex}}(T)=2 J\left(\left\langle\mathbf{S}_{i+x} \cdot \mathbf{S}_{i}\right\rangle_{N}-\left\langle\mathbf{S}_{i+x} \cdot \mathbf{S}_{i}\right\rangle_{S}\right)
$$

with

$$
\left\langle\mathbf{S}_{i+x} \cdot \mathbf{S}_{i}\right\rangle_{S(N)}=\frac{1}{g^{2} \mu_{\beta}^{2}} \int_{0}^{\infty} \frac{d \omega}{\pi}(n(\omega)+1)\left\langle\cos \left(q_{x} a\right) \chi_{S(N)}^{\prime \prime}(q, \omega)\right\rangle_{B Z}
$$

Here $n(\omega)$ is the usual Bose factor, the momentum $q$ is summed over the Brillouin zone and $\chi_{S(N)}^{\prime \prime}(q, \omega)$ is the imaginary part of the wavevector and frequency dependent spin susceptibility in the superconducting $(S)$ and normal $(N)$ phases respectively, measured at temperature T. Additional next-near-neighbor exchange terms appropriate to a given material can be added to Eq. (1). In initial studies of $\mathrm{YBa}_{2} \mathrm{Cu}_{3} \mathrm{O}_{6.95}\left(T_{c}=92.5 \mathrm{~K}\right)$, a low temperature value of $\Delta E_{\text {ex }}$ was estimated from measurements of $\chi_{s}^{\prime \prime}(q, \omega)$ at $T=15 \mathrm{~K}$ and $\chi_{N}^{\prime \prime}(q, \omega)$ taken at 100K. This estimate gave a change in the exchange energy which was approximately 15 times larger than the superconducting condensation energy ${ }^{63}$. Recent measurements of the heavy fermion superconductor $\mathrm{CeCu}_{2} \mathrm{Si}_{2}$ found a change of the exchange energy which was of order 20 times larger than its low temperature superconducting condensation energy $\underline{\underline{64}}$. In this case, the lower $T_{c} \sim 0.6 \mathrm{~K}$ of this heavy fermion systems allowed direct access at this same temperature to the putative normal state using a $2.5 T$ magnetic field. While the superconducting condensation energy $U_{c}$ arises from a cancellation between this change in the exchange energy $\Delta E_{\mathrm{ex}}$ and other electronic energies, the important point is that $\Delta E_{\mathrm{ex}}$ is large compared with $U_{c}$ so that antiferromagnetic fluctuations clearly have the strength 
to drive the superconducting pairing. In addition, we note that $\Delta E_{\mathrm{ex}} / U_{c}$ is similar in size for $\mathrm{YBa}_{2} \mathrm{Cu}_{3} \mathrm{O}_{6.95}$ and $\mathrm{CeCu}_{2} \mathrm{Si}_{2}$.

The similarities of the suppression of the Bragg scattering intensity $M^{2}$ in the coexisting antiferromagnetic and superconducting state, the magnetic field induced SDW in the superconducting state and the change of the exchange energy between the superconducting and normal paramagnetic states not only serve to establish a relationship between these different materials but in addition provide evidence that the antiferromagnetism and superconductivity in these materials are strongly coupled. Further evidence of this is also clearly seen in NMR studies of the spin-lattice relaxation time $T_{1}$ of $\mathrm{FeSe}^{65}$ and inelastic neutron scattering measurements of overdoped LSCO ${ }^{66}$. Measurements of $\left(T_{1} T\right)^{-1}$ probe the Brillouin zone average of $\operatorname{Im} \chi\left(q, \omega_{0}\right) / \omega_{0}$ weighted with the square of the hyperfine form factor. Here $\omega_{0}$ is a low frequency set by the nuclear Zeeman energy. As the pressure is increased on FeSe, $\left(T_{1} T\right)^{-1}$ and $T_{c}$ are both enhanced. Similarly, the strength of the low-energy incommensurate antiferromagnetic spin fluctuations in overdoped LSCO is observed to decrease ${ }^{66}$ as the doping increases and $T_{c}$ is reduced.

While neutron scattering measurements provide evidence of the $q-\omega$ spin-fluctuation spectral weight for the underdoped materials $\frac{63}{}$, one is of course also interested in the optimally as well as the overdoped materials. Recently 67 , resonant inelastic x-ray scattering (RIXS) experiments have provided such information over a wide energy-momentum region for $\mathrm{YBa}_{2} \mathrm{Cu}_{4} \mathrm{O}_{8}, \mathrm{YBa}_{2} \mathrm{Cu}_{3} \mathrm{O}_{6+x}$, and $\mathrm{Nd}_{1.2} \mathrm{Ba}_{1.8} \mathrm{Cu}_{3} \mathrm{O}_{6+x}$. These experiments clearly show, for a range of dopings covering underdoped, optimal as well as over-doped materials, the existence of damped, dispersive magnetic excitations, which have significant spectral weight in an appropriate spectral range to produce pairing.

There is also resistivity data which provides evidence of the strong coupling of the spinfluctuations and the quasiparticles in the regions of the phase diagram where superconductivity appears. Taillefer has emphasized the similar behavior of the temperature dependent part of the in-plane normal state resistivity of the cuprate Nd-LSCO, the organic Bechgaard salt (TMTSF) $)_{2} \mathrm{PF}_{6}$ and the Fe-pnictide $\mathrm{Ba}\left(\mathrm{Fe}_{1-x} \mathrm{Co}_{x}\right) \mathrm{As}_{2}$ shown in Fig. 10. Here the linear $T$ dependence of the resistivity of Nd-LSCO is associated with a hole doping 0.24 at which the stripe-ordered antiferromagnetic phase ends $\frac{68}{}$. Likewise, a Co concentration $\sim 0.10$ for Co-Ba122 and a pressure $\gtrsim 10 \mathrm{kbar}$ for $(\mathrm{TMTSF})_{2} \mathrm{PF}_{6}$ mark the ends of the SDW phases for these materials. As the doping (or pressure for the Bechgaard salt) is increased, the 

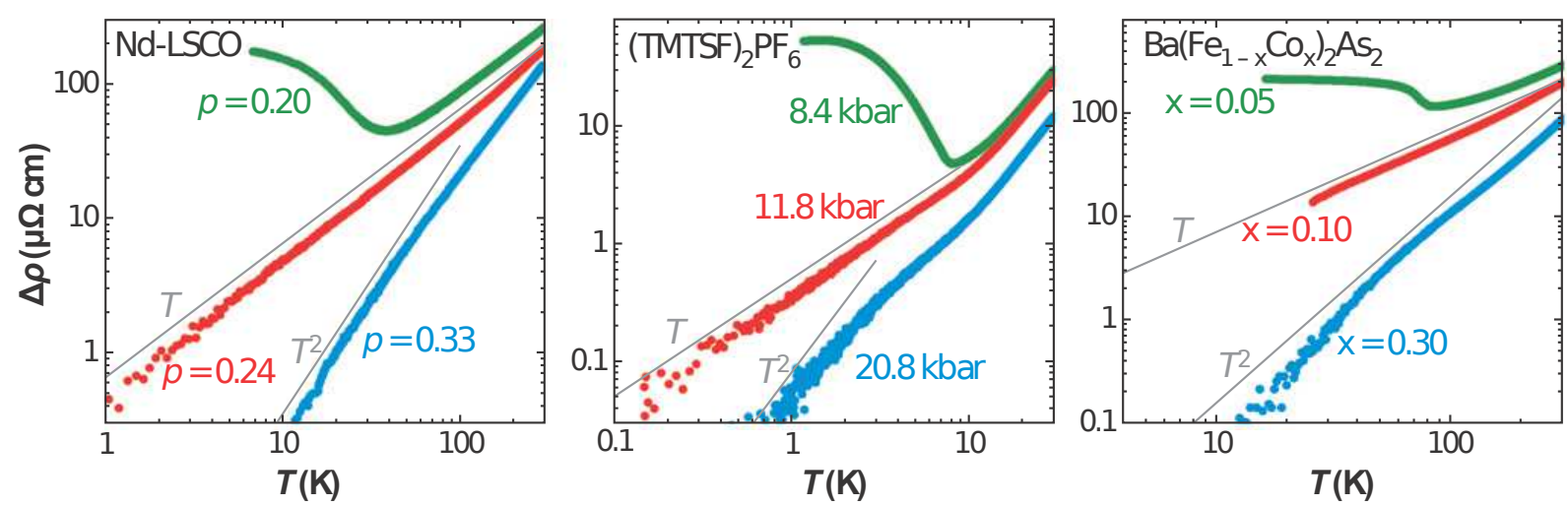

FIG. 10. The temperature dependent part $\Delta \rho(T)$ of the in-plane resistivity versus $T$ on a $\log -\log$ scale for the cuprate Nd-LSCO, the organic Bechgaard salt (TMTSF) ${ }_{2} \mathrm{PF}_{6}$ and the Fe-pnictide $\mathrm{Ba}\left(\mathrm{Fe}_{1-x} \mathrm{Co}_{x}\right)_{2} \mathrm{As}_{2}$. As a relevant tuning parameter, doping or pressure, is changed, the temperature dependence of $\Delta \rho(T)$ for all three systems pass from a $T^{2}$ dependence to an approximately linear $T$ dependence and then to an upturn associated with a Fermi surface reconstruction (after Taillefer $\left.{ }^{5}\right)$.

anomalous $T$ dependence is replaced by a Fermi-liquid $T^{2}$ dependence and the superconducting $T_{c}$ goes to zero. At low doping or under pressure, the upturn in $\Delta \rho$ shows evidence of a Fermi-surface reconstruction due to the occurrence of an ordered phase. Based on transport and NMR measurements on the (TMTSF) $)_{2} \mathrm{X}$ materials as a function of pressure, Doiron-Leyraud et al. $\underline{\underline{6}, \underline{69}}$ argue that the linear $T$ dependence of the resistivity is associated with scattering from antiferromagnetic spin fluctuations at the border of antiferromagnetic order and that this scattering is directly linked to $T_{c}$. Hartnoll et al. $\underline{70}$ have argued that a quantum critical response arises from spin-fluctuation scattering and umklapp processes as the spin density wave phase of a $2 \mathrm{D}$ metal is approached.

A similar connection between spin-fluctuation scattering of the carriers and the basal plane resistivity of $\mathrm{La}_{2-x} \mathrm{Ce}_{x} \mathrm{CuO}_{4}$ films has been reported by Jin et al. $\stackrel{71}{ }$. These authors carried out low temperature resistivity experiments as a function of doping and magnetic field. They found a correlation between the strength of the low temperature linear-in- $T$ resistivity and the superconducting $T_{c}$ as a function of doping. They noted that this electron doped cuprate provided a particularly interesting case since there is no pseudogap phase in the underdoped region of its phase diagram, leaving the spin fluctuations as the dominant link to the temperature dependence of the resistivity. 
A magnetic field-tuned quantum critical response is also seen in the heavy fermion $\mathrm{CeCoIn}_{5}$ system $^{72}$ as well as other heavy fermion materials. Of particular interest, as $\mathrm{Si}$ and Steglich $\underline{73}$ have discussed for $\mathrm{CaCu}_{2} \mathrm{Si}_{2}$ and $\mathrm{CePd}_{d} \mathrm{Si}_{2}$, are the antiferromagnetic to paramagnetic quantum critical transitions. Here, the critical degrees of freedom are the SDW fluctuations. The role of the quantum critical point and the interplay between antiferromagnetism and the resulting temperature, carrier concentration and magnetic field phase diagram have been discussed by Sachdev and Metlitski (2010). To summarize, the possible coexistence of antiferromagnetism and $d$-wave superconductivity, the change in the exchange energy upon entering the superconducting phase and the importance of spin-fluctuation scattering are characteristic of the class of materials being discussed.

\section{A neutron spin resonance}

Another important experimental observation linking these materials is the appearance of a neutron scattering spin resonance in the superconducting phase at the antiferromagnetic or spin-density-wave vector $Q$. This resonance, first observed in the cuprates ${ }^{74} \underline{-77}$ and then discovered in the heavy fermion materials ${ }^{78}$, has also recently been observed in various $\mathrm{Fe}$ superconductors $99-82$. The spin-flip inelastic scattering rate is proportional to the imaginary part of the spin susceptibility. Experimental results for $\chi^{\prime \prime}(Q, \omega)$ obtained for $\operatorname{CeCoIn}_{5}$, $\mathrm{Bi}_{2} \mathrm{Sr}_{2} \mathrm{CaCu}_{2} \mathrm{O}_{8+\delta}$ and $\mathrm{BaFe}_{1.85} \mathrm{Co}_{0.15} \mathrm{As}_{2}$ are shown in Figs. 11,13. While the energy of the resonant peak in YBCO is relatively insensitive to $T / T_{c}$, the peak in $\mathrm{Ba}\left(\mathrm{Fe}_{0.975} \mathrm{Co}_{0.125}\right)_{2} \mathrm{As}_{2}$ was found to follow the temperature dependence of the superconducting gap obtained from ARPES $83,84$.

Although the detailed behavior of the resonance requires a calculation of the spin susceptibility, the occurrence of the resonance is directly related to the BCS coherence factor that enters the neutron spin-flip scattering process. This coherence factor for flipping the spin of a quasi-particle scattered from $k$ to $k+Q$ is

$$
\frac{1}{2}\left(1-\frac{\Delta(k) \Delta(k+Q)}{E(k) E(k+Q)}\right)
$$

with $E(k)=\sqrt{\varepsilon_{k}^{2}+\Delta^{2}(k)}$ the quasi-particle energy. The occurrence of a resonance, requires that the gap changes sign between regions on the Fermi surface or surfaces separated by 


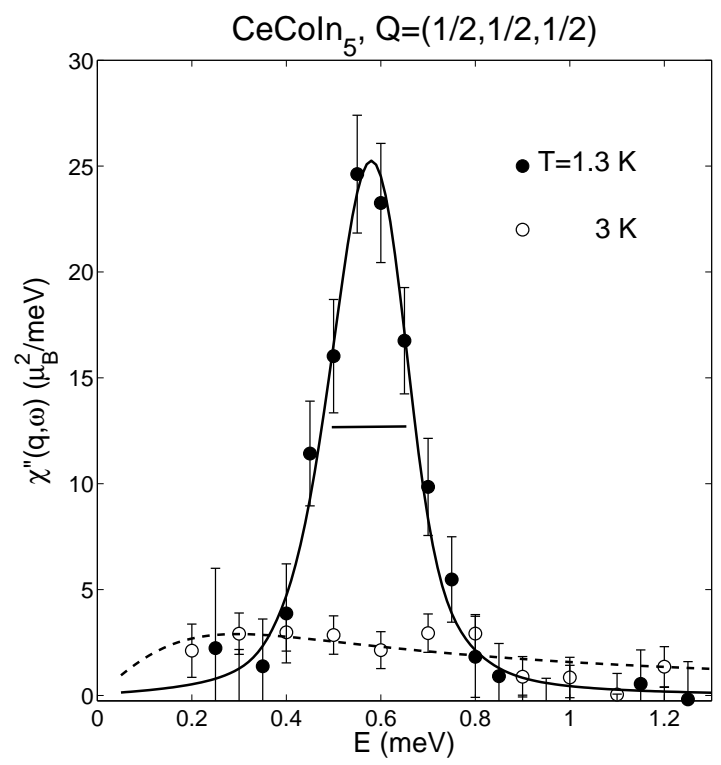

FIG. 11. The neutron scattering spin resonance in the normal (dashed) and superconducting (solid) phases observed for the 115 Ce heavy fermion material $\mathrm{CeCoIn}_{5}\left(\mathrm{~T}_{c}=2.3 \mathrm{~K}\right)$ (after Stock et al. $\left.\stackrel{78}{ }\right)$.

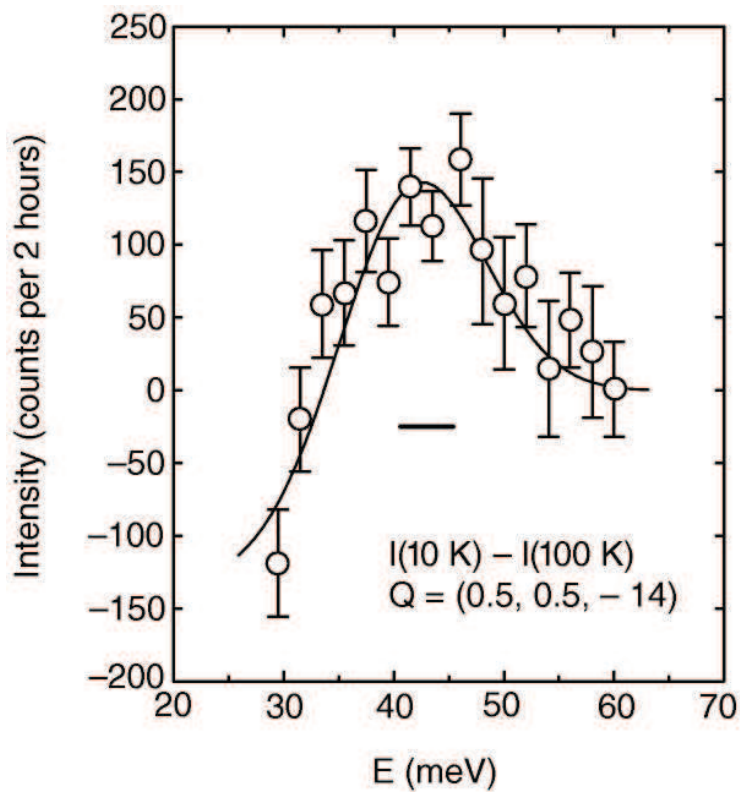

FIG. 12. Difference spectrum of the neutron scattering intensities from $\mathrm{Bi}_{2} \mathrm{Sr}_{2} \mathrm{CaCu}_{2} \mathrm{O}_{8+\delta}\left(\mathrm{T}_{c}=91\right.$ $\mathrm{K})$ at $T=10 \mathrm{~K}$ and $100 \mathrm{~K}$ at wavevector $Q=(\pi / a, \pi / a)$ showing the spin resonance at $\sim 43 \mathrm{meV}$. The horizontal bar represents the instrumental energy resolution and the solid curve is a guide to the eye (after Fong et al $\stackrel{77}{ }$ ). 

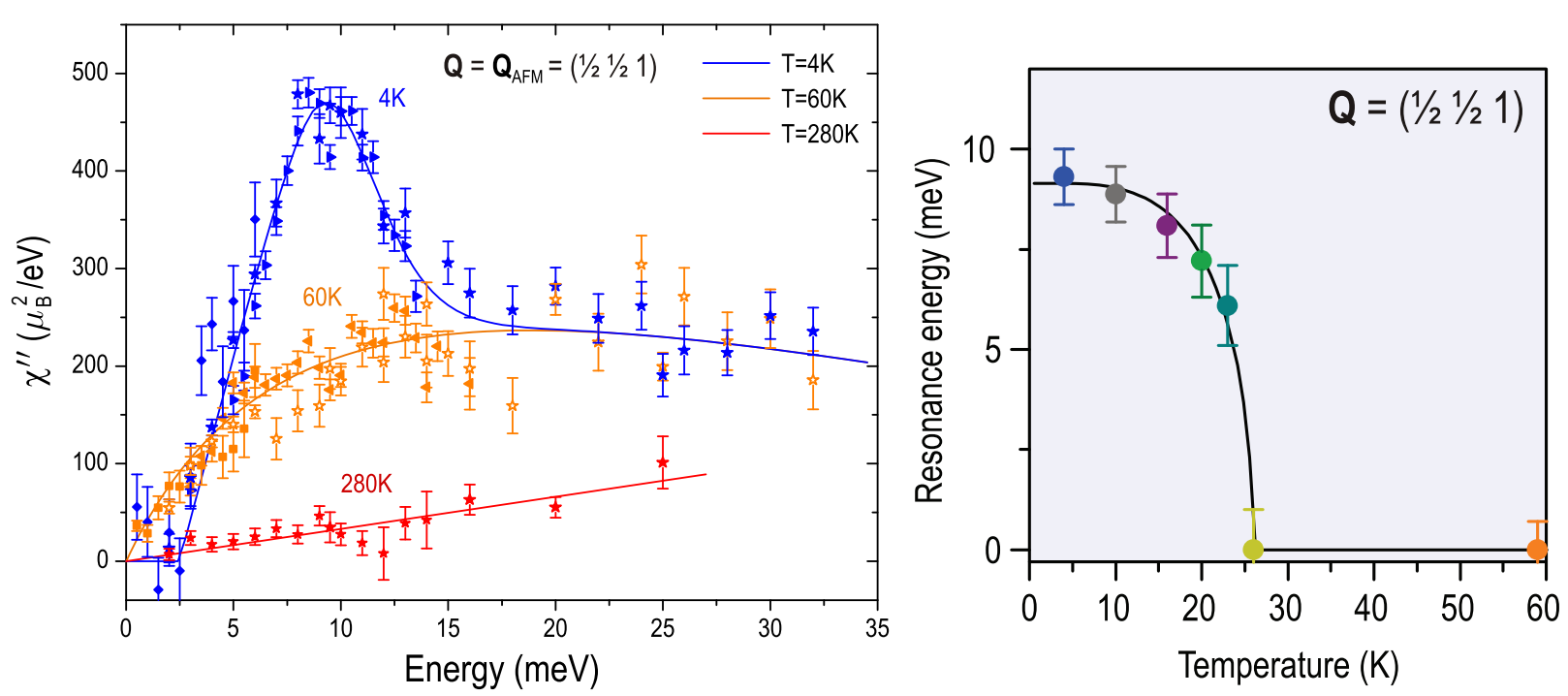

FIG. 13. (left) The neutron scattering spin resonance for $\mathrm{BaFe}_{1.85} \mathrm{Co}_{0.15} \mathrm{As}_{2}\left(\mathrm{~T}_{c}=26 \mathrm{~K}\right)$; (right) The energy of the resonance versus temperature follows a BCS-like curve (after Inosov et al. ${ }^{81}$ ).

momentum $Q$ which contribute significantly to the spin scattering 85,86

$$
\operatorname{Sgn}(\Delta(k+Q))=-\operatorname{Sgn}(\Delta(k))
$$

In this case the coherence factor Eq. (3) goes to 1 near threshold while if there were a plus sign in Eq. (4), it would vanish.

Equation (4) defines the class of unconventional superconductors which are the subject of this review. Materials in this class have a gap that changes sign on different parts of the Fermi surface or surfaces separated by a momentum $Q$ which connects regions which play an important role in the scattering of the electrons. Thus "unconventional" as used in this review is not related to the symmetry of the gap, nor is it determined by whether the gap has nodes or is nodeless. For example, the gap may have $A_{1 g}(s$-wave) symmetry but change sign between two different pieces of the Fermi surface, as the so-called $s^{ \pm}$-gap proposed for the Fe-pnictides ${ }^{87}$. As discussed in Sec. IV, such an $A_{1 g}$ gap can also have nodes ${ }^{88}$. Alternatively, one could have a $B_{1 g}$ ( $d$-wave) nodeless gap on multiple Fermi surfaces.

\section{MODELS}

In this section we introduce the basic models that will be discussed. While these are certainly minimal models, we will argue that they exhibit a number of the important physical 
properties which are observed in the actual materials. On this basis, it is reasonable to examine the structure of the pairing interaction in these models as will be done in Sec. IV]

As illustrated in Fig. 5, these materials have crystal structures consisting of layers containing square planar arrays of $d$ - or $f$-electron cations embedded in an anion lattice. Here we will take a minimal approach which focuses on the $d$ or $f$ electrons and treats the anion lattice as providing a crystalline electric field and a hybridization network. This misses the charge-transfer character ${ }^{89}$ of the $\mathrm{CuO}_{2}$ planes, the dynamic polarization effects of anions such as As, and the spd conduction bands of the heavy fermion and actinide anions. However, as we will discuss, we believe that this approach captures the essential physics that leads to pairing in these materials.

In outline, this approach begins with the selection of local $d$ or $f$ atomic states for the $(\mathrm{Cu}, \mathrm{Fe}, \mathrm{Ce}, \mathrm{Pu})$ ions which takes account of the appropriate crystal-field and spin-orbit couplings. Then these states are hybridized through the (O, As, In, Ga) anion states, or directly, leading to a tight binding band or bands. The tight binding hopping parameters are typically adjusted so that the low energy states fit the results of bandstructure calculations. For the heavy fermion and actinide systems, one includes a further phenomenological renormalization. Here one has the Kondo physics to deal with and the approximation is based on the assumption that just as in the single-ion case, the system renormalizes to a heavy Fermi liquid. Then an onsite Coulomb interaction and, if there are multiple orbitals, additional inter-orbital Coulomb and exchange interactions are added. Even at this level, there are various parameterizations which involve the choice of basis states for the bandstructure calculation, and the Wannier projection of the bands in the vicinity of the Fermi energy onto the local orbital basis ${ }^{90}-92$.

Then of course, when a model is selected, one needs to determine its properties. There have been a number of different theoretical approaches used to determine the properties of Hubbard models. Analytic or semi-analytic methods have included random phase approximations (RPA) ${ }^{93}-\underline{96}$, renormalized meanfield theory (RMFT) ${ }^{97} \underline{-99}$, conserving fluctuation exchange (FLEX) ${ }^{100}-102$, self-consistent renormalization (SCR) $)^{103}$, two-particle-self-consistent (TPSC) ${ }^{104}$, and slave-boson approximations ${ }^{105}-107$. Numerical approaches include deter-

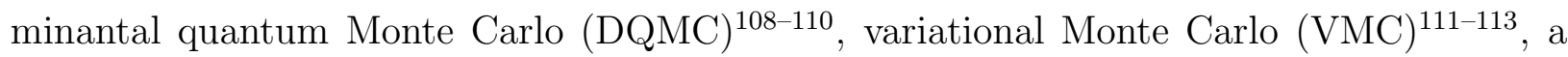
variety of cluster Monte Carlo (CDMFT $\stackrel{114}{ }$, $\mathrm{DCA}^{115}$, $\left.\mathrm{VCPT}^{116}\right)$ methods, density matrix renormalization group (DMRG) $\frac{117}{17}$ calculations as well as functional renormalization group 
(FRG) $)^{\underline{54}-\underline{57}}$ studies. Our goal in this section is to introduce the Hubbard models that have been used to describe the unconventional superconductors and illustrate some of the results for their physical properties which have been found from numerical calculations.

\section{A. The cuprates}

To illustrate the type of models that we have in mind, and discuss some of their properties, we begin with the cuprates. At the $\mathrm{Cu}$ site, the crystal field splitting pushes the $\mathrm{Cu}$ $d_{x^{2}-y^{2}}$ orbit up in energy so that it contains the last $(3 \mathrm{~d})^{9}$ electron of $\mathrm{Cu}^{2+}$. The undoped system with one hole per $\mathrm{Cu}$, is a charge-transfer antiferromagnetic insulator with a gap set by the energy to move the hole from a $\mathrm{Cu}$ to a neighboring $\mathrm{O}$. The large onsite $\mathrm{Cu}$ Coulomb interaction leads to well formed $S=1 / 2$ moments on the $\mathrm{Cu}$ which are coupled by a $\mathrm{Cu}-\mathrm{O}-\mathrm{Cu}$ superexchange interaction ${ }^{36}$. A weak interlayer exchange coupling leads to a Néel transition with a checkerboard antiferromagnetic spin arrangement in the $\mathrm{CuO}_{2}$ plane. When a material such as $\mathrm{La}_{2-x} \mathrm{Sr}_{x} \mathrm{CuO}_{4}$ is hole doped by adding $\mathrm{Sr}$, the antiferromagnetism is rapidly suppressed and below a temperature $T^{*}$ one enters a pseudogap phase. This phase is believed to reflect the approach to the Mott state and provides a medium in which a variety of instabilities can appear as the temperature is lowered. These continue to be studied and among other correlations are believed to contain fluctuating charge and $\pi$-phase shifted antiferromagnetic stripes ${ }^{42}$ which at low temperatures may order leading to a reconstruction of the Fermi surface $e^{118}-120$ or if disordered form a spin glass $\frac{121}{}$. While evidence of superlattice order does appear in some underdoped cuprates $\left(\mathrm{La}_{1.875} \mathrm{Ba}_{0.125} \mathrm{CuO}_{4}{ }^{58}\right)$, there are others, including ordered stoichiometric crystals $\left(\mathrm{YBa}_{2} \mathrm{Cu}_{4} \mathrm{O}_{8}{ }^{122}\right)$ in which a pseudogap appears in the apparent absence of a translational broken symmetry. This has led to various interesting theoretical proposals of Fermi surface reconstruction without translational symmetry breaking 123,124 . In the overdoped regime the system is metallic with a large Fermi surface and spin-fluctuations.

Early on, Anderson suggested that a minimal model which contained the essential cuprate physics was the single band Hubbard model. In this case, one focuses on the $\mathrm{Cu} \mathrm{d}_{x^{2}-y^{2}}$ orbital and hybridizes it through the $\mathrm{O}$ anion network leading to a single $d_{x^{2}-y^{2}}$ band. Then adding 
an onsite Coulomb interaction $U$, one has the well known 2D single band Hubbard model 225 .

$$
H=-\sum_{i j s} t_{i j}\left(d_{i s}^{+} d_{j s}+d_{j s}^{+} d_{i s}\right)+U \sum_{i} n_{i \uparrow} n_{i \downarrow}
$$

Here $t_{i j}$ are tight binding one-electron hopping parameters between sites $i$ and $j$ which are adjusted to fit the bandstructure and $U$ is an onsite Coulomb interaction. In Eq. (5), $d_{i s}^{+}$ creates an electron with spin $s$ in a $d_{x^{2}-y^{2}}$ orbital on the $i$ th site, $d_{j s}$ destroys one on the $j$ th site and $n_{i \uparrow}=d_{i \uparrow}^{+} d_{i \uparrow}$ is the occupation number for a spin up electron on the $i$ th site.

Although the single-band Hubbard model, Eq. (5), is certainly a minimal model, it exhibits a number of the basic phenomena which are seen in the cuprate materials. At halffilling, in the strong coupling limit it maps to the 2D spin $1 / 2$ Heisenberg model on a square lattice. Numerical studies of the Heisenberg model ${ }^{126}$ find evidence of long range antiferromagnetic order at $T=0$. In addition, analytic calculations 127,128 have provided the basis for understanding a range of experimental results for the undoped cuprates. Alternatively in weak coupling, it has been shown ${ }^{129}$ that the doped Hubbard model has a transition to a $d_{x^{2}-y^{2}}$ superconducting phase. While this result was obtained in the limit $U / t \rightarrow 0$, it establishes the fact that this simple model can exhibit a $d_{x^{2}-y^{2}}$ superconducting phase.

As noted, there have been a variety of numerical approaches used to study the Hubbard model. At half-filling, the particle-hole symmetry eliminates the so-called "fermion sign" problem for a Hubbard model with a near-neighbor one-electron hopping. In this case, determinant quantum Monte Carlo (DQMC) ${ }^{108}$ calculations can be carried out on large lattices down to low temperatures. These calculations find that the half-filled 2D Hubbard model with a near neighbor hopping $t$ and an onsite Coulomb interaction $U$ of order the bandwidth $8 t$ is a Mott insulator and has a groundstate with long range antiferromagnetic $\operatorname{order}^{109}$. In addition, in this intermediate coupling regime where $U$ is of order the bandwidth, one sees both the local and itinerant character of the magnetism. Figure 14 shows Monte Carlo results for the square of the $z$-component of the local moment $m_{z}(\ell)=n_{\ell \uparrow}-n_{\ell \downarrow}$ versus temperature for a range of $U / t$ values ${ }^{110}$. As expected, when the temperature decreases below a scale set by $U,\left\langle m_{z}^{2}\right\rangle$ increases. However, at a lower temperature scale $\left\langle m_{z}^{2}\right\rangle$ is found to increase further for weak coupling, while it decreases for strong coupling. In the weak coupling itinerant case, this increase is associated with the formation of short range particlehole magnetic correlations. In this case, the energy gain at low temperatures is proportional to $\left\langle m_{z}^{2}\right\rangle$ so that $\left\langle m_{z}^{2}\right\rangle$ increases further as $T$ decreases. Alternately, in the strong coupling 


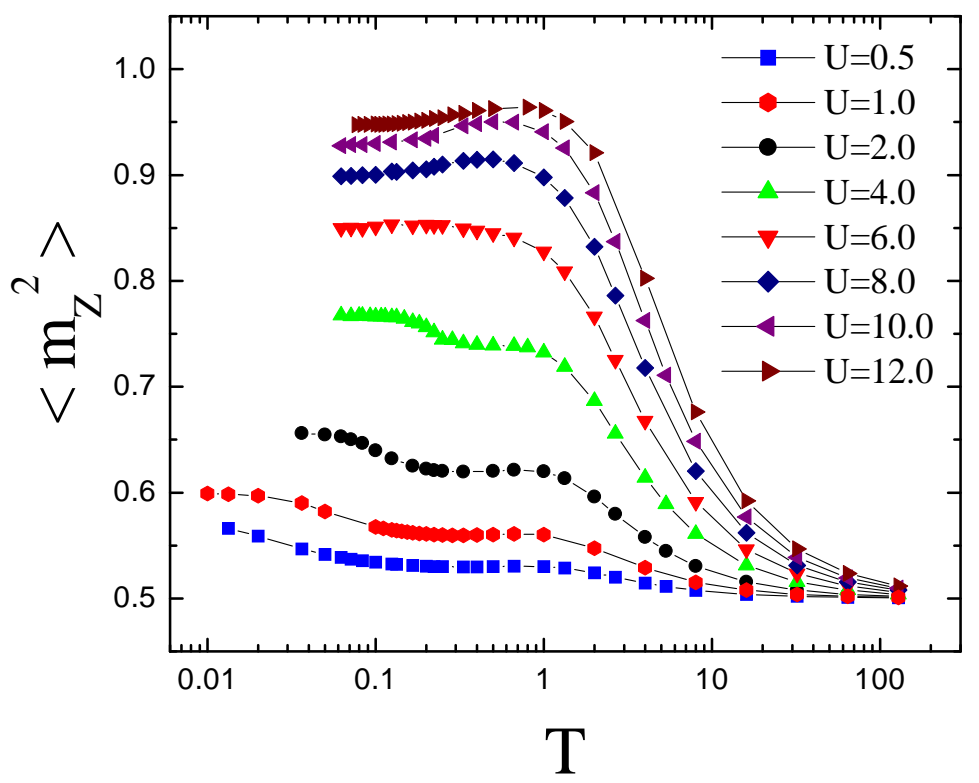

FIG. 14. The temperature dependence of the square of the local moment for different values of the on-site Coulomb repulsion $U$ (in units where $t=1$ ). As the temperature decreases below $\sim U / 2$, local onsite correlations lead to an increase in $\left\langle m_{z}^{2}\right\rangle$. Then on a lower temperature scale, non-local spin correlations develop and for weak coupling $\left\langle m_{z}^{2}\right\rangle$ increases, while for strong coupling it decreases. This crossover marks a change from an itinerant to a more local magnetic behavior (after Paiva et al. $\frac{110}{}$ ).

case, below an energy scale $U$ one has well-defined local moments. In this case, as the temperature decreases further and drops below the exchange energy $J \sim 4 t^{2} / U$, virtual electron transfer associated with $J$ reduces the degree of localization and $\left\langle m_{z}^{2}\right\rangle$ decreases. As seen in Fig. 14 the crossover between this local moment and itinerant behavior occurs for a value of $U$ of order the bandwidth. As we will see, it is in this intermediate coupling parameter regime, where the system has both local and itinerant characteristics, that the doped system has its highest $T_{c}$.

For the doped Hubbard model the fermion sign problem limits the temperatures that are accessible using the DQMC approach and alternative numerical approximations have been developed. Using a Gutzwiller projected $d$-wave BCS wavefunction ${ }^{97}$, variational Monte Carlo (VMC) calculations have been used to explore the $T=0$ phase diagram of the doped $x=1-\langle n\rangle$ Hubbard mode ${ }^{112}$. The groundstate is found to be a $d$-wave superconductor for 
$0<x<x_{c}$ with $x_{c} \approx 0.35$. For $x>x_{c}$, the groundstate is a Landau-Fermi liquid. At low doping $(x \lesssim 0.1)$ Gutzwiller projected wavefunctions with both $d$-wave and antiferromagnetic variational parameters have been found to have a lower energy than the $d$-wave alone, providing evidence for a coexisting antiferromagnetic and $d$-wave superconducting phase $\frac{113}{}$. These VMC calculations find results for the doping dependence of the coherence length, the penetration depth as well as the momentum distribution in agreement with experimental observations.

An alternative approach to dealing with the doped case is represented by various cluster methods. Here, the basic idea is to treat the degrees of freedom within a cluster exactly and take into account the correlations beyond the cluster by introducing a self-consistent dynamic mean-field. The resulting problem of a cluster embedded in a dynamic meanfield is then solved by means of exact disgonalization for small clusters or by various Monte Carlo approaches such as the Hirsch-Fye algorithm 130 for larger clusters. The coupling of the cluster to the self-consistent dynamic meanfield significantly reduces the fermion sign problem. In the so-called cellular dynamic mean-field theory (CDMFT) 114 and the variational cluster-perturbation theory $(\mathrm{VCPT})^{116}$ methods, the system is mapped onto an embedded cluster in real space while in the dynamic cluster approximation (DCA) 115 the cluster is embedded in reciprocal space. This latter scheme keeps the periodic boundary conditions and coarse grains the Brillouin zone, making it a convenient approach for studying the momentum dependence of the pairing interaction.

There are also functional renormalization group (FRG) approaches ${ }^{131}$, so named because they follow the flow of the four-point vertex function $\Gamma\left(k_{1}, k_{2}, k_{3}, k_{4}\right)$ for scattering between states on the Fermi surface as the states outside an energy $\Delta E$ of the Fermi energy are integrated out. Here, the degrees of freedom are reduced to states in a $\Delta E$-shell around the Fermi surface. This shell is then discretized into a finite number of Fermi surface patches which allows one to take into account the tangential momentum dependence of the effective interaction. In practice, the renormalization group equations are typically carried out at the one-loop level. The resulting coupled renormalization group equations are then numerically integrated to determine the functional renormalization group flow of the scattering vertex as the energy cut-off $\Delta E$ or temperature is reduced. Although the one-loop approximation means that it is necessary to start the system off with appropriate bare interactions and stop the calculations when the renormalized interaction grows too large, this approach can 
provide an unbiased treatment of competing instabilities and can indicate which instability or combination of instabilities are important. There have also been proposals in which the FRG is used down to a given cut-off where the most divergent parts of $\Gamma\left(k_{1}, k_{2}, k_{3}, k_{4}\right)$ are then taken to construct a low energy reduced Hamiltonian ${ }^{132}$, which can then be solved using exact diagonalization.

The density matrix renormalization group (DMRG) ${ }^{117}$ has also been used to study these models. This approach has primarily been implemented as a real space renormalization procedure in which degrees of freedom are iteratively added, for example by increasing the size of the lattice system. Then the less important degrees of freedom are truncated from the Hilbert space by keeping only a finite number of the most probable eigenstates of a reduced density matrix. This iterative, variational method is designed to thin the degrees of freedom to those which play the dominant role in the ground state. It has proved particularly effective for one-dimensional ladder models.

Using these approaches, further evidence has been found that the Hubbard models exhibit many of the basic physical properties which characterize the unconventional superconductors. Specifically, for the doped systems there is evidence for antiferromagnetic spinfluctuations, pseudogap behavior, nematic correlations, $d$-wave or more generally unconven-

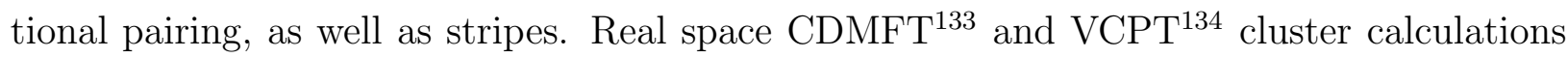
find clear signatures of antiferromagnetic, pseudogap and $d$-wave behavior in the Hubbard model. Including longer range one-electron hopping, these calculations find ground state phase diagrams and single particle spectral weights for electron- and hole-doping that are similar to the overall behavior observed in these materials. A small orthorhombic distortion of the one-electron hopping is found to lead to a large nematic response ${ }^{135}$. Similarly, momentum space DCA calculations find evidence for pseudogap behavior in the spin susceptibility and the single particle spectral weight $\frac{136}{}$ as well as nematic correlations $\frac{137}{}$. Using the DCA and a sequence of different clusters $\stackrel{138}{ }$, Maier et al. $\stackrel{139}{ }$ found evidence shown in Fig. 15 for the divergence of the $d$-wave pairfield susceptibility

$$
P_{d}(T)=\int_{0}^{1 / T}\left\langle\Delta_{d}(\tau) \Delta_{d}^{+}(0)\right\rangle d \tau
$$

for a doped Hubbard model. Here $\Delta_{d}^{+}=\frac{1}{2 \sqrt{N}} \sum_{\ell, \delta}(-1)^{\ell} d_{\ell \uparrow}^{+} d_{\ell+\delta \downarrow}^{+}$with $\delta$ summed over the four near neighbor sites of $\ell$.

FRG studies of the single band Hubbard model with a next near neighbor hopping $t^{\prime}$ find 
dopings for which the interaction vertex flows to antiferromagnetic or $d$-wave dominated regimes as well as a region of intermediate doping in which the forward scattering Pomeranchuck Fermi surface instabilities and CDW as well as nematic fluctuations grew $\underline{\underline{54}} \underline{-56}$. In this latter region, umklapp processes are found to play an important role linking the instabilities in various channels. In the underdoped regime, Lauchli et al..$^{132}$ have used the FRG to construct a low-energy effective Hamiltonian and argue that umklapp processes truncate Fermi surface segments leading to a psedogap phase. FRG calculations have also been carried out for the multi-orbital Hubbard models 56,57 . Here the geometry of the electron- and hole-Fermi surfaces (see Fig. [16 of the next section) lead to $\operatorname{SDW}(\pi, 0)$ and $s^{ \pm}$pairfield dominated flow regimes along with other umklapp mediated scattering processes 140,141 .

Calculations using the density matrix renormalization group (DMRG) to study the 2-leg Hubbard ladder find a spin gapped state at half-filling and power law $d$-wave-like pairfield correlations for the doped system ${ }^{142}$. As discussed in Sec. IVB, a twisted version of this same 2-leg ladder mimics the SDW stripe structure and $s^{ \pm}$pairing correlations seen in the Fe-based superconductors ${ }^{143}$. Calculations for a doped 6-leg Hubbard ladder ${ }^{144,145}$ find striped charge-density- $\pi$-phase-shifted-antiferromagnetic states like that shown in Fig. 15b. While short range $d_{x^{2}-y^{2}}$ pairfield correlations along the stripes were also observed there were no long range $d$-wave pairing correlations. In this case, periodic boundary conditions were used for the 6-site direction and open end boundary conditions along the direction of the legs forming a cylindrical tube. On an 8-leg $t-J$ system $\frac{146}{}$ the favored filling was 0.875 and the $\pi$-phase shifted antiferromagnetic striped structure was similar to that shown Fig. 15b with each cylindrical stripe containing four holes corresponding to a half-filled stripe. This is the same pattern which is observed in $\mathrm{La}_{1.875} \mathrm{Ba}_{0.125} \mathrm{CuO}_{4}{ }^{147}$. In these calculations, the tube-like boundary conditions favor the formation of cylindrical stripes. The short length of the circumference of the tube suppresses pair fluctuations between the stripes and leaves only short range $d$-wave pairing correlations along a stripe. With open boundary conditions and applied fields to orient the stripes along the long direction of the 6- and 8-leg ladders that have been studied, pair fluctuations between the stripes become possible and a stronger $d$-wave pairing response is observed. While present DMRG calculations find that the antiphase $d$-wave state is slightly higher in energy than that of the in-phase state, VMC calculations found parameter ranges in which the antiphase state was stabilized $\underline{61}$. There are also calculations for a coupled ladder model that exhibit stripes with antiphase pairing 60 . 

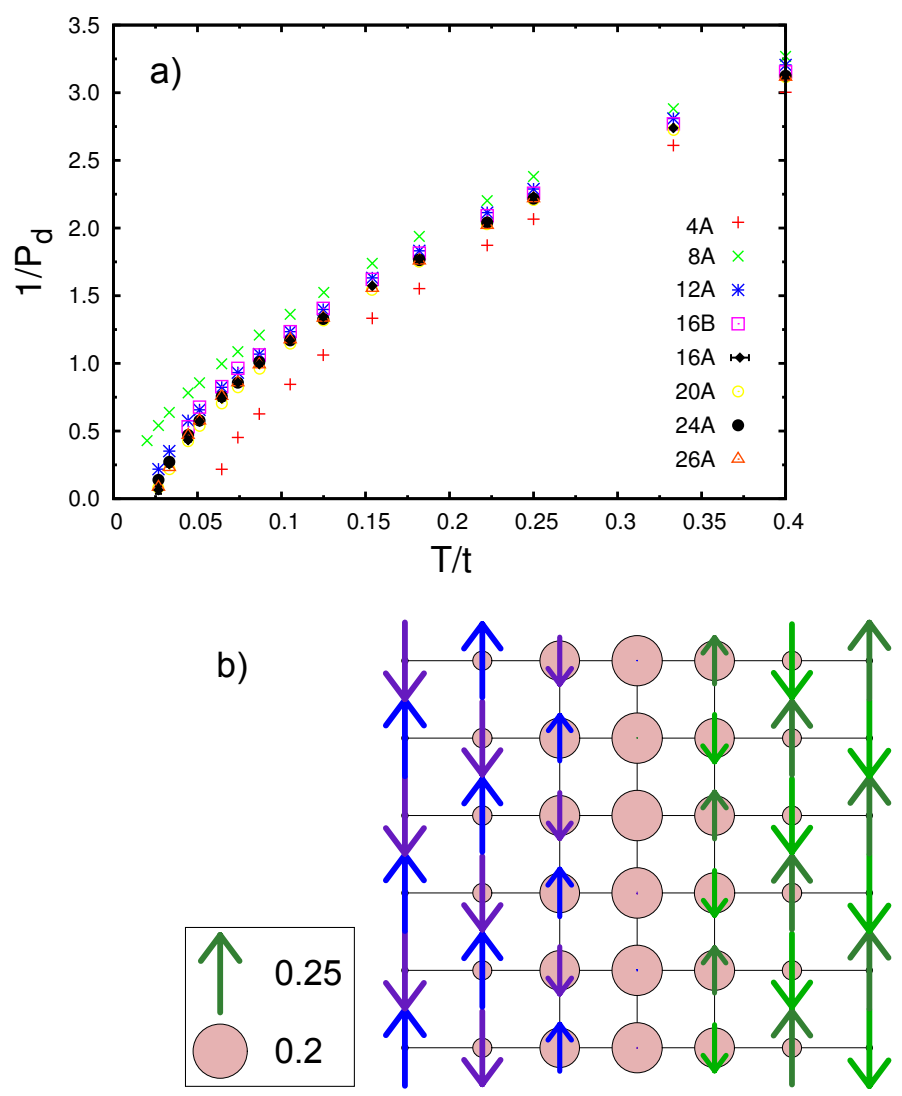

FIG. 15. $d_{x^{2}-y^{2}}$ pairing and stripes have been found in various numerical calculations for the doped Hubbard model. (a) DCA results for the inverse of the $d_{x^{2}-y^{2}}$-wave pairfield susceptibility versus $T / t$ for various sized Betts ${ }^{138}$ clusters. Here, $U / t=4$ and $\langle n\rangle=0.9$ (after Maier et al. ${ }^{139}$ ).

The charge $\left\langle n_{\text {hole }}(\ell)\right\rangle$ and spin $\left\langle S^{z}(\ell)\right\rangle$ structure seen in a DMRG calculations of a cylindrical 6-leg Hubbard model with $U / t=12$ (after White et al. $\stackrel{144}{ }$ and Hager et al. $\stackrel{145}{ }$ ).

Finally, along with the observations of $d$-wave and stripe correlations, there is numerical evidence of pseudogap behavior in the underdoped Hubbard model. A variety of dynamic cluster Monte Carlo calculations of the single particle spectral weight $136,148,149$ show the emergence of pseudogap behavior in the underdoped $t-t^{\prime}-U$ Hubbard model. A phenomenological theory of the pseudogap phase by Yang et al. $\stackrel{123}{ }$ has had success in reproducing many of the observed properties of the pseudogap regime.

The important point for the present discussion is that while the choice of the variational wavefunction in the VMC and finite size effects for the cluster calculations can influence what one finds, there is overall agreement among these various approaches that Hubbard models exhibit many of the basic physical properties which characterize the unconventional 
superconductors 150,151 . There are of course phenomena such as the unusual ordered magnetic phase in the underdoped cuprates observed in polarized neutron scattering experiments 152 and dichroic angular resolved photoemission measurements 153 which have not yet been found in these basic Hubbard models. Here we take the view that these phenomena are peripheral to the pairing mechanism.

\section{B. The Fe-pnictides}

The undoped Fe-pnictide materials have partially filled 3d shells and are antiferromagnetic metals below $T_{N}$. Their magnetic moments alternate in alignment row to row creating a stripe-like antiferromagnetic pattern different from the checkerboard pattern of the cuprates. Just above, or in some cases coinciding with, $T_{N}$ there is a tetragonal to orthorhombic lattice transition. As the system is doped, both the structural and the Néel transitions are suppressed and superconductivity occurs 154 .

For the Fe-pnictide superconductors, photoemission 155 as well as band structure calculations $156-158$ find that the states associated with the pnictide $4 \mathrm{p}$ orbitals are located some $2 \mathrm{eV}$ or more below the Fermi level. Thus an effective tight binding model based on the five Fe 3d orbitals can provide a reasonable description of the electronic states near the Fermi surface. Since the crystal field splitting, as well as the exchange and spin-orbit splittings of the iron $3 \mathrm{~d}$ orbitals are small relative to the bandwidth, all five $3 \mathrm{~d}$ orbitals need to be taken into account. For the 1111 materials the 3D coupling between the Fe layers is relatively weak and 2D models have proved useful. Due to the tetrahedral coordination of the pnictide, the unit cell contains two Fe sites. However, the Fe-pnictide plane is invariant under a reflection and a translation since each Fe has the same local arrangement of the surrounding atoms. Thus for the 2D Fe-pnictide layer one can unfold the Brillouin zone and work with an effective five-orbital model on a square lattice with one Fe per unit cell $\stackrel{159}{ }$. Including one-electron hopping parameters to describe both the direct Fe-Fe hopping as well as the hybridized hopping through the pnictide or chalcogen $4 p$ orbits, one arrives at a 5-band model with the one electron part of the Hamiltonian given by ${ }^{96}, 160$

$$
H_{0}=\sum_{i j} \sum_{\ell n \sigma} t_{i j}^{\ell n} c_{i \ell \sigma}^{+} c_{j n \sigma}+\sum_{i} \sum_{\ell \sigma} \varepsilon_{\ell} n_{i \ell \sigma}
$$

Here $\ell=(1,2, \ldots 5)$ denotes the Fe- $d$ orbitals $\left(d_{x z}, d_{y z}, d_{x y}, d_{x^{2}-y^{2}}, d_{3 z^{2}-r^{2}}\right)$ and $c_{i \ell \sigma}^{+}$creates 
an electron on site $i$ in the $\ell$ th orbit with spin $\sigma$. The tight binding parameters $t_{i j}^{\ell n}$ describe the one-electron hopping from the $\ell$ th orbit on site $i$ to the $n$th orbit on site $j$ and $\varepsilon_{\ell}$ is the site energy of the $\ell$ th orbit. The onsite Coulomb and exchange interaction part of the Hamiltonian is

$$
\begin{aligned}
H_{1}=\sum_{i} & \left(\sum_{\ell} U n_{i \ell \uparrow} n_{i \ell \downarrow}+U^{\prime} \sum_{\ell^{\prime}<\ell} n_{i \ell} n_{i \ell^{\prime}}\right. \\
& \left.-J \sum_{\ell \neq \ell^{\prime}} \mathbf{S}_{i \ell} \cdot \mathbf{S}_{i \ell^{\prime}}+J^{\prime} \sum_{\ell \neq \ell^{\prime}} c_{i \ell \uparrow}^{+} c_{i \ell \downarrow}^{+} c_{i \ell^{\prime} \downarrow} c_{i \ell^{\prime} \uparrow}\right)
\end{aligned}
$$

with $n_{i \ell}=n_{i \ell \uparrow}+n_{i \ell \downarrow}$ and $\mathbf{S}_{i \ell}=\frac{1}{2} c_{i \ell \sigma}^{+} \boldsymbol{\sigma}_{\sigma \sigma^{\prime}} c_{i \ell \sigma^{\prime}}$. Here $U$ and $U^{\prime}$ are the intra- and inter-orbital Coulomb interactions, $J$ is the Hund's rule exchange and $J^{\prime}$ the so-called pair hopping term. If these interactions are generated from a two-body term with spin rotational invariance $U^{\prime}=U-2 J$ and $J^{\prime}=J$. However, many body interactions can renormalize these couplings altering these relations. In addition the dressed interaction terms can in general depend on the orbital indices.

The $\mathrm{Fe}^{+2}$ ion separation $\sim 2.7^{\circ} \mathrm{A}$ is significantly smaller than the $\mathrm{Cu}^{+2}$ separation of $\sim 3.8^{\circ} \mathrm{A}$ and the direct Fe-Fe hopping along with the d-p hybridization through the pnictogen or chalcogen anions leads to a metallic groundstate. Observation of quantum oscillations provide clear evidence of well defined small Fermi surfaces consistent with a semi-metallic bandstructure ${ }^{33}$. The basic structure of the Fermi surfaces of the Fe-based superconductors consists of two electron cylinders at the zone corner of the $2 \mathrm{Fe}$ per unit cell Brillouin zone compensated by two or three hole sections around the zone center. The Fermi surface sheets for a two-dimensional five-orbital tight-binding fit $\frac{96}{\underline{6}}$ of the DFT bandstructure ${ }^{158}$ of LaOFeAs are shown in Fig. 16a. Here and in the following an unfolded 1Fe per unit cell Brillouin zone will be used. Diagonalizing the 5-orbital tight-binding Hamiltonian of Eq. (8), one has for the Bloch states of the $\nu$ th band,

$$
\Psi_{\nu \sigma}(k)=\sum_{\ell}\langle\nu k \mid \ell\rangle c_{\ell \sigma}(k)
$$

where, again, $\ell$ sums over the Fe orbitals $\left(d_{x z}, d_{y z}, \cdots\right)$ and $c_{\ell \sigma}(k)=\sum_{\mathbf{i}} c_{\mathbf{i} \ell \sigma} e^{i(\mathbf{k} \cdot \mathbf{i})} / \sqrt{N}$. The main orbital weight contributions $|\langle\nu k \mid \ell\rangle|^{2}$ to the band states that lie on the various Fermi surfaces are indicated by the colors in Fig. 16a. A more detailed look at the orbital weights is shown in Fig. 16b, where they are plotted as a function of the winding angle on the different Fermi surfaces. Here one sees, for example, that the $d_{y z}$ and $d_{x y}$ orbitals contribute 

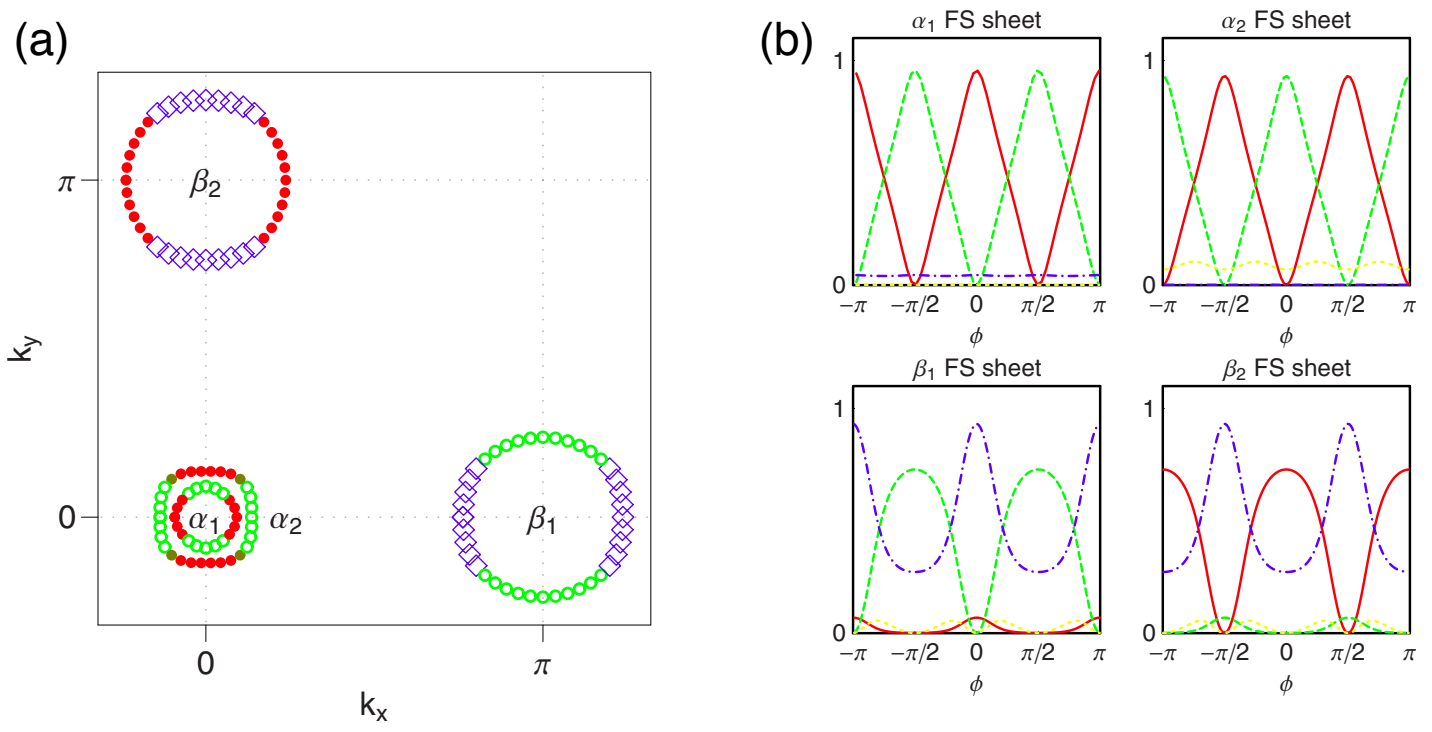

FIG. 16. This figure illustrates the Fermi surfaces and orbital weight distributions for a 5-orbital model of the 1111 Fe-based superconductors. (a) The Fermi surface sheets of a 5-orbital tight binding model of the 1111 Fe-based superconductors. The symbols (color online) denote the orbital which has the largest orbital weight, with the $d_{x z}$ (red solid circles $\bullet$ ), $d_{y z}$ (green open circles $\circ$ ), and $d_{x y}$ (blue open diamonds $\diamond$ ). (b) The orbital weights as a function of winding angle $\phi$ on the various Fermi surface sheets with $d_{x z}$ (solid red), $d_{y z}$ (dashed green), $d_{x y}$ (dash-dot blue) and $d_{x^{2}-y^{2}}$ (short dashed yellow). The $d_{3 z^{2}-r^{2}}$ orbital weight is negligible. Here, the $d_{x z}$ and $d_{y z}$ orbitals are aligned along the Fe-Fe directions (after Graser et al.$^{96}$ ).

the dominant weights on the $\beta_{1}$ electron pocket while it is the $d_{x z}$ and $d_{y z}$ that mainly contribute to the $\alpha$ pockets. These orbital weights play an important role in determining the strength and structure of the pairing interaction.

While the 1111 materials can be reasonably treated as two-dimensional, the structure of the 122 systems is such that one needs to take their three dimensionality into account. The loss of the reflection-translation invariance of the 2D layer leads to more complex 10-orbital models $\frac{161}{\text {. }}$ 


\section{The heavy fermion materials}

The heavy fermion materials have incomplete $f$-shells and there is a balance between the strong onsite Coulomb interactions which tend to localize the $f$-electrons and the hybridization with extended bandstates of the ligand anions which delocalize them. At high temperatures the system exhibits local moment behavior with magnetic moments of order atomic values while at low temperatures the system resembles a Fermi liquid with large quasi-particle masses associated with the hybridized $f$-electrons. In the coexisting state where one has both SDW antiferromagnetism and superconductivity, the magnitude of the ordered moments determined from neutron scattering and the effective mass of the paired electrons, determined from the specific heat jump at $T_{c}$, are large. Thus the $f$ electrons play an important role in both the antiferromagnetism and the superconductivity $\underline{162}, \underline{163}$.

Hotta and Ueda introduced a minimal model for such an $f$-electron system based on a $j-j$ coupling scheme since the spin-orbit interaction is large. In addition they noted that this provided a convenient way to define the one-electron states that make up the pairs. The resulting Hamiltonian for the 115 Ce heavy fermion superconductors has a form similar to Eqs. (7) and (8) but with the one electron operators describing Kramer's doublets and with $\sigma$ a pseudospin quantum number. The 14 -fold degenerate $f$ electronic states are split by the spin-orbit coupling into a low lying $j=5 / 2$ sextet and a higher energy $j=7 / 2$ octet. For $\mathrm{Ce}^{3+}$ with a $(4 f)^{1}$ configuration, only the $j=5 / 2$ sextet contributes to the electronic states near the Fermi energy. The one electron states of the $j=5 / 2$ sextet are further split by the crystalline electric field of the In ligand anions, separating the six $j=5 / 2$ states into three sets of Kramer's doublets. For a tetragonal crystal field one has

$$
\begin{array}{cc}
c_{i n \sigma}^{+}=p f_{i \pm 5 / 2}^{+}+q f_{i \mp 3 / 2}^{+} & n=1 \\
-q f_{i \pm 5 / 2}^{+}+p f_{i \mp 3 / 4}^{+} & n=2 \\
f_{i \pm 1 / 2}^{+} & n=3
\end{array}
$$

Here $f_{i m}^{+}$creates an electron on the $i$ th lattice site in a $j=5 / 2$ orbital with a $z$-component of total angular momentum $m$. The "orbital" index $n=1,2$ and 3 denotes the $\left\{\Gamma_{7}, \Gamma_{7}^{\prime}, \Gamma_{6}\right\}$ tetragonal field Kramer's doublets, the $q$ and $p$ coefficients in Eq. (10) depend on the tetragonal crystalline field and $\sigma= \pm 1$ is the pseudospin quantum number.

As schematically illustrated in Fig. 5 , the spacing $\sim 4.6^{\circ} \mathrm{A}$ of the $\mathrm{Ce}^{3-}$ ions is the largest of the three systems and the $4 f$ electrons of $\mathrm{Ce}^{3-}$ tend to be localized. Thus as opposed to 
the itinerant 3d-electrons of the Fe-based materials and the doped cuprates, the $f$-electrons of the heavy fermion 115 materials are nearly localized. The materials are metallic because of the $4 p$ states of the anions and the dispersion of the $4 f$ electrons arises from their hybridization with these $4 p$ conduction electrons. As in both the Fe-pnictide and the doped cuprates, quasi-two-dimensional Fermi surfaces have been observed in de Haas-van Alphen experiments $\frac{164}{6}$ for the Ce compounds. Similarly to the Fe-based superconductors, the heavy fermion materials have multiple Fermi surfaces and there are orbital weight factors associated with the $\Gamma_{7}, \Gamma_{7}^{\prime}$ and $\Gamma_{6}$ orbital states.

As previously discussed, the plutonium intermetallic compounds PuMGa $a_{5}$ have the same tetragonal structure as the cerium-based heavy fermion 115 superconductors. Electronic structure calculations $\frac{165}{}$ for $\mathrm{PuCoGa}_{5}$ show a similarity between the main Fermi surfaces of $\mathrm{CeCoIn}_{5}$ and $\mathrm{PuCoGa}_{5}$. In particular, there are $f$-electron dominated cylindrical Fermi surface hole sheets centered at the $\Gamma$ point, and cylindrical electron sheets centered at the $M$ point of the $1 \mathrm{Fe}$ per unit cell Brillouin zone. Using the $j-j$ coupling scheme to construct a low energy model for this actinide superconductor, Maehira et al. $\underline{.165}$ noted that the $\mathrm{Pu}-115$ compound is the hole version of Ce-115. That is, the low lying $j=5 / 2$ sextet accommodates the one $(4 f)$ electron of $\mathrm{Ce}^{3+}$ for $\mathrm{CeCoIn}_{5}$, while it has one hole for the $(5 f)^{5} \mathrm{Pu}^{3+}$ ion in $\mathrm{PuCoGa}$. This picture of the $\mathrm{Pu}-115$ compound being a hole version of the Ce-115 compound is particularly striking for $\mathrm{PuCoIn}_{5}$ and $\mathrm{CeCoIn}_{5}$.

Finally, while the existence and, to a reasonable degree, the structure of Fermi surfaces of the heavy fermion $\frac{166}{}$ and the Fe-based 167,168 superconductors are well established, the situation for the cuprates is still debated $\frac{169}{}$. In the overdoped single layer cuprate $\mathrm{Tl}_{2} \mathrm{Ba}_{2} \mathrm{CuO}_{6+\delta}$ (Tl2201) both angle-dependent magnetoresistance $\frac{170}{}$ and ARPES measurements $\frac{171}{2}$ provide evidence for a large quasi-two-dimensional Fermi surface in reasonable agreement with bandstructure calculations. More recently $\underline{172}$, the observation of quantum oscillations in the magnetoresistance and the magnetization of Tl2201 provided direct evidence of this large hole-like Fermi surface and coherent fermionic excitations. Here, the observation of quantum oscillations are important in determining that coherent excitations are present. Following the development of highly ordered $\mathrm{YBa}_{2} \mathrm{Cu}_{3} \mathrm{O}_{6.5}$ (ortho-II) crystals $\frac{173}{\text {, quantum oscillations }}$ were also observed in the underdoped regime, both in the Hall resistance $\frac{174}{4}$ and in the magnetization 175,176 . This showed that the doped cuprates, just as the heavy fermion and Fe superconducting materials, can have a Fermi surface with low-lying fermionic excitations, 
even in the underdoped regime. The fact that the Hall and Seebeck coefficients are negative indicates that the observed small Fermi-surface pockets are electron-like 177,178 . The large Fermi surface of the overdoped cuprates must therefore undergo a reconstruction as the doping level decreases $\frac{179}{2}$. One mechanism for such a reconstruction is the occurrence of some new periodicity associated with an ordered phase such as a spin striped phase 180,181 or a unidirectional charge density wave $\frac{119}{}$. NMR measurements show that high magnetic fields induce charge order without spin order $\frac{182}{2}$ which would be consistent with a unidirectional charge density wave. Some studies $\underline{182} \underline{\underline{184}}$ attribute the Fermi-surface reconstruction in $\mathrm{YBa}_{2} \mathrm{Cu}_{3} \mathrm{O}_{y}$ to a form of stripe order similar to that observed in $\mathrm{La}_{2} \mathrm{CuO}_{4}$-based cuprates $\frac{185}{185}$ and there is evidence for a phase transition at $T^{*}$ associated with some form of density wave $\frac{178}{}$ or nematic $\frac{186}{18}$ order leading to a pseudogap phase $\frac{187}{18}$. A recent compilation $\frac{188}{2}$ of ARPES measurements, high magnetic field quantum oscillation studies and transport experiments suggests that a small $Q$ wave-vector bidirectional charge density wave provides an explanation for the nodal Fermi surfaces which is consistent with a wide variety of complementary measurements.

\section{THE PAIRING INTERACTION}

In this section, we examine the structure of the pairing interaction for the models discussed in Sec. III. The pairing interaction is given by the irreducible particle-particle fourpoint vertex. As discussed in the appendix, for the conventional superconductors this interaction is well described by a phonon exchange and screened Coulomb interaction. In general, for spin rotationally invariant models, the irreducible particle-particle vertex can be separated into a fully irreducible vertex and $S=1$ spin and $S=0$ charge (particle-hole) exchange channels. For the 2D Hubbard model near half-filling, DCA calculations find that the $S=1$ spin channel gives the dominant contribution to the pairing. Similarly, for the two-layer Hubbard model introduced in this section, it is the $S=1$ spin fluctuation channel that leads to pairing. However, as discussed, it can lead to $B_{1 g}$ ( $d$-wave) or $A_{1 g}$ ( $s$-wave) pairing depending upon the structure of the Fermi surface. This bilayer Hubbard model, as well as a "twisted ladder" model discussed in this section, illustrate the link between the

cuprate and Fe-based superconductors. For the multi-band Fe-based superconductors one has only weak coupling results, but here the resulting phenomenology provides evidence that 
the pairing is driven by the spin fluctuations and similarly for the heavy fermion models where it is the pseudo-spin fluctuations. The conclusion is that the pairing in the models of Sec. III is mediated by spin-fluctuations.

As discussed in Appendix A, the momentum and frequency dependence of the superconducting gap provide information on the space-time structure of the pairing interaction $\frac{189}{}$. For conventional superconductors such as $\mathrm{Pb}$ or $\mathrm{Hg}$, the gap is weakly dependent upon momentum but strongly frequency dependent, implying that the pairing interaction is short range and has a retarded part. As is well known, electron tunneling 190 and optical absorption $\frac{191}{}$ measurements of the frequency dependence of the gap for the low $T_{c}$ materials identify the pairing interaction as arising from a retarded phonon-mediated contribution and an "instantaneous" repulsive screened Coulomb term. For the unconventional superconductors, a determination of both the momentum and frequency dependence of the gap are important. Here a wide variety of experiments have been used to probe the momentum dependence of the gap. These include ARPES $\underline{192} \stackrel{-196}{=}$, phase sensitive tunneling experiments $\frac{197}{2} \underline{-199}$, Raman scattering 200,201 , low temperature thermal conductivity $\underline{202}$ and directional magnetic field specific heat measurements ${ }^{38}$. There have also been various tunneling $203-206$ and optical studies of the frequency dependence of the gap $\underline{207} \underline{209}$. Thus, at present, there are a range of experimental results and interpretations. From many of these it appears that for the unconventional superconductors one is dealing with a pairing interaction that peaks at a large momentum transfer characteristic of the near-neighbor antiferromagnetic or SDW correlations and which has a frequency response characteristic of the spectrum of the antiferromagnetic spin fluctuations. However, there are questions and controversies regarding this $\underline{210} \underline{213}$ and it remains a challenge to obtain the close interplay between experiment and theory that was the hallmark for the traditional superconductors. Furthermore, a complete range of measurements for the heavy fermion and Fe-based materials, comparable to the results for the cuprates, are not yet available.

With this in mind, this review has the more limited goal of understanding the momentum, frequency and orbital structure of the interaction that is responsible for pairing in the models discussed in Sec. III. To the extent that these models exhibit the basic low energy properties which are found in these materials, one can argue that the interaction responsible for pairing in the models will reflect the pairing interaction in the real materials.

In this section, we will show dynamic cluster approximation (DCA) results for the pairing 
interaction. The basic assumption of the DCA is that the self-energy and irreducible vertex functions are short-ranged and can be well represented by a finite size cluster. Under this assumption, one sets up an effective cluster problem as an approximation for the bulk thermodynamic limit in order to calculate these quantities. This is done by representing the bulk lattice by an effective cluster embedded in a mean-field bath, which is designed to represent the remaining degrees of freedom and is determined self-consistently. In contrast to other finite size methods, in which one carries out calculations on finite size lattices and then tries to scale up in size, the DCA, for a given cluster size, gives approximate results for the bulk thermodynamic limit.

The DCA treats spatial correlations on length scales within the cluster accurately and non-perturbatively and describes longer-ranged correlations on a mean-field level. It becomes exact in both the weak-coupling $(U / t=0)$ and strong-coupling $(t / U=0)$ limits. For finite $U / t$, one can in principle obtain exact results by carrying out calculations for different size clusters and then extrapolating to infinite cluster size. Convergence with cluster size depends on the specific problem, but is usually faster than with finite size methods, because of the inclusion of the remaining degrees of freedom in terms of a mean-field. This was discussed for the 3D half-filled Hubbard model in Kent et al.214 and Fuchs et al. $\underline{215}$, where the accuracy of the DCA was benchmarked against finite size methods for several different quantities. In particular, it was shown that well converged results for the antiferromagnetic $T_{N}$ versus $U$ phase diagram can be obtained from relatively small clusters. As noted, in this approach the cluster is embedded in reciprocal space and one obtains momentum space results on a coarse grained Brillouin zone. It is convenient to work in momentum space and since the pairing interaction is expected to be short-ranged it is actually more amenable to cluster calculations than the long-range pairfield correlations. Like the FRG calculations, the DCA provides an unbiased treatment of the competing instabilities. In addition, it takes account of self-energy and interaction effects within the cluster while treating the remaining degrees of freedom within a dynamic meanfield.

\section{A. The single band Hubbard model}

For the single band Hubbard model DCA numerical simulations have been used to determine the momentum and frequency dependence of the pairing interaction $\stackrel{216}{ }$. Formally, this 
interaction is given by the irreducible particle-particle scattering vertex $\Gamma^{p p}\left(k, k^{\prime}\right)$ shown on the left-hand side of Fig. 17. It consists of all Feynman diagrams that can not be separated

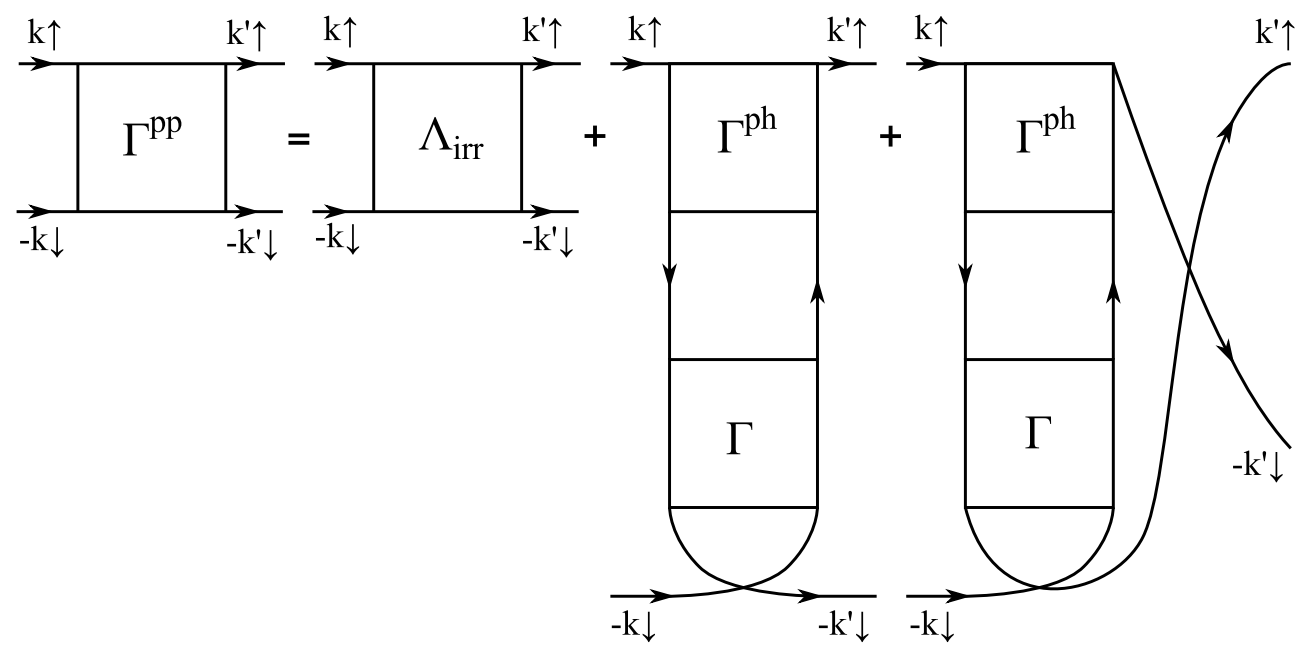

FIG. 17. The pairing interaction is given by the irreducible particle-particle vertex $\Gamma^{p p}$. Here $\Gamma^{p p}$ is decomposed into a fully irreducible two-fermion vertex $\Lambda_{\text {irr }}$ plus contributions from the $S=1$ and $S=0$ particle-hole channels. $\Gamma^{p h}$ are irreducible particle-hole vertices, $\Gamma$ is the full vertex and the solid lines are fully dressed single particle propagators.

into two parts by cutting just two particle lines. Here, $k=\left(\mathbf{k}, i \omega_{n}\right)$ with $\omega_{n}=(2 n+1) \pi T$ a fermion Matsubara frequency and one is interested in the scattering of a pair in a singlet, zero center-of-mass momentum and energy state with relative momentum and Matsubara frequency $k=\left(\mathbf{k}, i \omega_{n}\right)$ to a final state with $k^{\prime}=\left(\mathbf{k}^{\prime}, i \omega_{n^{\prime}}\right)$. Results obtained from a 64-site $8 \times 8$ numerical dynamic cluster approximation $(\mathrm{DCA})$ for $\Gamma^{p p}\left(k, k^{\prime}\right)$ with $\omega_{n}=\omega_{n^{\prime}}=\pi T$ at a filling $\langle n\rangle=0.85$ and $U=4 t$ are shown on the right hand side of Fig. 188 ${ }^{217}$ Here one sees that as the temperature is lowered, the singlet pairing interaction increases for large momentum transfers. This is a reflection of the growth of the short range antiferromagnetic spin-fluctuations as seen in a similar plot of the spin susceptibility $\chi(q)$ shown on the left hand side of Fig. 18, Taking the Fourier transform of $\Gamma^{p p}\left(k, k^{\prime}\right)$

$$
\Gamma^{p p}\left(\ell_{x}, \ell_{y}\right)=\frac{1}{N} \sum_{k k^{\prime}} e^{i k \cdot \ell} \Gamma^{p p}\left(k, k^{\prime}\right) e^{i k^{\prime} \cdot \ell}
$$

leads to the real space picture of the pairing interaction illustrated in Fig. 19, Here $\Gamma^{p p}\left(\ell_{x}, \ell_{y}\right)$ is the strength of the $\omega_{n}=\omega_{n^{\prime}}=\pi T$ pairing interaction between a singlet formed with one electron at the origin and the other at site $\left(\ell_{x}, \ell_{y}\right)$. It is large and repulsive if the electrons 

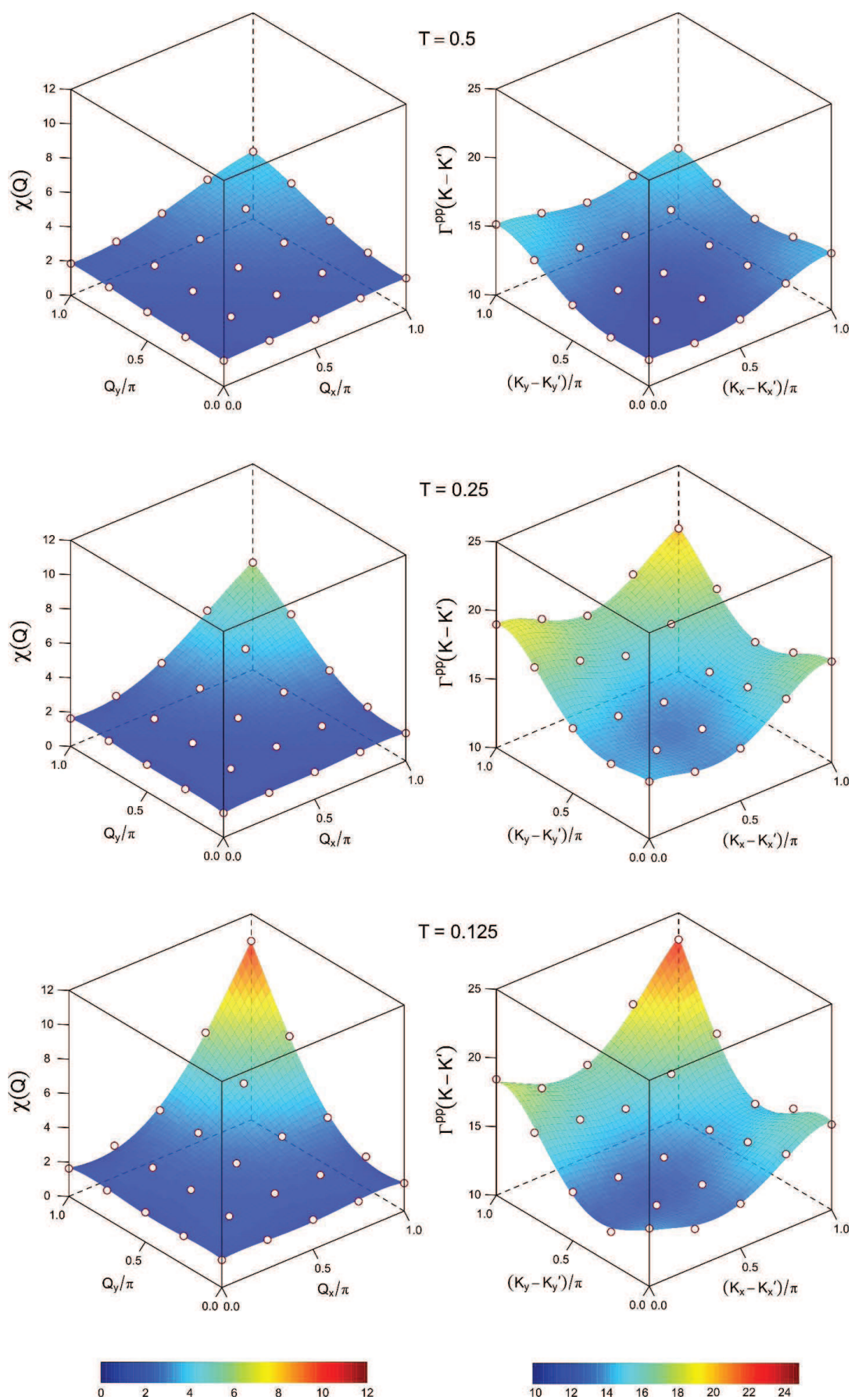

FIG. 18. The spin susceptibility $\chi(q)$ and the pairing interaction $\Gamma^{p p}\left(K, K^{\prime}\right)$ for $U=4 t$ and $\langle n\rangle=$ 0.85 are compared at various temperatures. As the temperature is reduced a peak develops in $\Gamma^{p p}$ reflecting the peak in $\chi$. This repulsive peak is the origin of the unconventional superconductivity discussed in this review. 


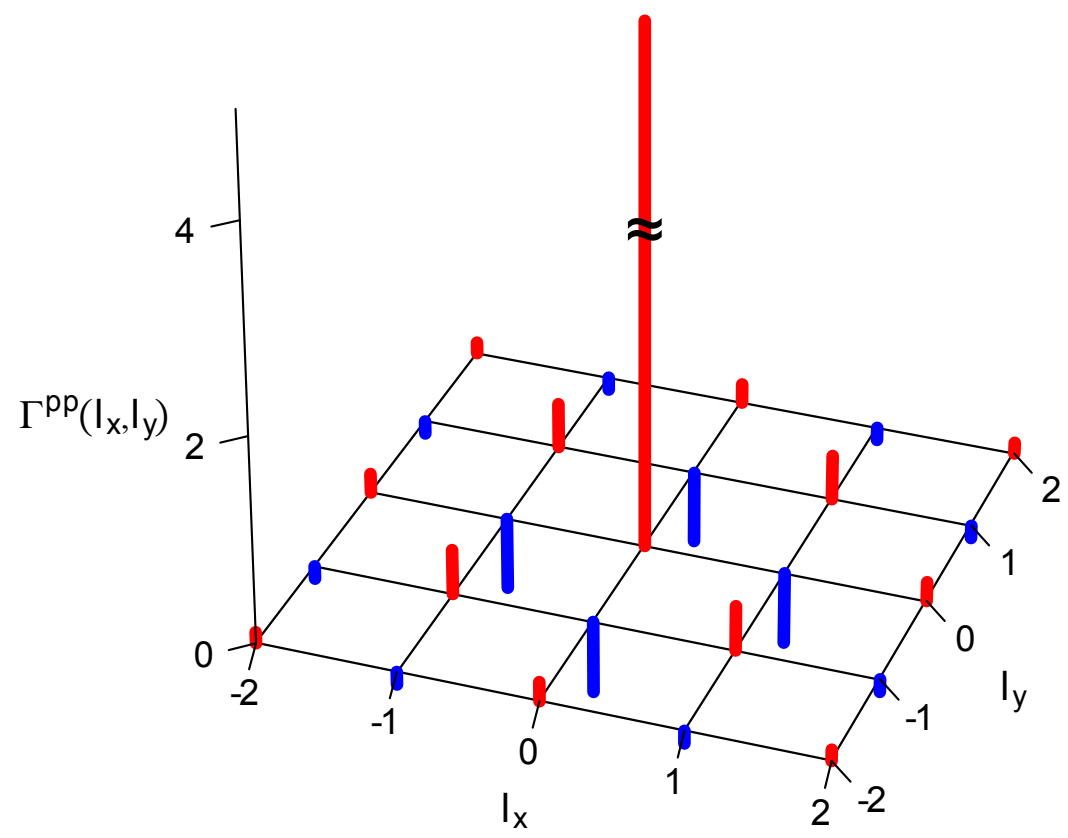

FIG. 19. The real space structure of the pairing interaction obtained from the Fourier transform Eq. (11) of $\Gamma^{p p}\left(k, k^{\prime}\right)$ at a temperature $T=0.125 t$ for $U=4 t$ and $\langle n\rangle=0.85$. Here red indicates a repulsive and blue an attractive pairing interaction for a singlet formed between an electron at the origin and an electron at site $\left(\ell_{x}, \ell_{y}\right)$. The peak in $\Gamma^{p p}$ shown in Fig. 18 leads to a pairing interaction which oscillates in space.

occupy the same site but attractive if they are on near neighbor sites reflecting the peaking of $\Gamma^{p p}\left(k, k^{\prime}\right)$ for $k-k^{\prime} \sim(\pi, \pi)$.

As shown in Fig. 17, the pairing interaction $\Gamma^{p p}\left(k, k^{\prime}\right)$ can be separated into a fully irreducible two-fermion vertex $\Lambda_{\text {irr }}$ and partially reducible particle-hole exchange contributions. Here the fully irreducible part $\Lambda_{\text {irr }}$ is defined as the sum of all diagrams that can not be separated into two pieces by cutting any combination of two lines (particle or hole). For a spin rotationally invariant system, the particle-hole exchange contributions appearing on the right hand side of Fig. 17 can be combined into an $S=1$ magnetic spin fluctuation piece $\frac{3}{2} \Phi_{m}$ and a spin $S=0$ charge density fluctuation contribution $\frac{1}{2} \Phi_{d}$.

$$
\Gamma^{p p}\left(k, k^{\prime}\right)=\Lambda_{\text {irr }}\left(k, k^{\prime}\right)+\frac{3}{2} \Phi_{m}\left(k, k^{\prime}\right)+\frac{1}{2} \Phi_{d}\left(k, k^{\prime}\right)
$$

Carrying out a DCA calculation, one can evaluate the individual terms that enter Eq. (12). 
The upper left panel of Fig. 20 shows the pairing interaction $\Gamma\left(k, k^{\prime}\right)$ versus momentum
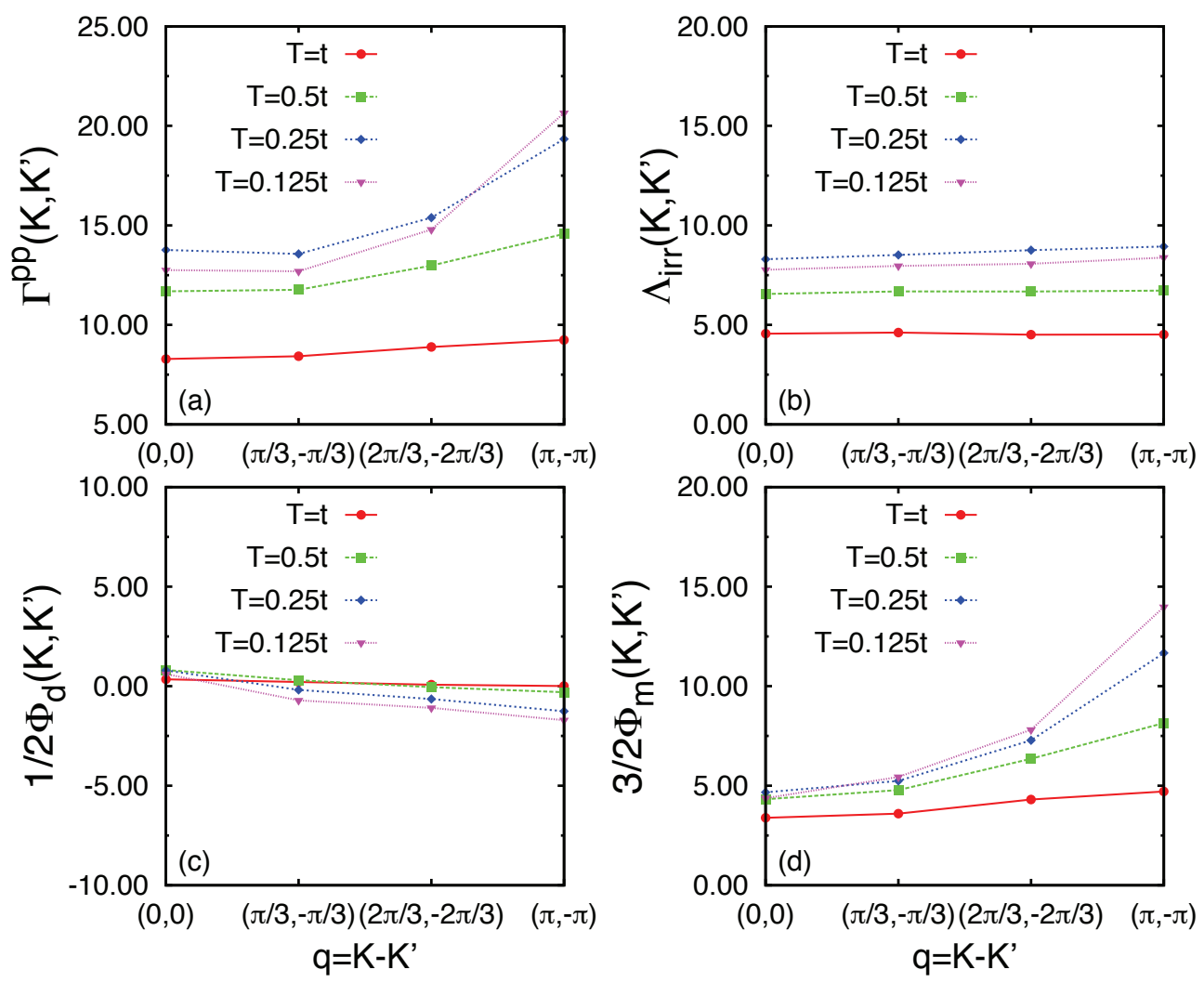

FIG. 20. This figure illustrates the momentum dependence of the various contributions that make up the irreducible particle-particle pairing vertex $\Gamma^{p p}$. (a) The irreducible particle-particle vertex $\Gamma^{p p}$ versus $q=K-K^{\prime}$ for various temperatures with $\omega_{n}=\omega_{n^{\prime}}=\pi T$. Here, $K=(\pi, 0)$ and $K^{\prime}$ moves along the momentum values of the 24-site cluster which lay on the dashed line shown in the inset of Fig. 21, Note that the interaction increases with the momentum transfer as expected for a $d$-wave pairing interaction. (b) The $q$-dependence of the fully irreducible two-fermion vertex $\Lambda_{\text {irr }}$. (c) The $q$-dependence of the charge density $(S=0)$ channel $\frac{1}{2} \Phi_{d}$ for the same set of temperatures. (d) The $q$-dependence of the magnetic $(S=1)$ channel $\frac{3}{2} \Phi_{m}$. Here, one sees that the increase in $\Gamma^{p p}$ with momentum transfer arises from the $S=1$ particle-hole channel (after Maier et al. $\frac{216}{}$ ).

transfers along the diagonal $\left(k_{x}-k_{x}^{\prime}, k_{y}-k_{y}^{\prime}\right)$ of Fig. 18 for $\langle n\rangle=0.85$ and $U / t=4$ as the temperature is reduced. The remaining panels of Fig. 20 show the contributions of the fully irreducible vertex $\Lambda_{\text {irr }}$, the $S=0$ charge-fluctuations $\frac{1}{2} \Phi_{d}$ and the $S=1$ spin-fluctuations $\frac{3}{2} \Phi_{m}$. As noted, it is the increase of $\Gamma$ with momentum transfer that gives rise to the attractive near-neighbor pairing and it is clear from Fig. 20, that this comes from the $S=1$ 
part of the interaction. The fully irreducible vertex is essentially independent of momentum transfer and so it only contributes to the on-site repulsion, while the $S=0$ charge part decreases at large momentum giving rise to a small repulsive near neighbor interaction.

In these numerical calculations, one also obtains the dressed single particle Green's function $G\left(k, i \omega_{n}\right)$. Given $G$ and $\Gamma^{p p}$, one can determine the Bethe-Salpeter eigenvalues and eigenfunction in the particle-particle channel by solving

$$
-\frac{T}{N} \sum_{k^{\prime}} \Gamma^{P P}\left(k, k^{\prime}\right) G_{\uparrow}\left(k^{\prime}\right) G_{\downarrow}\left(-k^{\prime}\right) \phi_{\alpha}\left(k^{\prime}\right)=\lambda_{\alpha} \phi_{\alpha}(k) .
$$

This is basically the fully dressed BCS gap equation and when the leading eigenvalue goes to 1 the system becomes superconducting. One can also construct similar Bethe-Salpeter equations for the charge and magnetic particle-hole channels. Figure 21 shows a plot of the leading eigenvalues associated with the particle-particle pairing channel and the particle-

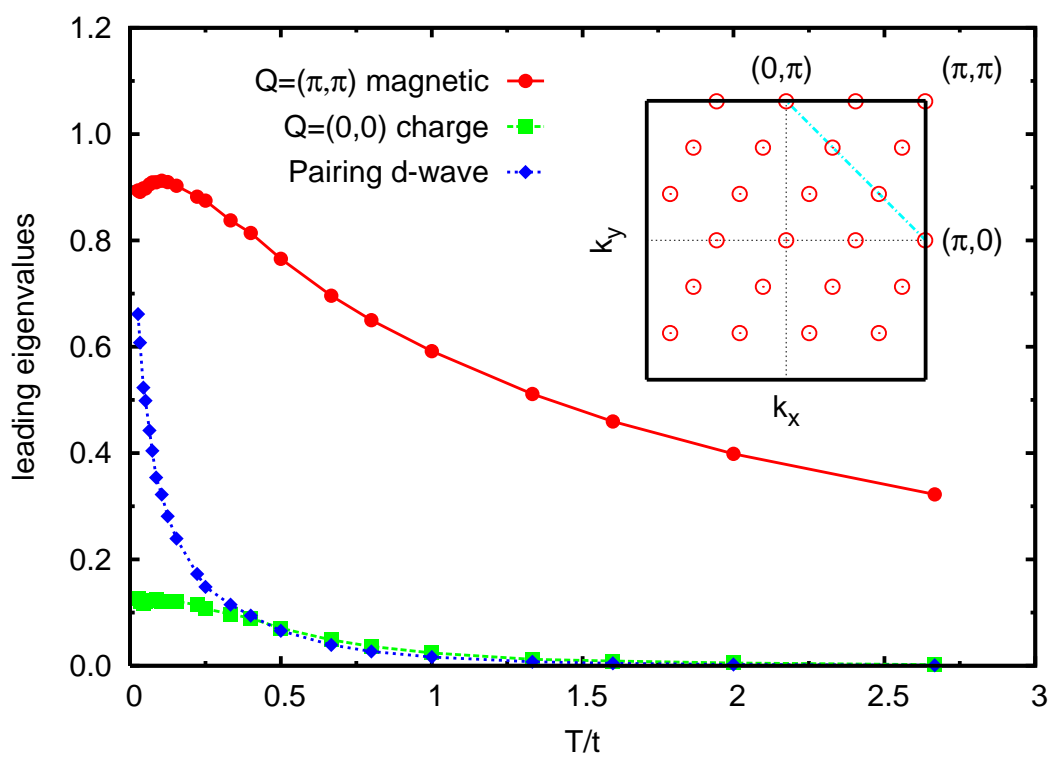

FIG. 21. Leading eigenvalues of the Bethe-Salpeter equation in various channels for $U / t=4$ and a site occupation $\langle n\rangle=0.85$. The $Q=(\pi, \pi), \omega_{m}=0, S=1$ magnetic eigenvalue is seen to saturate at low temperatures. The leading eigenvalue in the singlet $Q=(0,0), \omega_{m}=0$ particle-particle channel has $d_{x^{2}-y^{2}}$ symmetry and increases toward 1 at low temperatures. The largest charge density eigenvalue occurs in the $Q=(0,0), \omega_{m}=0$ channel and saturates at a small value. The inset shows the distribution of $k$-points for the 24 -site cluster (after Maier et al. ${ }^{216}$ ).

hole charge $S=0$ and spin $S=1$ channels for $U / t=4$ and a filling $\langle n\rangle=0.85$. As the temperature is lowered, the particle-hole $S=1$ antiferromagnetic channel with center 
of mass momentum $Q=(\pi, \pi)$ is initially dominant. However, at low temperatures the $Q=0$ pairing channel rises rapidly and the divergence of the antiferromagnetic channel saturates. The charge channel eigenvalue remains small. Thus one concludes that the pairing interaction arises from the exchange of $S=1$ particle-hole fluctuations.

The momentum dependence of the leading pairing eigenfunction $\varphi_{\alpha}(k)$ is shown in the

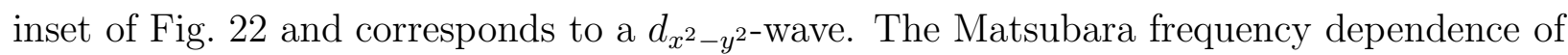

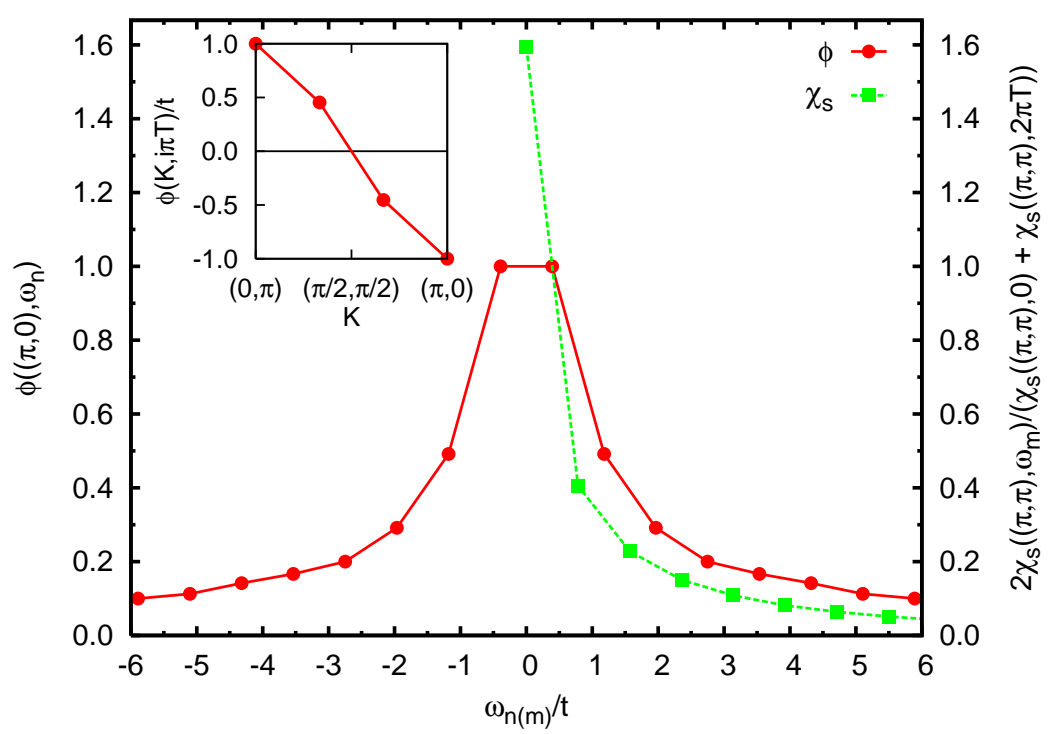

FIG. 22. The Matsubara frequency dependence of the eigenfunction $\phi_{d_{x^{2}-y^{2}}}\left(K, \omega_{n}\right)$ of the leading particle-particle eigenvalue of Fig. 21 for $K=(\pi, 0)$ normalized to $\phi(K, \pi T)$ (red, solid). Here, $\omega_{n}=(2 n+1) \pi T$ with $T=0.125 t$. The Matsubara frequency dependence of the normalized magnetic spin susceptibility $2 \chi\left(Q, \omega_{m}\right) /[\chi(Q, 0)+\chi(Q, 2 \pi T)]$ for $Q=(\pi, \pi)$ versus $\omega_{m}=2 m \pi T$ (green, dashed). The Matsubara frequency dependence of $\phi_{d_{x^{2}-y^{2}}}$ and the normalized spin $Q$ susceptibility are similar. Inset: The momentum dependence of the eigenfunction $\phi_{d_{x^{2}-y^{2}}}(K, \pi T)$ normalized to $\phi_{d_{x^{2}-y^{2}}}((0, \pi), \pi T)$ shows its $d_{x^{2}-y^{2}}$ symmetry. Here, $\omega_{n}=\pi T$ and the momentum values correspond to values of $K$ which lay along the dashed line shown in the inset of Fig. 21 (after Maier et al $\left.{ }^{216}\right)$.

this eigenfunction, shown in Fig. 22, has a similar decay to that of the spin susceptibility. However, as one knows, it is difficult to determine the real frequency response from limited numerical Matsubara data. Recent cellular dynamic meanfield studies by Kyung et al. $\stackrel{218}{\underline{2}}$ for real frequencies find a correspondence between the frequency dependence of the gap 
function and the local spin susceptibility as shown in Fig. 23. The frequency dependence of
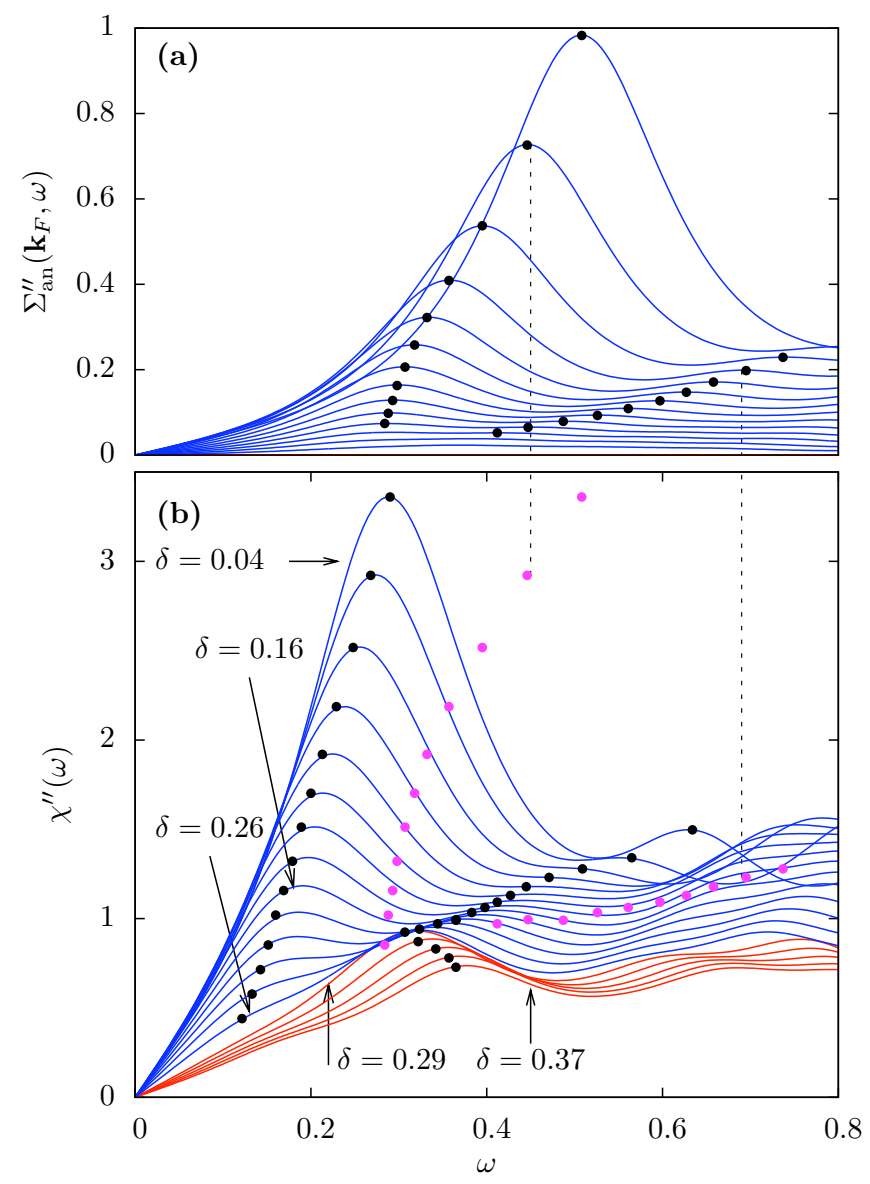

FIG. 23. This figure provides evidence linking the frequency dependence of the imaginary part of the gap function $\phi_{d}\left(\omega, k_{F}\right)$, which is called $\Sigma_{\text {an }}^{\prime \prime}\left(\omega, k_{F}\right)$ in this figure, to the frequency dependence of the spin fluctuation spectral weight $\chi^{\prime \prime}(\omega)$. (a) The imaginary part of the gap function $\Sigma_{\text {an }}^{\prime \prime}\left(\omega, k_{F}\right)$ at a wave vector $k_{F}$ near the antinode is plotted versus $\omega$ for various dopings $\langle n\rangle=1-\delta$. (b) The imaginary part $\chi^{\prime \prime}(\omega)$ of the local spin susceptibility versus $\omega$ for the same set of dopings. The black dots in (a) and (b) identify peaks. The position of the peaks of $\Sigma_{\text {an }}^{\prime \prime}$ in (a) are shown as the magenta dots in (b) at the same height as the corresponding $\chi^{\prime \prime}$ to illustrate their correspondence. One can see that the upward frequency shift of the $\Sigma_{\text {an }}^{\prime \prime}$ peaks relative to the $\chi^{\prime \prime}$ peaks decreases with the doping like the single particle gap. The red curves are for the normal state. Here, $U=8 t$, $t^{\prime}=-0.3 t^{\prime}, t^{\prime \prime}=-0.08 t$ and a Lorentzian broadening of $0.125 t$ was used for an embedded $2 \times 2$ plaquette (after Kyung et al: $\stackrel{218}{ }^{\text {}}$ ).

the interaction has also been discussed by Maier et al. ${ }^{219}$ and Hanke et al. ${ }^{220}$ who find that 
the dominant part of the interaction comes from the spectral region associated with spin fluctuations with an additional small contribution coming from high frequency excitations. All of these dynamic calculations are for small clusters so that it will be useful to have further work on the dynamics for larger clusters since it provides an important fingerprint of the pairing interaction.

At low temperatures where the leading eigenvalue $\lambda_{\alpha}$ of Eq. (13) approaches 1, the pairing interaction $\Gamma^{p p}\left(k, k^{\prime}\right)$ can be approximated as

$$
\Gamma^{p p}\left(k, k^{\prime}\right) \cong \varphi_{\alpha}(k) V_{\alpha} \varphi_{\alpha}\left(k^{\prime}\right)
$$

with a pairing strength $V_{\alpha}$

$$
V_{\alpha}=\frac{\sum_{k, k^{\prime}} \varphi_{\alpha}(k) \Gamma^{p p}\left(k, k^{\prime}\right) \varphi_{\alpha}\left(k^{\prime}\right)}{\left(\sum_{k} \varphi_{\alpha}^{2}(k)\right)^{2}} .
$$

Using Eq. (14), the inverse of the pairfield susceptibility is approximately given by

$$
P_{\alpha}^{-1} \cong P_{0 \alpha}^{-1}+V_{\alpha}
$$

with

$$
P_{0 \alpha}=\frac{T}{N} \sum_{k} G(k) G(-k) \varphi_{\alpha}^{2}(k) .
$$

Here $G(k)$ is the dressed single particle Green's function. For $d_{x^{2}-y^{2} \text {-wave pairing one has }}$ $\phi_{\alpha}(k) \sim\left(\cos k_{x}-\cos k_{y}\right)$ with a Matsubara frequency cut-off as seen in Fig. 222. As seen in Fig. 18, $\Gamma^{p p}\left(k, k^{\prime}\right)$ peaks for $\mathbf{k}-\mathbf{k}^{\prime} \sim(\pi, \pi)$ so that $V_{d}$ given by Eq. (15) is negative. One can think of $P_{0 \alpha}$ as the "intrinsic" $\alpha$-pairfield susceptibility of the interacting system.

In the traditional phonon mediated case, the pairing strength $V_{\alpha}$ is essentially independent of temperature once the ionic lattice is formed. Then the $N(0) \log \left(\omega_{D} / T\right)$ divergence of $P_{0 \alpha}$ gives a transition temperature $T_{c} \sim \omega_{D} e^{-1 / N(0)\left|V_{\alpha}\right|}$ where $P_{\alpha}^{-1}\left(T_{c}\right)=0$. For a strongly interacting system, both $P_{0 \alpha}$ and $V_{\alpha}$ are functions of temperature. As seen from the temperature dependence of $\Gamma^{p p}\left(k, k^{\prime}\right)$ in Fig. 18, the strength $\left|V_{d}(T)\right|$ of the interaction will increase as the temperature is lowered and $\chi(Q, T)$ increases. For the doped system, away from the antiferromagnetic instability, $\left|V_{d}(T)\right|$ will saturate to a constant value at low temperatures. However, as the doping $x$ goes to zero, it will continue to increase as the temperature decreases. In this case for $\langle n\rangle=1, P_{0 d}(T)$, Fig. 24, will be suppressed at low temperatures due to the vanishing of the quasi-particle weight as well as phase fluctuations $\underline{42}$ and $T_{c}$ will 


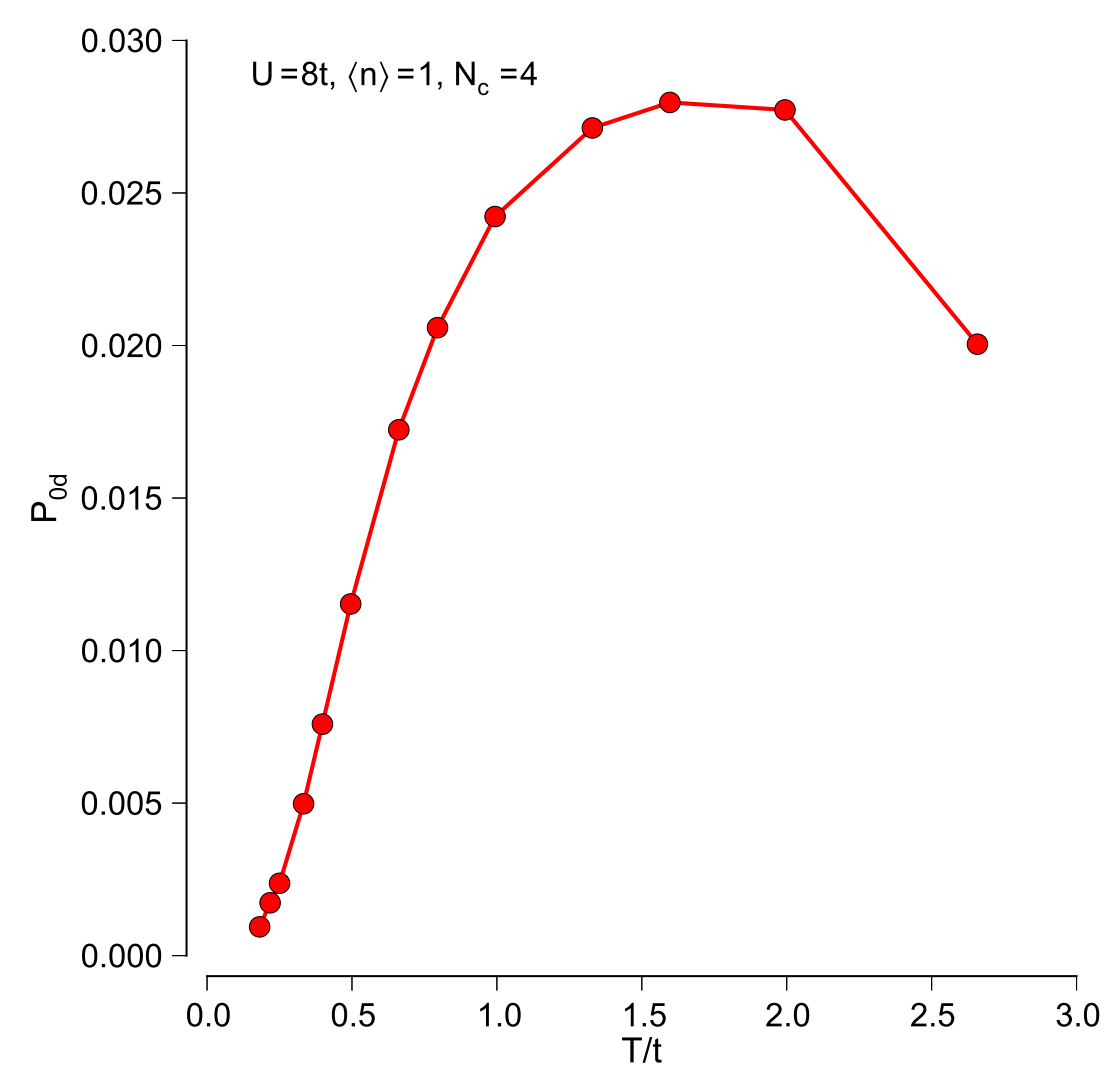

FIG. 24. The intrinsic pairfield susceptibility $P_{0 d}(T)$ for $U=8 t$ and $\langle n\rangle=1$ is suppressed as $T$ goes to zero (after Maier et al. $\stackrel{221}{ }$ ).

go to zero 221 . The interplay of the pairing strength $V_{\alpha}$, as $\langle n\rangle$ goes to 1 , and the intrinsic pairfield susceptibility $P_{0 d}$, which is suppressed as $\langle n\rangle$ goes to 1 , leads to a dome-shaped $T_{c}$ versus doping behavior. Thus while the strength of the pairing interaction can increase, the increased scattering leads to a reduction of the quasiparticle weight. In addition, it is important to remember that the pairing interaction is short range, of order the near-neighbor spacing. This is reflected in the $\left(\cos k_{x}-\cos k_{y}\right)$ structure of the gap. Thus it is not the correlation length of the antiferromagnetic correlations but rather having the spectral weight of the interaction in the right momentum and energy regime that determines the pairing strength.

The interplay of $P_{0 d}$ and the pairing interaction strength is of particular interest near a quantum critical point $\underline{73}, 124, \underline{222}$. Abanov et al. $\underline{223}$ have argued that the pseudogap phase reflects aspects of the pairing in the quantum-critical regime near the antiferromagnetic QCP. 
Recently, Metlitski and Sachdev $\stackrel{222}{2}$ have discussed the special role played by the competition between the spin density wave, Fermi surface structure and superconducting order in the two-dimensional system. In this case, while the quasiparticle spectral weight is suppressed at "hot spots" on the Fermi surface where $\varepsilon_{k+Q}=\varepsilon_{k}$, they find that the pairing interaction slightly away from the hot spots is strong and combined with a finite quasiparticle spectral weight can lead to high $T_{c}$ superconductivity.

Based on the similarity of the momentum and frequency dependence of $\Gamma^{p p}$ to that of the spin susceptibility $\chi$, approximate pairing interactions have been used in which

$$
\Gamma^{p p}\left(k, k^{\prime}\right) \simeq \frac{3}{2} \bar{U}^{2} \chi\left(k-k^{\prime}\right)
$$

Here $\bar{U}$ is treated as an adjustable parameter and $\chi$ is numerically calculated 224 , approximated by a phenomenological RPA-like function ${ }^{95}$ or determined experimentally from neutron scattering ${ }^{225}$ or inelastic x-ray scattering (RIXS) data 67 . These calculations find that with reasonable coupling strengths the spin-fluctuation interaction given by Eq. (18) can account for the scale of the observed transition temperatures. Note that when one speaks of pairing mediated by spin-fluctuations one is not thinking of an exchange of some boson with a sharp well defined $\omega(q)$ dispersion. Rather what is meant is that the dominant pairing interaction arises from the $S=1$ part of the particle-hole exchange contributions to $\Gamma^{p p}$. While this particle-hole exchange has some of the characteristics of a spin 1 boson, its spectral weight is spread out in momentum and frequency. This is clearly seen in the numerical calculations of $\Gamma^{p p}$ and to the extent that the spin susceptibility provides an approximation for the $\Gamma^{p p}$, it is seen directly in experimental measurements of $\chi^{\prime \prime}(q, \omega)$. Finally, it is important to keep in mind that low frequency spin fluctuations are pair breaking 226 and the optimal spin-fluctuation spectral weight for pairing occurs in a frequency range larger than twice the maximum value of the gap 227 .

This aspect of the dynamics of the pairing interaction is reflected in the rapid increase in $\Delta_{\text {Max }}(T)$ as $T$ decreases below $T_{c}$ as well as large $2 \Delta_{\text {Max }}(0) / k T_{c} \operatorname{ratios}, 227,228$. As the gap opens the low frequency pair breaking spin fluctuation spectral weight is shifted to higher energies where it contributes to the pairing, increasing the gap. The increase in the gap in turn leads to a further suppression of the low-frequency interaction spectral weight producing a positive feedback and a rapid increase of $\Delta_{\text {Max }}(T)$ as $T$ drops below $T_{c}$. Finally, at low temperatures one finds a large $2 \Delta_{\text {Max }}(0) / k T_{c}$ ratio. This is due to the altered 
spin-fluctuation spectral weight in the superconducting state which gives rise to a stronger pairing interaction than the normal state. In principle, if one could create a spin-fluctuation spectral weight in the normal state which had the same structure that it has deep in the superconducting state, one would find a significant increase in $T_{c}$.

\section{B. The bilayer Hubbard model}

Another variation of the Hubbard model, the bilayer Hubbard model, provides an interesting link between the single- and multi-orbital models. It shows how the structure of the Fermi surface or surfaces can alter the spin fluctuations and change the gap symmetry from $B_{1 g}$ ( $d$-wave) to $A_{1 g}\left(s^{ \pm}\right.$-wave). It is an example which illustrates how the spin fluctuation interaction can give rise to the different gap structures seen in the cuprate and iron-based superconductors. As shown in Fig. 25(a) in the bilayer Hubbard model, two 2D Hubbard layers are coupled by a one-electron inter-layer hopping $t_{\perp}$. For a doping near half-filling, the topological character of the non-interacting Fermi surface changes as $t_{\perp} / t$ is turned on. For example, for $\langle n\rangle=0.95$ and $t_{\perp} / t \lesssim 0.07$, the system has two electron Fermi surfaces around the origin. Then when $t_{\perp} / t \gtrsim 0.07$, the Fermi surface topology changes to one in which there is one electron and one hole-like Fermi surface as shown for $t_{\perp} / t=0.5$ and 2.0 in Fig. 25 (b) and (c), respectively. This Fermi surface structure is a simplified version of the multi-Fermi surfaces found from bandstructure calculations for the Fe-based superconductors shown in Fig. 16a.

This model, originally studied using determinant quantum Monte Carlo $25,229-231$ has also been studied using fluctuation exchange (FLEX) ${ }^{232}$, phenomenological spin fluctuation approximations ${ }^{233}, \mathrm{FRG}^{56}$ and $\mathrm{DCA}^{234}$ methods. One finds that for $t_{\perp} / t$ less than of order one, the most divergent pairfield correlations occur in the $d_{x^{2}-y^{2}}$ channel while for $t_{\perp} / t$ larger they occur in an $A_{1 g}$ channel in which the gap has one sign on the antibonding Fermi surface and the opposite sign on the bonding Fermi surface, as schematically illustrated in Fig. 25, This gap, which changes sign between the two Fermi surfaces, is an $s^{ \pm}$-like gap.

At half-filling, determinental Quantum Monte Carlo (DQMC) calculations showed that the ground state for $U=6$ had AF long-range order for $t_{\perp} / t \lesssim 2$. For larger values of $t_{\perp} / t$, the system enters a disordered valence bond phase with singlet correlations between electrons on opposite sites of the two layers. In the doped system, there is a cross-over in 

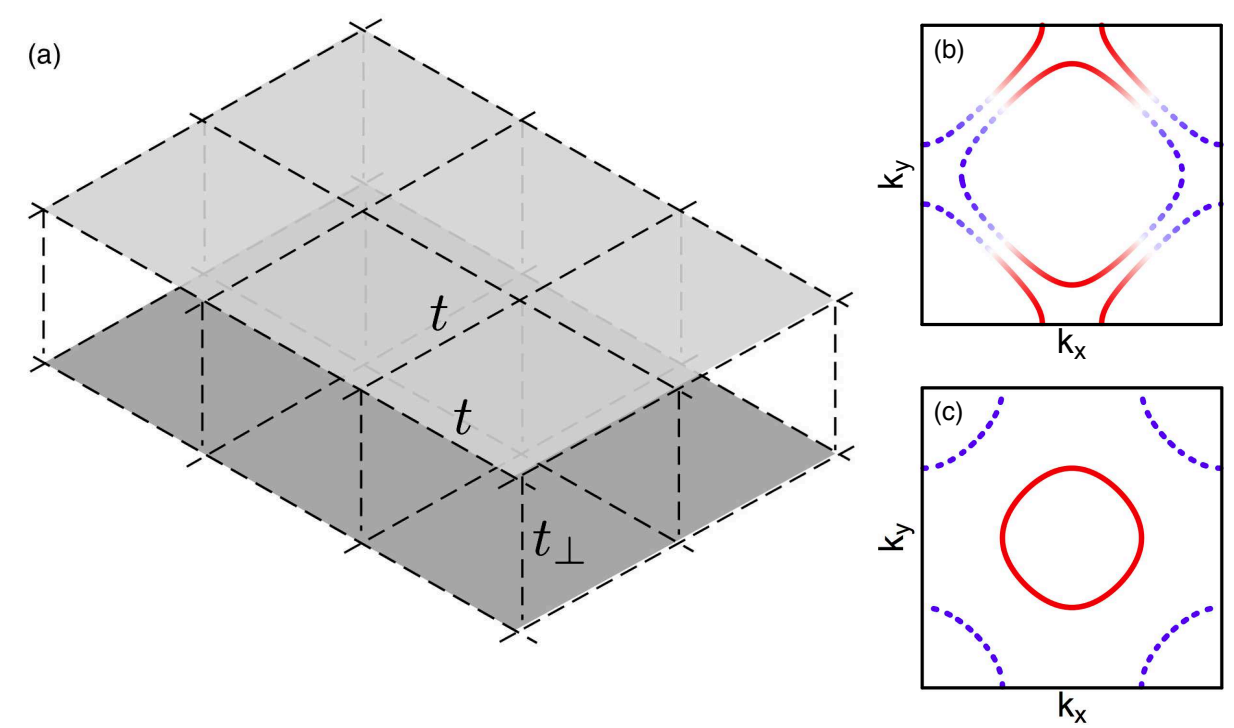

FIG. 25. The bilayer Hubbard model illustrates the close connection between the $d$ - and $s^{ \pm}$-wave states. (a) The bilayer Hubbard lattice with a near neighbor intra-layer hopping $t$ and an interlayer hopping $t_{\perp}$. (b) The bonding $\left(k_{z}=0\right)$ and antibonding $\left(k_{z}=\pi\right)$ Fermi surfaces for $t_{\perp}=0.5$ (upper) and 2.0 (lower) for a filling $\langle n\rangle=0.95$. A $d_{x^{2}-y^{2}}$ gap structure is illustrated for the $t_{\perp}=0.5$ Fermi surface and an $s^{ \pm}$gap is shown for $t_{\perp} / t=2.0$. Here, a solid (red) line denotes a positive gap and a dashed (blue) line a negative gap. The intensity of the line denotes the $d$-wave $\left(\cos k_{x}-\cos k_{y}\right)$ like variation of the gap (color online).

which the intra-layer AF fluctuations decrease and the inter-layer spin fluctuations increase as $t_{\perp} / t$ is initially increased. Then at still larger values of $t_{\perp} / t$ the low energy interlayer spin fluctuations become gapped and the superconducting pairing is suppressed.

For the two-layer system, the two pairfield susceptibilities that are of interest are given by

$$
P_{\alpha}(T)=\int_{0}^{\beta} d \tau\left\langle\Delta_{\alpha}(T) \Delta_{\alpha}^{+}(0)\right\rangle
$$

with

$$
\Delta_{x^{2}-y^{2}}=\frac{1}{\sqrt{N}} \sum_{k}\left(\cos k_{x}-\cos k_{y}\right) c_{k \uparrow}^{+} c_{-k \downarrow}^{+}
$$

and

$$
\Delta_{s^{ \pm}}=\frac{1}{\sqrt{N}} \sum_{k} \cos k_{z} c_{k \uparrow}^{+} c_{-k \downarrow}^{+} .
$$

Here for the two-layer model, $k_{z}=0$ (bonding) and $k_{z}=\pi$ (antibonding). For $U=6$ and $\langle n\rangle=0.95$, Fig. 26 shows DCA results for $P_{\alpha}(T)$ for both the $d_{x^{2}-y^{2}}$ case and the 
$s^{ \pm}$case. For $t_{\perp} / t=0.5$ where there are strong AF planar spin-fluctuations, the dominant
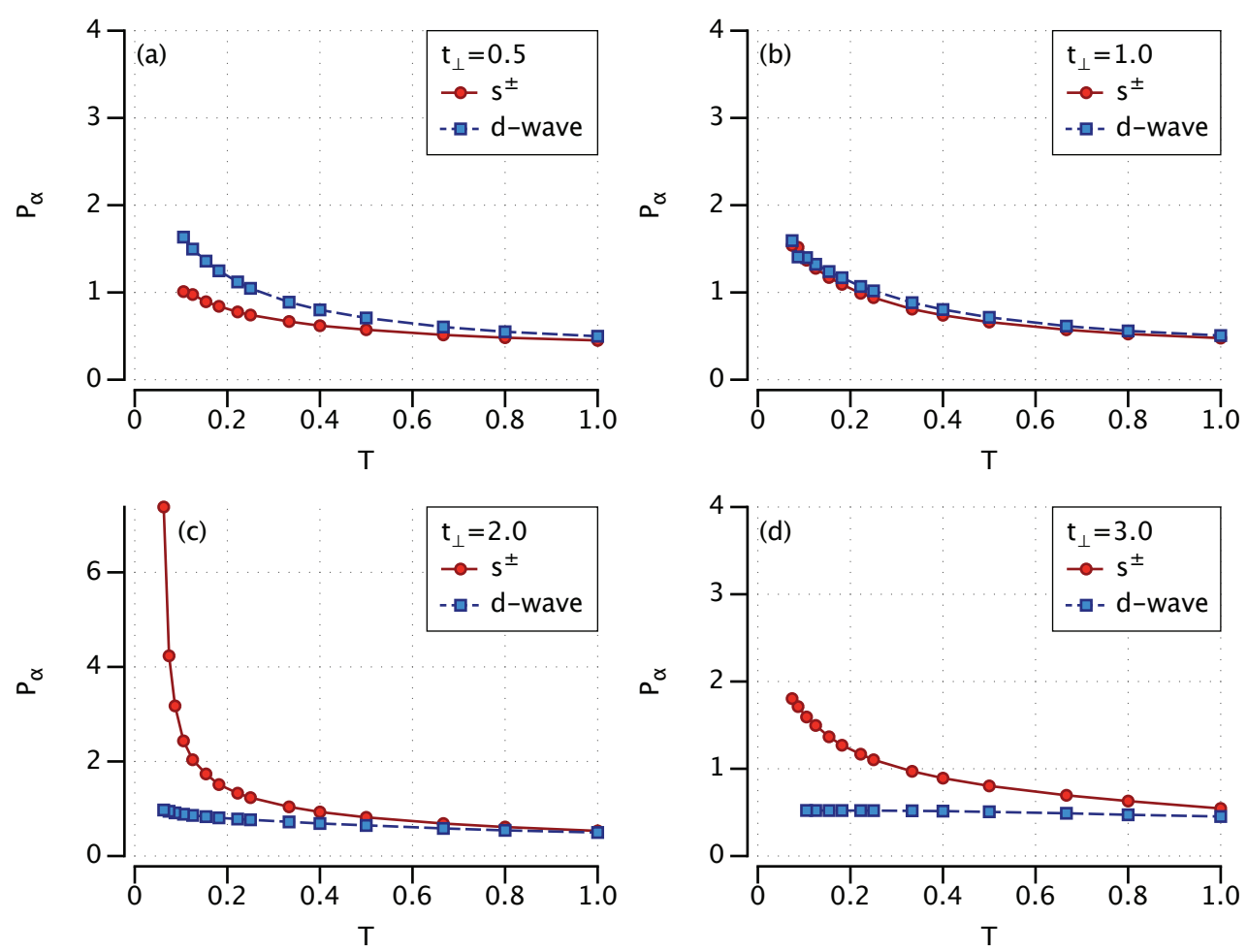

FIG. 26. The $d_{x^{2}-y^{2}}$ and $s^{ \pm}$pairfield susceptibilities $P_{\alpha}$ versus temperature $T$ for various values of the inter-layer hopping $t_{\perp}$. These DCA results were for a $(4 \times 4) \times 2$ cluster and we have set the intra-layer hopping $t=1$. One sees that as $t_{\perp} / t$ increases there is a crossover from $d_{x^{2}-y^{2}}$ pairing to $s^{ \pm}$pairing (after Maier and Scalapino 234 ).

pairing occurs in the $d_{x^{2}-y^{2}}$ channel. However, as $t_{\perp} / t$ increases, the $s^{ \pm}$response increases and for $t_{\perp} / t \gtrsim 1$, it becomes dominant with the response peaking for $t_{\perp} / t \approx 2$. At halffilling with $U / t=6, \mathrm{DQMC}$ calculations ${ }^{231}$ find a QCP for $t_{\perp} / t \approx 2$ which separates an antiferromagnetic phase from a valence bond phase ${ }^{235}$. Finally, for $t_{\perp} / t=3$ one finds that the pairing becomes weaker as the inter-layer valence bonds become stronger.

Just as the pairing interaction $\Gamma^{P P}\left(k, k^{\prime}\right)$ was analyzed for the single layer Hubbard model, one can examine how the bilayer pairing interaction is related to the underlying spin correlations of the system. A useful measure of the strength of the pairing interaction for a given channel is $\left|V_{\alpha}\right|$ given by Eq. (15). Results for $\left|V_{\alpha}\right|$ versus $t_{\perp}$ for $\alpha=d_{x^{2}-y^{2}}$ and $s^{ \pm}$ are shown in Fig. 27. Also plotted in this figure are the integrated spectral weights for the 

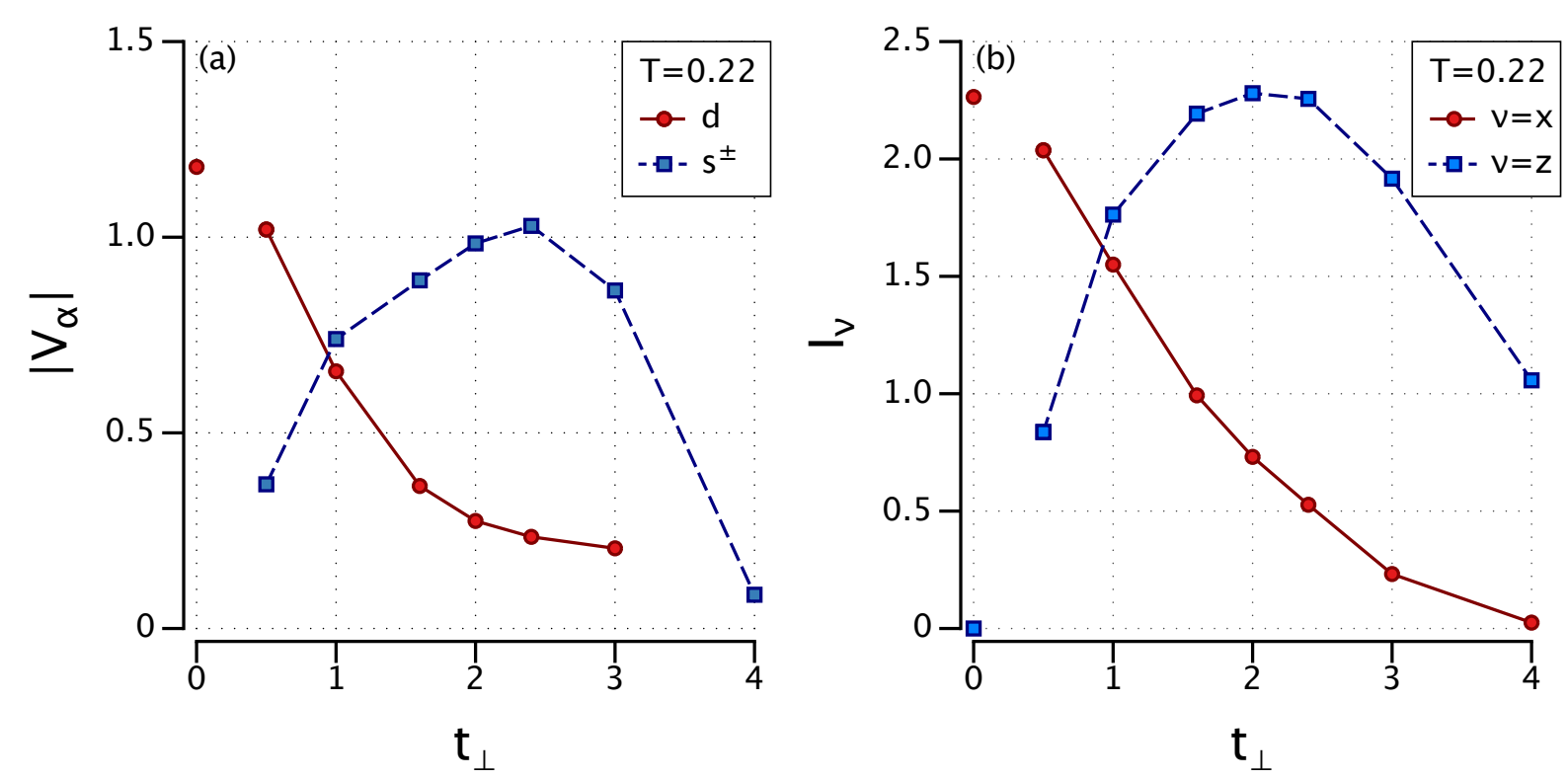

FIG. 27. The pairing interaction strength in the $d$ and $s^{ \pm}$channels reflects the spatial structure of the local spin fluctuations. (a) The strength of the pairing interactions $V_{\alpha}$ and (b) the integrated spectral weights $I_{\nu}$ versus $t_{\perp}$ for $k_{\nu}=k_{x}$ and $k_{z}$. The strength $\left|V_{\alpha}\right|$ of the pairing interaction for $d_{x^{2}-y^{2}}$ pairing is correlated with the intra-layer near neighbor spin fluctuation spectral weight, while the $s^{ \pm}$pairing strength reflects that of the inter-layer spin fluctuations (after Maier and Scalapino 234$)$.

intra- and inter-layer near-neighbor spin fluctuations

$$
I_{\nu}=\frac{1}{N} \sum_{k} \int \frac{d \omega}{\pi} \frac{\operatorname{Im} \chi(k, \omega)}{\omega} \cos k_{\nu}=\frac{1}{N} \sum_{k} \operatorname{Re} \chi(k, 0) \cos k_{\nu}
$$

with $k_{\nu}=k_{x}$ and $k_{z}$ for the intra- and inter-layer spin-fluctuation weights, respectively. In Fig. 27, one sees that the $d_{x^{2}-y^{2}}$ pairing strength is correlated with the near-neighbor planar spin fluctuations while the $s^{ \pm}$pairing strength reflects the inter-layer spin fluctuation strength.

The bilayer Hubbard model is clearly simpler than the five-orbital Fe models. However, it has the advantage that one can carry out numerical calculations and examine the relationship between the pairfield structure, the pairing interaction strengths and the spin correlations. The fact that one can change a one-electron hopping parameter $t_{\perp}$ and observe that the system evolves from a $d_{x^{2}-y^{2}}$ to an $s^{ \pm}$pairing phase provides further evidence supporting the notion of a commonality between the cuprate and Fe-based superconductors. 
A similar relationship between $d$-wave and $s^{ \pm}$pairing is seen in density matrix renormalization (DMRG) studies of a two-leg ladder ${ }^{143}$. In this case, the DMRG method has been used to study a caricature of the Fe-pnictide problem which focuses on the $d_{x z}$ orbital pair scattering process associated with the $k_{y}=0$ and $k_{y}=\pi$ states near the $\alpha_{1}$ and $\beta_{2}$ Fermi surfaces shown in Fig. 16a. These scattering processes can be described by the Hamiltonian for a 2-leg ladder
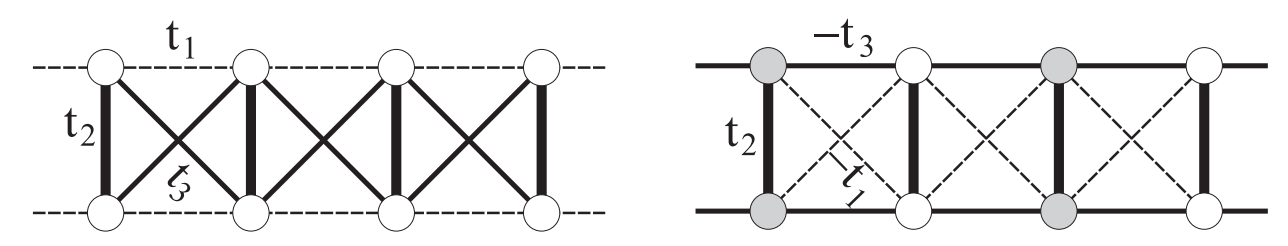

(b)
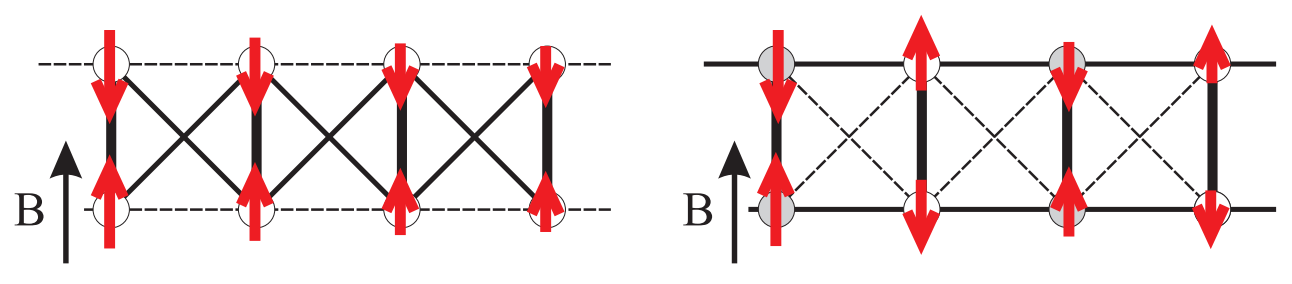

(c)
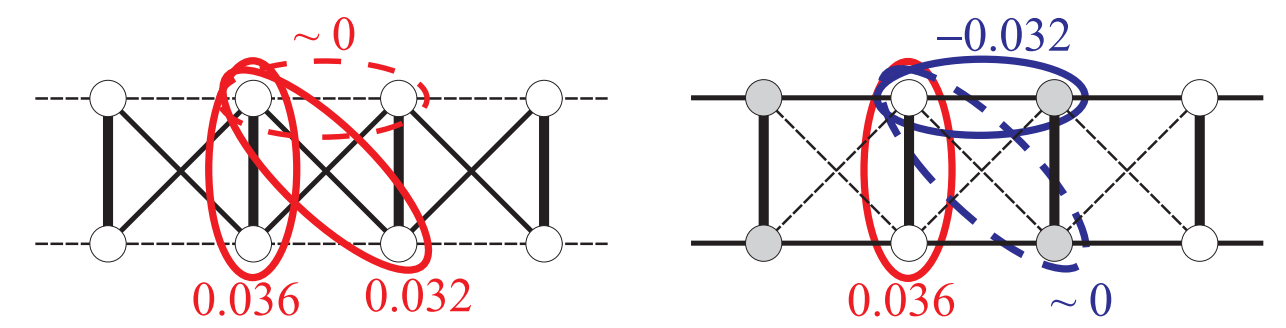

FIG. 28. An "Fe-ladder" is simply a unitary transformation of a "Cu-ladder." The left hand side shows (a) a 2-leg Fe ladder, (b) schematic illustration of the spin structure $\left\langle S^{z}\left(\ell_{x}, \ell_{y}\right)\right\rangle$ induced by applying an external magnetic field to the lower left hand site, and (c) the singlet pairfield $\left\langle\Delta_{i j}\right\rangle$ induced at a distance 10 sites removed from the end of a $32 \times 2$ ladder with a unit external pairfield applied to the end rung. On the right hand side, every other rung has been twisted by $180^{\circ}$ and the phase of the orbitals denoted by the open circles have been changed by $\pi$. As discussed in the text, this "twisted Fe-ladder" corresponds to the well-studied cuprate ladder (after Berg et al. ${ }^{143}$ ).

$$
\begin{aligned}
H=-t_{1} \sum_{i \ell \sigma} c_{i \ell \sigma}^{+} c_{i+1 \ell \sigma} & -2 t_{2} \sum_{i \sigma} c_{i 1 \sigma}^{+} c_{i 2 \sigma} \\
& -2 t_{3} \sum_{i \sigma}\left(c_{i 1 \sigma}^{+} c_{i+12 \sigma}+c_{i+12 \sigma}^{+} c_{i 1 \sigma}\right)+U \sum_{i \ell \sigma} n_{i \ell \uparrow} n_{i \ell \downarrow}
\end{aligned}
$$

with the tight binding parameters illustrated on the left-hand side of Fig. 28a. Here, $\ell=1,2$ is the leg index, there are leg $t_{1}$, rung $t_{2}$ and diagonal $t_{3}$ one-electron hopping matrix elements 
and an on-site Coulomb interaction $U$. The factors of 2 in front of $t_{2}$ and $t_{3}$ takes into account the periodic boundary conditions which have been used in the transverse direction. As discussed in Berg et al. $\stackrel{143}{ }$, the hopping parameters $t_{1}=-0.32$ and $t_{3}=-0.57$ measured in units of $t_{2}=1$, were taken to fit the Fe-pnictide (1111) DFT band structure near the $\alpha_{1}$ and $\beta_{2}$ Fermi surfaces for $k_{x}$ cuts through $k_{y}=0$ and $k_{y}=\pi$, respectively. As seen in Fig. 16a, at these points the Bloch wave functions have $d_{x z}$ character.

With $U=3$, DMRG calculations for the half-filled case with an external magnetic field applied to the first site of the lower leg gave the spin pattern shown on the left-hand side of Fig. 28b. This spin pattern has a striped-like SDW structure similar to the magnetic structure seen in the Fe-pnictides. The 2-leg system was found to have a spin gap $\Delta_{s}=0.14$ corresponding to a spin correlation length of approximately four sites. For the doped system with $\langle n\rangle=0.94$, a pairfield boundary term

$$
H_{1}=\Delta_{1}\left(P_{1}^{+}+\text {h.c. }\right)
$$

with $\Delta_{1}=1$ and

$$
P_{1}^{+}=\left(d_{11 \uparrow}^{+} d_{12 \downarrow}^{+}-d_{11 \downarrow}^{+} d_{12 \uparrow}^{+}\right)
$$

was added. This term acts as a proximity coupling to the rung at the left-hand end of the ladder. Then the expectation values of the resulting induced singlet pairfield was measured on the rung as well as the diagonal and the leg near neighbor sites at positions further down the ladder. The values of this induced pairfield 10 sites away from site $\ell=1$ are shown on the left-hand side of Fig. 28k.

This result is directly related to the 2-leg ladder cuprate model shown on the right-hand side of Fig. 28. Here, every other rung of the left-hand ladder has been twisted by $180^{\circ}$ and the phase of the $d_{x z}$-orbit has been changed by $\pi$ on each of the open sites of the twisted rungs. In this way, the rung hopping matrix element remains $t_{2}$, but the leg and diagonal hoppings are changed to $-t_{3}$ and $-t_{1}$, respectively. Then with the parameters that have been used, the dominant hoppings on this "twisted Fe-ladder" are along the legs and rungs with only a weak diagonal hopping. These are typical parameters for a cuprate ladder. Furthermore, as shown on the right-hand side of Fig. 28b and c, the resulting spin and pairfield correlations of the original Fe ladder have turned into the spin gapped $(\pi, \pi)$ antiferromagnetic and the familiar $d$-wave like pairing correlations $\frac{142}{2}$. Thus, similar to the 
2-layer Hubbard model, the 2-leg ladder illustrates the close connection that exists between the cuprates and the Fe-based materials.

\section{Multi-orbital models}

In general, for the multi-orbital models, the orbital structure of the pairing interaction is important and one introduces an orbital dependent pairing interaction $\Gamma_{\ell_{1} \ell_{2} \ell_{3} \ell_{4}}$ illustrated in Fig. 29, which describes the irreducible particle-particle scattering of electrons in orbitals
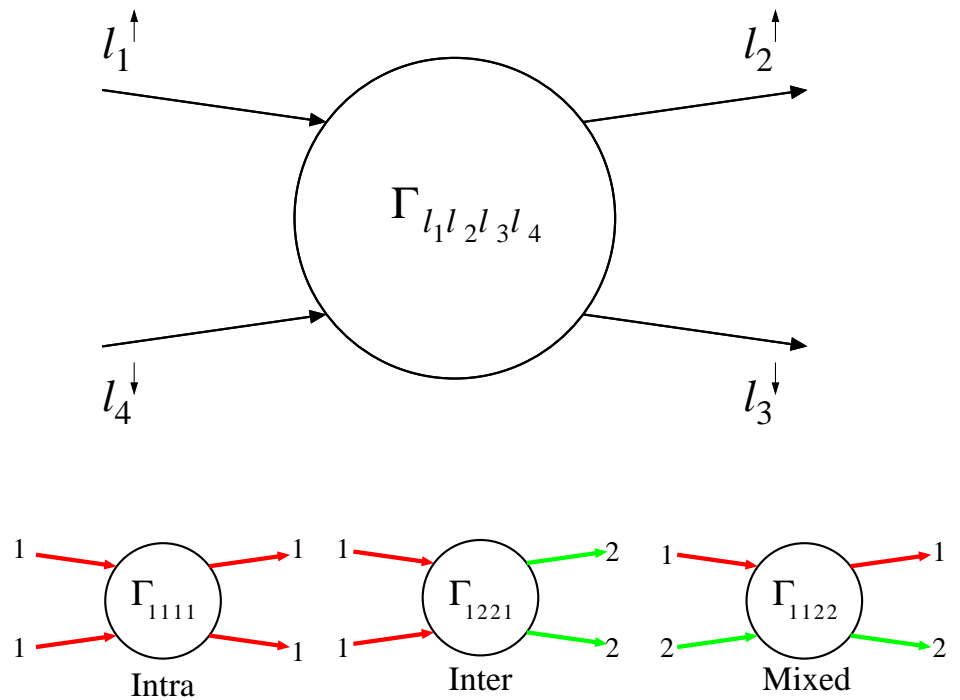

FIG. 29. The orbital dependent pairing interaction $\Gamma_{\ell_{1} \ell_{2} \ell_{3} \ell_{4}}^{\left(k, k^{\prime}\right)}$ defined in terms of orbital states $\ell_{i}$ of incoming and outgoing electrons. The lower diagrams illustrate intra-orbital, inter-orbital and mixed orbital scattering processes (after Kemper et al. ${ }^{236}$ ).

$\ell_{1}, \ell_{4}$ with momentum $k$ and $-k$ into orbitals $\ell_{2}, \ell_{3}$ with momentum $k^{\prime}$ and $-k^{\prime}$. In terms of this vertex, the effective pairing interaction for scattering a $\left(k^{\prime} \uparrow,-k^{\prime} \downarrow\right)$ pair on the $\nu_{j}$ Fermi surface to a $(k \uparrow,-k \downarrow)$ pair on the $\nu_{i}$ Fermi surface is

$$
\Gamma_{i j}\left(k, k^{\prime}\right)=\sum_{\ell_{1} \ell_{2} \ell_{3} \ell_{4}} a_{\nu_{i}}^{\ell_{2}{ }^{*}}(k) a_{\nu_{i}}^{\ell_{3}{ }^{*}}(-k) \Gamma_{\ell_{1} \ell_{2} \ell_{3} \ell_{4}}\left(k, k^{\prime}\right) a_{\nu_{j}}^{\ell_{1}}\left(k^{\prime}\right) a_{\nu_{j}}^{\ell_{4}}\left(-k^{\prime}\right)
$$

with $a_{\nu_{j}}^{\ell_{1}}(k)$ the orbital matrix element $\left\langle\nu_{j} k \mid \ell_{1}\right\rangle$ given in Eq. (9).

Besides the numerical calculations for the two-layer (effective two-obital) Hubbard model discussed above, there have been some quantum Monte Carlo 237,238 and cluster studies 220 for the three-orbital $\mathrm{CuO}_{2}$ model. These calculations show that the undoped state is a 
charge-transfer anti-ferromagnetic insulator rather than a Mott-Hubbard anti-ferromagnetic insulator. However, the anti-ferromagnetic and $d_{x^{2}-y^{2}}$-pairing correlations in the doped state of these models are remarkably similar to those found for the doped single band Hubbard model.

The main studies of the multiple-orbital models which have been carried out for the heavy fermion and Fe-based materials have been based upon weak coupling random phase $(\mathrm{RPA}) \underline{96,160,239,240}$, fluctuation-exchange (FLEX) $\underline{241}$ or functional renormalization group (FRG) methods 56 .57,242.243. Just as the Monte Carlo calculations $\frac{216}{2}$ of the four-point vertex allow one to study the interplay of the various spin, charge and pairing correlations on an equal footing as the temperature is reduced (see for example Fig. 211), the FRG provides an unbiased approach for monitoring the strength of the various scattering processes as an energy cutoff is reduced. Of course the FRG calculations are typically one-loop approximations, suitable for weaker coupled systems. Nevertheless, the FRG calculations for the multi-band Hubbard models find that spin-density-wave (SDW) scattering processes grow in strength as the renormalization energy cutoff is reduced, driving an increase in the pair scattering strength. In addition, just as for the single-band Hubbard model, strong SDW fluctuations also drive other pairing, Pomeranchuk and CDW channels. The same electrons are involved in both the spin-fluctuation and these channels.

In the RPA and FLEX approaches, the orbital dependent vertex is approximated by

$$
\begin{aligned}
\Gamma_{\ell_{1} \ell_{2} \ell_{3} \ell_{4}}\left(\mathbf{k}, \mathbf{k}^{\prime}, \omega\right)= & {\left[\frac{3}{2} U^{S} \chi_{1}^{\mathrm{RPA}}\left(\mathbf{k}-\mathbf{k}^{\prime}, \omega\right) U^{S}-\right.} \\
& \left.\frac{1}{2} U^{C} \chi_{0}^{\mathrm{RPA}}\left(\mathbf{k}-\mathbf{k}^{\prime}, \omega\right) U^{C}+\frac{1}{2}\left(U^{S}+U^{C}\right)\right]_{\ell_{3} \ell_{4} \ell_{1} \ell_{2}},
\end{aligned}
$$

with

$$
\chi_{1}^{\mathrm{RPA}}(q)=\chi^{0}(q)\left[1-U^{S} \chi^{0}(q)\right]^{-1}
$$

and

$$
\chi_{0}^{\mathrm{RPA}}(q)=\chi^{0}(q)\left[1+U^{C} \chi^{0}(q)\right]^{-1}
$$

Here the quantities $U^{S}, U^{C}$, and the one-loop susceptibility $\chi^{0}$ are represented by matrices in the orbital space. Details of this can be found in the literature ${ }^{244}$. Here we note that the basic structure of the pairing interaction is similar to Eq. (12) with

$$
\Lambda_{\mathrm{irr}} \sim \frac{1}{2}\left(U^{S}+U^{C}\right) \quad \phi_{m} \sim U^{S} \chi_{1}^{\mathrm{RPA}} U^{S} \quad \Phi_{d}=-U^{C} \chi_{0}^{\mathrm{RPA}} U^{C} .
$$


While this represents a weak coupling approximation, we know from numerical studies ${ }^{224}$ of the single band Hubbard model that by treating the interaction parameters phenomenologically, RPA and FLEX approximations can provide reasonable descriptions of the pairing interaction for intermediate coupling.

From Eq. (24) one sees that the effective pairing interaction $\Gamma_{i j}\left(k, k^{\prime}\right)$ for a multi-orbital system depends upon the number of Fermi surfaces and their shapes as well as the orbital matrix elements. In general, these matrix elements act to suppress the mixed pair orbital vertex contributions in which $\ell_{1} \neq \ell_{4}$ and $\ell_{2} \neq \ell_{3}$ (lower right hand diagram shown in Fig. 29). For spin rotational interaction parameters the dominant contributions to the pairing interaction $\Gamma_{i j}\left(k, k^{\prime}\right)$ comes from intra-orbital $\left(\ell_{1}=\ell_{2}=\ell_{3}=\ell_{4}\right)$ scattering processes with weaker contributions from the inter-orbital processes $\left(\ell_{1}=\ell_{4} \neq \ell_{2}=\ell_{3}\right)$. The number, the shape and the location of the various Fermi surfaces also play a key role in determining the strength of the pairing interaction and the structure of the gap $\Delta(k)$.

As noted by Kuroki et al. $\stackrel{239}{2}$ for the 1111 Fe material, depending upon the height of the pnictide and the doping, an additional hole Fermi surface with $d_{x y}$ orbital character may be present around the $(\pi, \pi)$ point of the unfolded Brillouin zone. Figure 30 shows the Fermi surfaces at two different fillings for a tight binding parameterization of the $1111 \mathrm{Fe}$ material. In this case, for a filling $\langle n\rangle=6.01$, there are two hole Fermi surfaces around the $\Gamma$ point and two electron Fermi surfaces around $(\pi, 0)$ and $(0, \pi)$ in the unfolded $1 \mathrm{Fe} /$ cell Brillouin zone. However, for the hole doped system with $\langle n\rangle=5.95$, an additional hole Fermi surface appears around the $(\pi, \pi)$ point. The dominant orbital weight along the Fermi surfaces are also indicated along with various intra-orbital pair scattering processes. The lefthand figure shows a pair scattering from the $\alpha_{1}$ hole Fermi surface around the $\Gamma$ point to a pair on the electron Fermi surface $\beta_{1}$ centered at $(\pi, 0)$. Here, electrons in states $k$ and $-k$ on the $\alpha_{1}$ Fermi surface are scattered to states $k^{\prime}$ and $-k^{\prime}$ on the $\beta_{1}$ Fermi surface. This process is illustrated in Fig. 30 using an extended Brillouin zone in which $-k^{\prime}$ is replaced by $-k^{\prime}+(2 \pi, 0)$. The orbital weight on both Fermi surfaces is dominantly $d_{y z}(\ell=2)$ over the regions in which there is a reasonable nesting giving rise to a peak in $\Gamma_{2222}$ for a momentum transfer $q \sim(\pi, 0)$. There are similar intra-orbital $d_{x z}$ scattering processes between $\alpha_{1}$ and the electron $\beta_{2}$ Fermi surface which give rise to a peak in $\Gamma_{1111}$ for $q \sim(0, \pi)$. These processes lead to a $\Gamma_{i j}\left(k, k^{\prime}\right)$ interaction which favors an $A_{1 g} s^{ \pm}$gap which switches sign between the $\alpha_{1}$ and the $\left(\beta_{1}, \beta_{2}\right)$ Fermi surface. However, as shown in the middle diagram of Fig. 30, there 

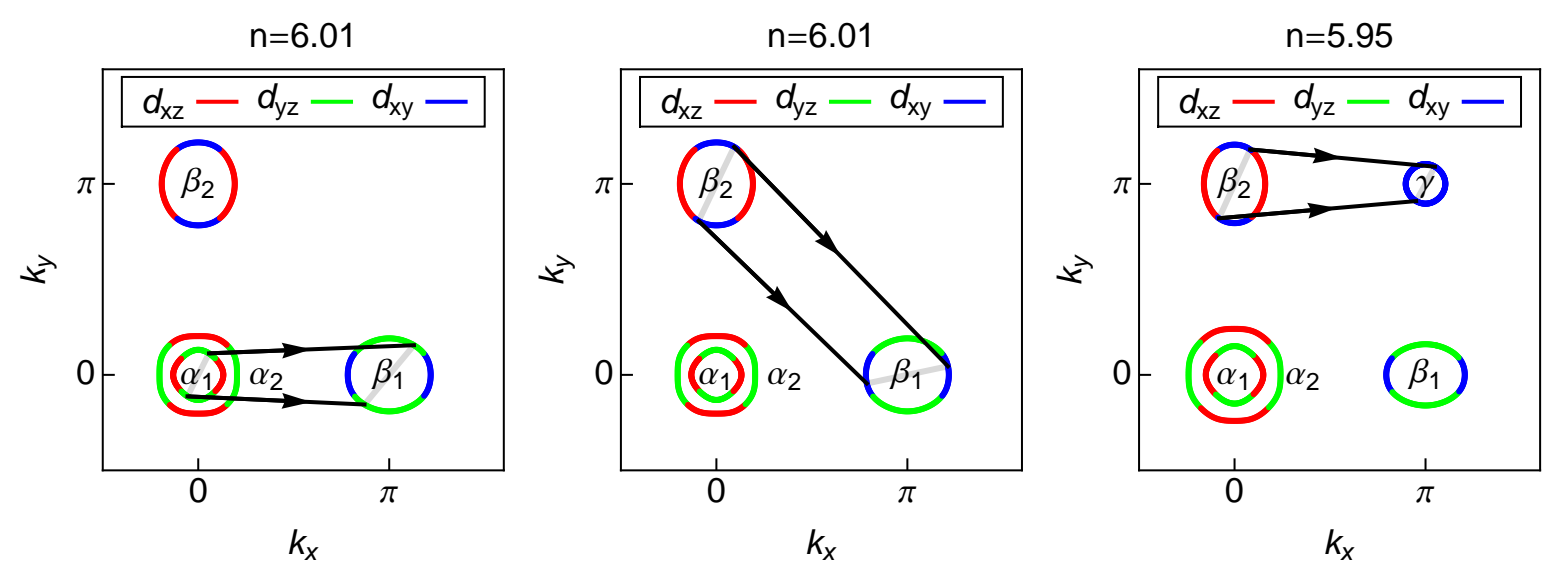

FIG. 30. For a filling $n=6.01$, the scattering of a pair from the $\alpha_{1}$ hole Fermi surface to the $\beta_{1}$ electron Fermi surface shown in the left hand figure favors pairing in which there is a sign change of the gap between $\alpha_{1}$ and $\beta_{1}$. A similar pair scattering process between $\alpha_{1}$ and $\beta_{2}$ leads to a gap which has the same sign on $\beta_{1}$ and $\beta_{2}$. However, the $\beta_{2}-\beta_{1}$ pair scattering shown in the middle figure tends to frustrate this, since they favor a gap which has opposite signs on the $\beta_{2}$ and $\beta_{1}$ Fermi surfaces. As shown in the right hand figure, for a filling $\langle n\rangle=5.95$, an additional hole pocket $\gamma$ appears and $\beta_{2}-\gamma$, as well as $\beta_{1}-\gamma$, pair scattering processes stabilize the $s^{ \pm}$gap.

are inter-orbital $d_{x z}-d_{x y}$ pair scattering processes between $\beta_{2}$ and $\beta_{1}$. These act to frustrate a uniform $s^{ \pm}$state. This same behavior is seen in the FRNG calculations $\frac{56,245}{2}$. In addition, unless the Fermi surface areas weighted by $v_{F}^{-1}(k)$ are such that the electron and hole regions exactly balance, the short range Coulomb interaction can be reduced by an anisotropic $A_{1 g}$ gap. As a consequence, for a filling $\langle n\rangle=6.01$ and a typical set of interaction parameters, one finds the $A_{1 g}$ gap structure shown on the left of Fig. 31 and as the blue curve in Fig. 32 , Here the gap has nodes on the $\beta$ electron Fermi surfaces. The possibility of such accidental nodes in the $A_{1 g}$ state is consistent with the linear low temperature $T$ dependence seen in the penetration depth of $\mathrm{LaFePO}^{246}$.

The gap $\Delta(k)$ for $\langle n\rangle=6.01$ and $\langle n\rangle=5.95$ is shown in Fig. 31. For $\langle n\rangle=6.01$ the $(\pi, \pi)$ Fermi surface is absent while for a doping $\langle n\rangle=5.95$, there is an additional hole Fermi surface around the $(\pi, \pi)$ point of the $1 \mathrm{Fe} /$ cell Brillouin zone. In this latter case, intra-orbital $d_{x y}$ pair scattering processes like the one shown in the right panel of Fig. 30 favor a more uniform $s^{ \pm} A_{1 g}$ state and as shown on the right hand side of Fig. 31 and the red curve in Fig. 32, the nodes on the $\beta$ Fermi surfaces are lifted. In addition the overall pairing 

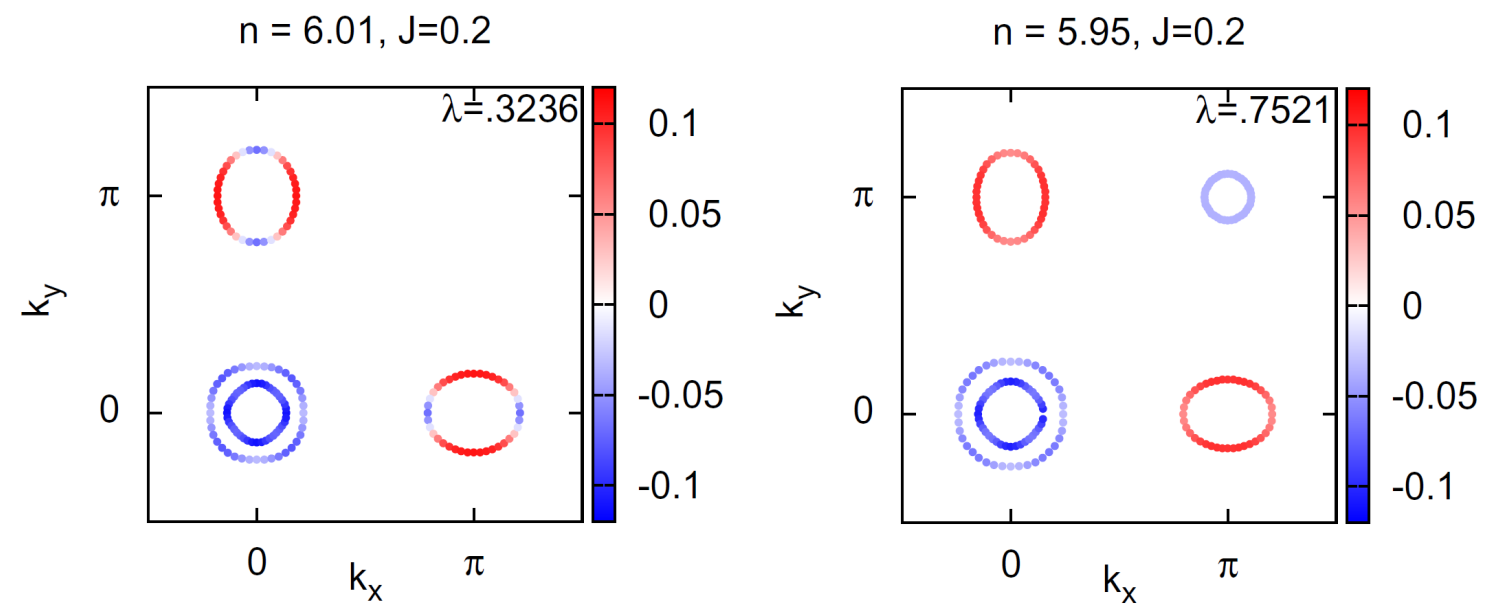

FIG. 31. The gap eigenfunctions $g(k)$ for a spin rotationally invariant parameter set $\bar{U}=1.3$, $\bar{U}^{\prime}=0.9, \bar{J}=\bar{J}^{\prime}=0.2$, for dopings $n=6.01$ (left) and $n=5.95$ (right). Here, one sees how the $s^{ \pm}$ gap is stabilized by the $\beta_{1}-\gamma$ and $\beta_{2}-\gamma$ pair scattering processes shown in the right hand portion of Fig. 30 (after Kemper et al $\stackrel{236}{ }$ ).

strength is larger when the extra hole $(\pi, \pi)$ Fermi surface is present. In similar calculations in which the bandstructure parameters were changed so that the $(\pi, \pi)$ Fermi surface had dominant $d_{3 z^{2}-r^{2}}$ weight, the nodes of the gap were not lifted. Thus the orbital weights as well as the Fermi surface topology play an important role in determining the gap structure as well as $T_{c}^{236,239,243,245,247}$.

\section{SUMMARY AND OUTLOOK}

Here it has been proposed that the interaction which is responsible for pairing in some families of heavy fermion materials, the $115 \mathrm{Pu}$ actinides, the high $T_{c}$ cuprates and the Fe-based superconductors arises from the exchange of spin-fluctuations. Just as different materials ranging from $\mathrm{Hg}$ and $\mathrm{Pb}$ to $\mathrm{Nb}_{3} \mathrm{Sn}$ and $\mathrm{MgB}_{2}$ have a phonon mediated pairing interaction, the suggestion is that this class of unconventional superconducting materials, though clearly different from each other, share a common pairing mechanism. As noted earlier, one should also include the organic Bechgaard salts ${ }^{3-6}$ in this group. ${ }^{248}$ Looking back with this perspective, one would say that this class of antiferromagnetic spin-fluctuation mediated superconductors began with the seminal discoveries of superconductivity in the heavy fermion material $\mathrm{CeCu}_{2} \mathrm{Si}_{2}$ by Steglich et al. $\stackrel{249}{2}$ and in the organic material (TMTSF) ${ }_{2} \mathrm{PF}_{6}$ 


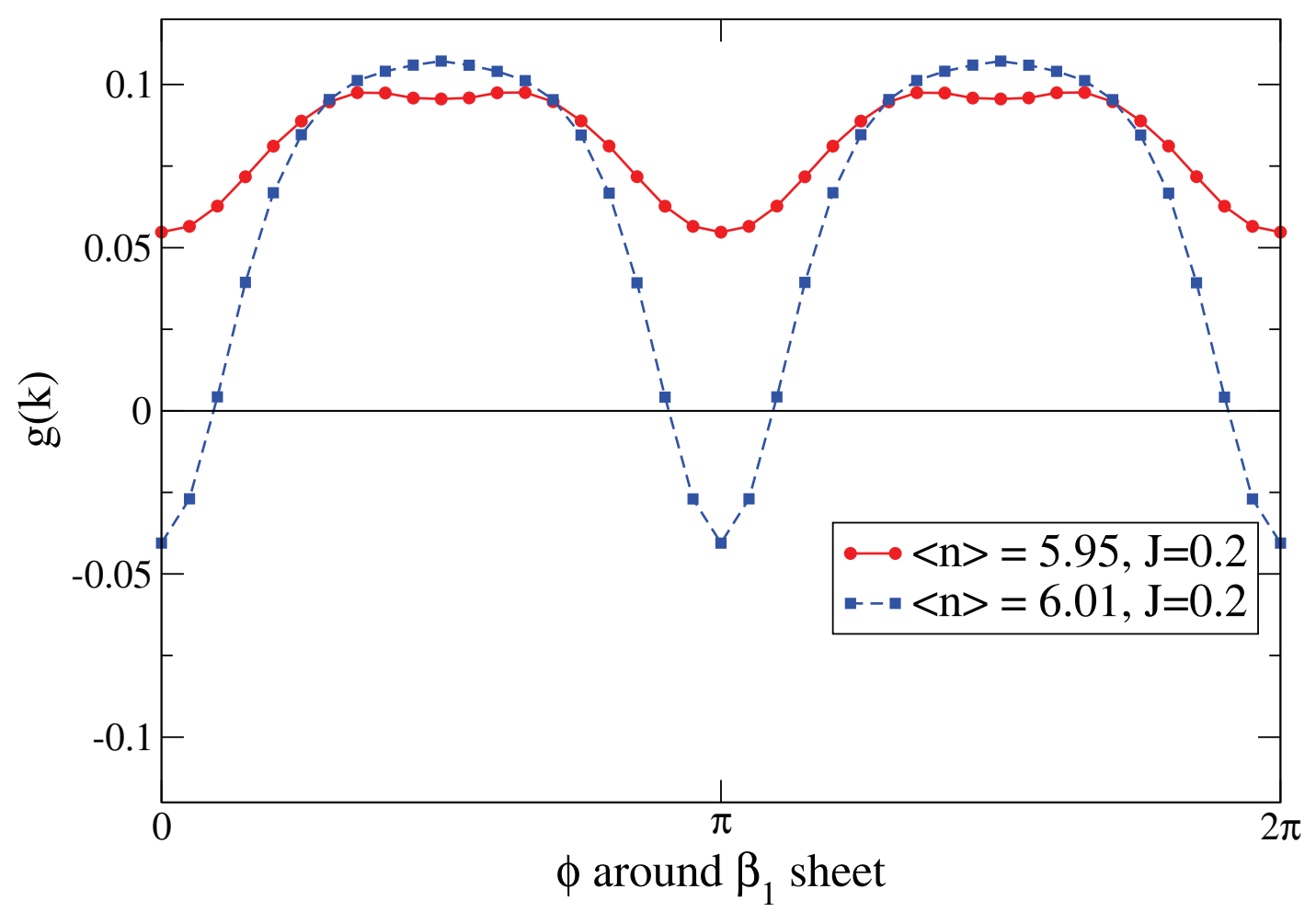

FIG. 32. The gap function $g(k)$ on the $\beta_{1}$ pocket for $n=5.95$ solid (red) and $n=6.01$ dashed (blue) from Fig. 31. Here the angle $\phi$ is measured from the $k_{x}$-axis (after Kemper et al. $\stackrel{236}{ }$ ).

by Jérome et al. $\stackrel{250}{ }$.

Theoretical proposals that spin-fluctuations near a spin-density-wave instability could give rise to unconventional pairing in some organic Bechgaard salts and some heavy fermion materials were made in 1986 (Emery $\stackrel{251}{ }$, Cyrot $\stackrel{252}{ }$, Miyake et al. .93 , Scalapino et al. $\underline{94}$ ). Then, following the discovery of the cuprate superconductors various suggestions were made to also include the cuprates in this group $103,253,254$. However, while the antiferromagnetism and $d-$ wave superconductivity appeared in close proximity in the phase diagrams of the electron doped cuprates, in the hole doped cuprates, a pseudogap phase appears adjacent to the superconducting phase. Furthermore, the undoped cuprates are antiferromagnetic chargetransfer Mott insulators. Thus there were arguments made that superconductivity in the high $T_{c}$ cuprates arose from a different underlying mechanism, and that it was inappropriate 
to speak of a spin-fluctuation pairing glue $\frac{255}{2}$. Now the question of whether there is a pairing glue is basically a question regarding the dynamics of the pairing interaction 256 . As discussed in Sec. IV, numerical calculations of the pairing interaction for the Hubbard model provide evidence which supports the view that its dynamics dominantly reflects that of the dynamic spin susceptibility $\underline{218}-\underline{220}$. Thus there is pairing glue in the Hubbard models and the question becomes "Should one speak of a spin-fluctuation pairing glue for this class of real materials?"

The discovery of the Fe-based superconductors ${ }^{22.23}$ provided renewed support for the idea that indeed there exists a class of materials in which superconductivity does not arise from the traditional phonon exchange mechanism 257 . In addition, as noted in Sec. II, a variety of measurements show that antiferromagnetic spin-density-wave-like fluctuations are ubiquitous in these materials and are the primary excitations which scatter the electrons. Now in principle, one would like to determine the $k$ and $\omega$ dependence of the normal and anomalous (gap) self-energies and from these infer the structure and origin of the pairing interaction. In particular, the $k$-dependence of the gap on the multi-Fermi surfaces of the Fe-based superconductors can provide a more detailed probe of the $k$-dependence of the pairing interaction providing a test of different pairing mechanisms. For example, the spinfluctuation theory finds that there can be a near-degeneracy between an anisotropic signchanging $s$-wave $\left(A_{1 g}\right)$ state and a $d_{x^{2}-y^{2}}\left(B_{1 g}\right)$ state due to the near nesting of Fermi surface sheets $\frac{96}{258}$. This is also clearly seen in the DCA results for the bilayer model discussed in Sec. IV, Thus the $k$-dependence of the gap on the multi-Fermi surfaces of the Fe-based superconductors can provide a test of the theory. In addition, as dicussed in Sec. IV] there are a number of experiments which are exploring the $\omega$ dependence of the gap. The recent progress in material quality, the increase in the frequency and momentum resolution of ARPES, neutron scattering and RIXS, along with tunneling and STMS hold the promise of providing the kind of detailed information that will be needed. There will also be support for these ideas if they can provide guidance in the search for new and possibly higher $T_{c}$ superconductors. This review concludes by summarizing some of the ideas which have been discussed that may help in this search.

The numerical calculations for the doped single band Hubbard model with a near neighbor hopping $t$ and an onsite Coulomb interaction $U$, show that $T_{c}$ is maximized for $U$ of order the bandwidth $8 t$. As $U$ increases beyond the bandwidth, the characteristic energy of the spin fluctuations is suppressed and $T_{c}$ decreases. In addition, $T_{c}$ is found to decrease in 
the underdoped regime. Here, the superfluid stiffness tends to zero as the Mott state is approached 259 . In addition, there is the reduction of the quasi-particle weight due to the Mott correlations which suppress the intrinsic pairfield susceptibility $P_{0 d}$. Thus optimal superconductivity is obtained by doping the single layer Hubbard model away from halffilling. In the doped bilayer case, $T_{c}$ is enhanced when $t_{\perp} / t$ is increased and in this way the system is again moved away from the Mott regime to a semi-metallic state. Thus optimal superconductivity in these models is expected to be found at intermediate coupling away from the Mott regime. In this regime, the fluctuation-exchange (FLEX) approximation ${ }^{100}$ gives results in reasonable agreement with the numerical calculations and it has been used to address further issues.

The phase diagram obtained for a two-dimensional Hubbard model with $U / t=4$ using FLEX is shown in Fig. 33. Here one sees that as the system is doped, the SDW antifer-

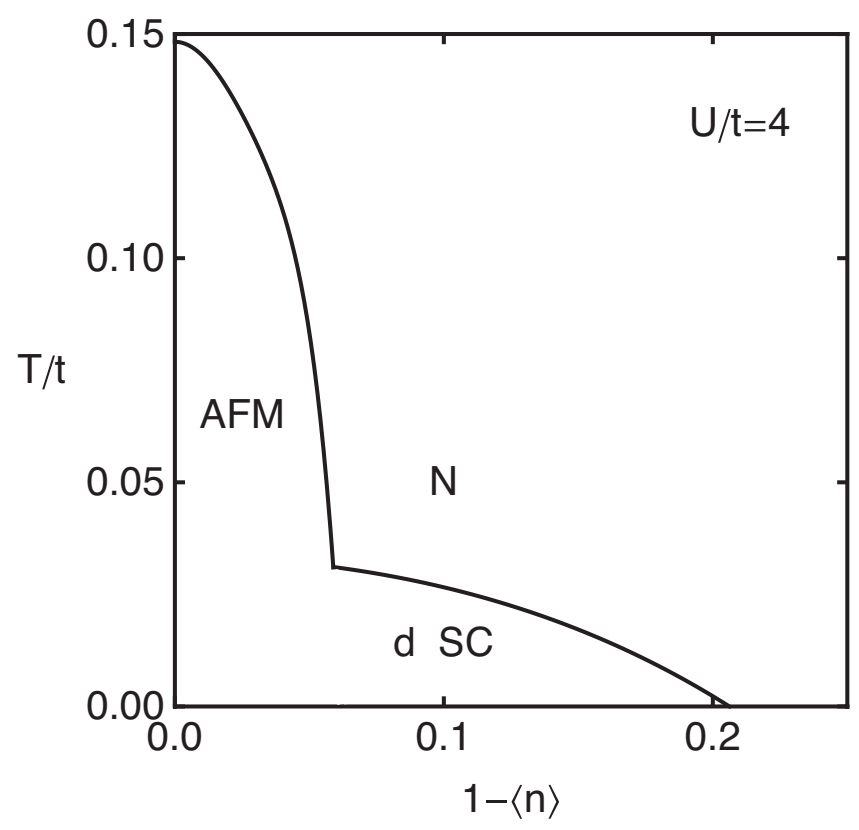

FIG. 33. Phase diagram for a two-dimensional Hubbard model with $U / t=4$ calculated within the fluctuation-exchange approximation. As the system is doped away from half-filling, the Néel temperature is suppressed and a $d_{x^{2}-y^{2}}$ superconducting phase appears (after Bickers et al. ${ }^{100}$ ).

romagnetic phase is suppressed and $d_{x^{2}-y^{2}}$-wave superconductivity appears. As discussed by Vorontsov et al. $\underline{\underline{47}}$ and Fernandes et al. $\underline{45}$ there can be a coexistence region near the intersection of the antiferromagnetic and superconducting transitions. As the doping in- 
creases, in the absence of the superconducting transition, the antiferromagnetic transition is suppressed towards $T=0$ giving rise to a quantum critical point (QCP) ${ }^{73,235}$. The shape of the phase boundaries as well as the temperature dependence of the transport properties reflect the antiferromagnetic spin fluctuations associated with the $\mathrm{QCP} 68,260,261$. The precise role of the QCP remains under study. Within the framework of FLEX calculations, the characteristic antiferromagnetic energy at zero doping $T_{N}(x=0)$ is large compared with $T_{c}$. In this case, to optimize $T_{c}$ one changes the doping $x$ so as to reduce the frequency of the antiferromagnetic fluctuations to some multiple of $T_{c}$ in order to optimize the pairing. Since $T_{N} \gg T_{c}$, this means that one will indeed have to tune the doping $x$ close to the critical concentration $x_{c}$ where $T_{N}\left(x_{c}\right)$ would vanish in the absence of superconductivity.

With a near neighbor hopping $t$, a nominal filling $\langle n\rangle \sim 0.85$ and $U / t$ fixed, the size of the transition temperature $T_{c}$ scales with the energy scale $t$. In this framework then, the range of $T_{c}$ values found between the heavy fermion materials and the cuprates is seen as a reflection of their electronic energy scales. This notion, that the variation of $T_{c}$ depended on a basic electronic energy scale of the material, was considered within a fluctuation-exchange treatment of the single-band Hubbard model by Moriya and Ueda $\frac{103}{}$ who related this scale to a spin fluctuation energy $T_{\mathrm{SF}}$. In their approach $T_{\mathrm{SF}} \simeq 1.25 \times 10^{4} / \gamma$ with the specific heat $\gamma$ measured in $m J / m o l K^{2}$ and the spin-fluctuation cut-off wave vector taken to be of order the zone boundary wave vector. Based on these results, they proposed a unified picture in which $T_{c}$ varied as $T_{\mathrm{SF}}$. Alternatively, Uemura ${ }^{262}$ has used an effective Fermi energy obtained from the penetration depth in place of $T_{\mathrm{SF}}$. The basic idea is similar to what one finds in the Hubbard model where with $U$ and $\langle n\rangle$ optimized, $T_{c}$ is set by the energy scale $t$. Figure 34 shows this type of Moriya-Ueda plot with the addition of the 115 $\mathrm{Pu}$ actinides. Curro et al. ${ }^{263}$ noted that the $115 \mathrm{Pu}$ actinides could be added to this group of materials providing a natural bridge between the heavy fermions and the high $T_{c}$ cuprates. In this case, the larger $T_{c}$ values of the $115 \mathrm{Pu}$ compounds relative to the $115 \mathrm{Ce}$ systems is a reflection of the larger hybridization among the $5 f$ electrons of the $115 \mathrm{Pu}$ compounds and hence to a larger value of the basic energy scale ${ }^{37,264}$. In a similar way, the unit cell volume of $\mathrm{PuCoIn}_{5}$ is nearly $28 \%$ larger than that of $\mathrm{PuCoGa}_{5} \frac{15}{5}$, leading to a weaker hybridization and a reduced $T_{c}$.

In addition to the intermediate coupling requirement and the size of a basic energy scale, the topology of the Fermi surface as well as the orbital weights on the Fermi surface play 


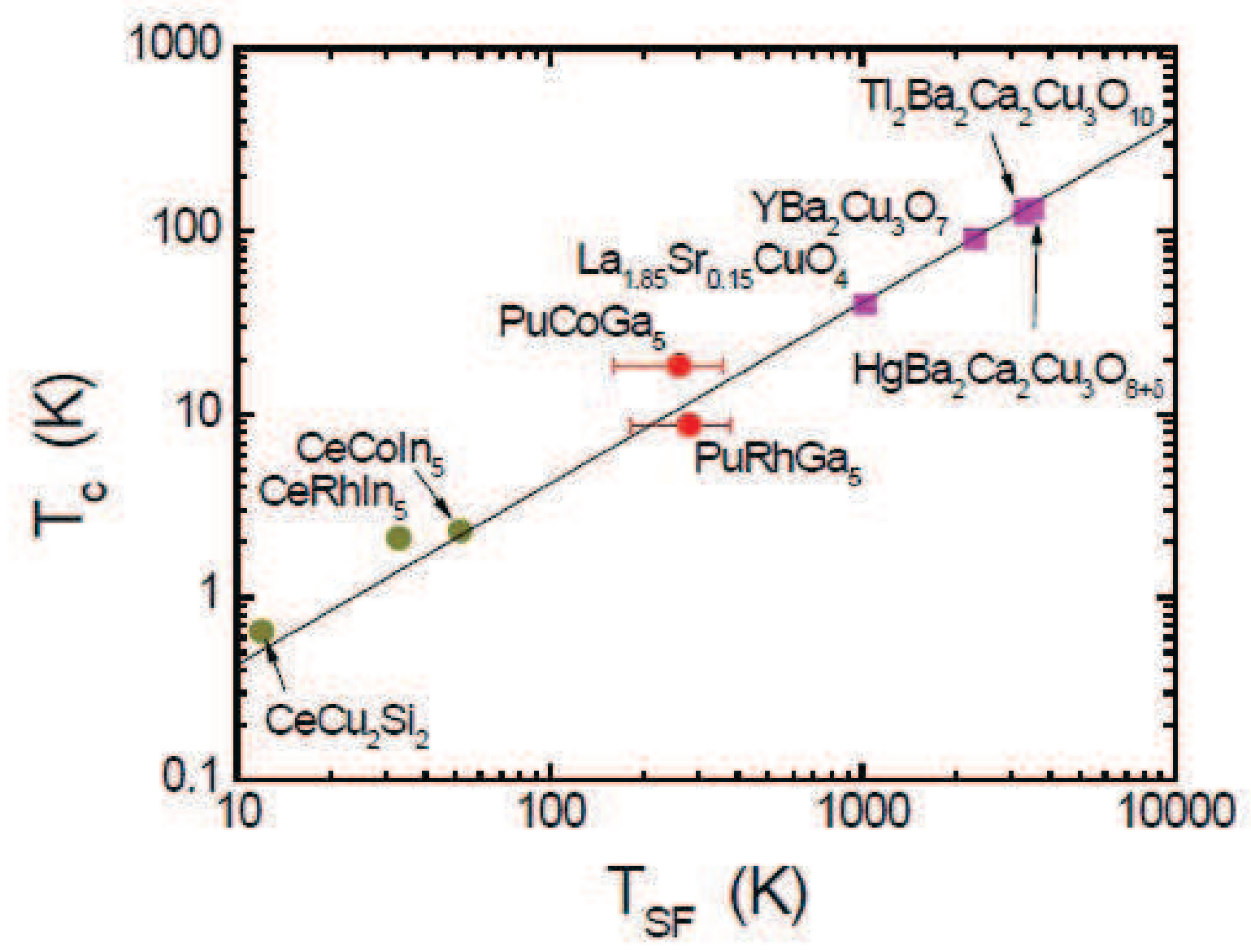

FIG. 34. A Moriya-Ueda like plot of the temperatures of various unconventional superconductors plotted against $T_{\mathrm{SF}}$, a characteristic temperature indicating the energy spread of the wave vectordependent part of the spin-fluctuations (after Curro et al. 263 ).

an important role in determining $T_{c}$. As noted in Sec. IV, the lattice structure and/or doping can alter the number of Fermi surfaces of the Fe-pnictide materials. Kuroki et al. $\underline{239}$ have suggested that the pnictogen height $h_{\mathrm{pn}}$ above the Fe layer controls the appearance of a $d_{x y}$ hole pocket around the $(\pi, \pi)$ point of the unfolded $1 \mathrm{Fe} /$ cell Brillouin zone. They noted that when $h_{\mathrm{pn}}$ is such that the pnictogen ions form a nearly regular tetrahedron as in $\mathrm{NdFeAsO}\left(T_{c} \sim 50 \mathrm{~K}\right)$, the nearest-neighbor hopping for the $d_{x y}$ orbital (here $x, y, z$ refer to the single $\mathrm{Fe} /$ cell lattice) decreases and an additional $d_{x y}(\pi, \pi)$ hole pocket appears. Spin fluctuation mediated scattering of pairs between this pocket and the $d_{x y}$ regions of the $\beta_{1}$ and $\beta_{2}$ electron pockets at $(\pi, 0)$ and $(0, \pi)$ lead to a nodeless $A_{1 g}$ gap. However for LaFePO, the pnictide $\mathrm{P}$ is closer to the Fe plane and the Fe-pnictogen-Fe angle is considerably larger than that of a regular tetrahedron. In this case, the $(\pi, \pi)$ hole Fermi pocket is absent and as 
discussed in Sec. IV, the spin-fluctuation and the Coulomb interaction favor a nodal $A_{1 g}$ gap which has a lower $T_{c}$. Similarly, as discussed by Usui and Kuroki, for the 1111 Fe-pnictide structure, if the Fe-pnictogen-Fe angle becomes small relative to the regular tetrahedron, the $\alpha_{1}$ hole Fermi surface disappears and $T_{c}$ decreases.

Multi-orbital effects also appear to play a role in the relative $T_{c}$ values of the cuprates. Based on electronic structure calculations, Pavarini et al. $\underline{266}$ observed that the $T_{c}$ of the hole cuprate materials was related to the energy of a hybrid orbital formed between the apicaloxygen and the planar coopers. They noted that the axial orbital controlled the range $r$ of the intralayer hoppings and $T_{c}$ was found to increase with $r$. This range parameter $r$ was found to increase as the apical $\mathrm{O}$ moved away from the $\mathrm{CuO}_{2}$ plane. It was also suggested by Ohta et al.267 that $T_{c}$ of the hole doped cuprates was correlated with the energy difference between the apical $\mathrm{O} p_{z}$ and planar $\mathrm{O} p_{\sigma}$ orbitals. Recently, Sakakibara et al. $\underline{268}$ argued that these correlations could be understood in terms of a two orbital Hubbard model that included in addition to the $d_{x^{2}-y^{2}} \mathrm{Cu}$ orbit of the standard one-band Hubbard model an additional $d_{3 z^{2}-r^{2}}$ orbit. They focused on the question of why the superconducting transition temperature of the single layer $\mathrm{HgBa}_{2} \mathrm{CuO}_{4+\delta}\left(T_{c} \sim 90 \mathrm{~K}\right)$ is significantly higher than the single layer $\mathrm{La}_{2-x}(\mathrm{Sr} / \mathrm{Ba})_{x} \mathrm{CuO}_{4}\left(T_{c} \sim 40 K\right)$. Within the fluctuation-exchange approximation, they found that the eigenvalue of the Bethe-Salpeter equation (13) decreased when the $d_{x^{2}-y^{2}}$ orbital weight on the Fermi surface was reduced by an admixture of $d_{3 z^{2}-r^{2}}$ orbital weight. They noted that the $d_{3 z^{2}-r^{2}}$ orbital weight was controlled by the height of the apex oxygen and the Madelung potential difference between the planar and apical oxygens, in agreement with the earlier proposals. The reduction of the pairing strength arising from the admixture of other orbitals was also found in FRG calculations $\stackrel{243}{ }$. Similarly, the level splitting of a two orbital model of the $115 \mathrm{CeCoIn}_{5}$ and $\mathrm{CeRhIn}_{5}$ heavy fermion materials has also been used to discuss their $T_{c}$ differences $\underline{244}$. Here the $\Gamma_{\alpha}$ levels are split by the tetragonal crystal field and $T_{c}$ was found to increase with this splitting.

With respect to guidance in the search for new and possibly higher temperature superconductors, these results suggest that one is looking for materials containing quasi 2D layers of $3 \mathrm{~d}$ ions. One wants magnetic ions to boost the amplitude of the spin fluctuations and $3 \mathrm{~d}$ ions rather than $4 \mathrm{~d}$ or $5 \mathrm{~d}$ ions which have a smaller effective Coulomb interaction or $4 f$ or $5 f$ ions which have a narrower bandwidth and hence a smaller basic energy scale. One wants $2 \mathrm{D}$ layers so that the antiferromagnetic order is suppressed and the spectral weight 
of the spin fluctuations is in a frequency range several times the maximum gap where it is most effective in pairing. In addition, in $2 \mathrm{D}$ it is possible that a larger fraction of a cylindrical Fermi surface or surfaces can simultaneously be "optimized" with respect to the pairing 269 . The Fe-pnictides suggest a further optimization scheme in which adding an additional Fermi surface 239,265 with a particular orbital character allows for additional scattering processes leading to a higher $T_{c}$. Here, as discussed not only the presence of the additional Fermi surface is important but it must have the right orbital character. It is generally better with respect to both the pairing strength and $T_{c}$ to have a nodeless gap instead of a nodal gap, and therefore a multi-Fermi surface system is favored.

Finally, it may be possible to find structures which have spatial or dynamic properties which enhance $T_{c}$. Here one has the idea of optimal inhomogeneity in which a composite material consisting of a "pairing region" with a large gap scale is coupled to a "metallic region" which provides phase stiffness ${ }^{43}$. Examples of this range from weakly coupled twoleg ladder systems ${ }^{270}$ which could have a period 4 bond-centered stripe structure to layered materials 271 . As noted in Sec. IV, one might also wonder whether it might be possible to alter the dynamic structure of the spin-fluctuation spectrum in a manner that would increase $T_{c}$. Here the idea would be to move the low frequency spin fluctuations to higher frequency in the normal system so as to obtain the increase in the pairing strength that is ultimately available in the usual superconducting state in which the pairing gap has opened. Here of course one would need to do this without suppressing the intrinsic pairfield susceptibility.

So we will end this review as it began by noting that while, in principle, the momentum and frequency dependence of the superconducting gap can provide a fingerprint to identify the pairing interaction, it will be the material record ${ }^{1}$ that will tell us whether these ideas proved useful in providing guidance in the search for new superconductors.

\section{Appendix A: The Structure of Two Pairing Interactions}

As discussed in Sec. IV, the Coulomb interaction $U$ gives rise to short-range antiferromagnetic spin fluctuations which produce a pairing interaction that is non-local in space and retarded. In particular, as illustrated in Fig. 19, this pairing interaction is repulsive for two electrons on the same site but attractive if the electrons are on near neighbor sites. Thus if the paired electrons are spatially correlated so as to avoid occupying the same site, they 
can take advantage of the non-local near-neighbor attractive part of the interaction. This spatial non-local nature of the Hubbard model pairing interaction has an analogy with the temporal, retarded nature of the familiar electron-phonon screened Coulomb pairing interaction. In this appendix, the structure of the traditional electron-phonon screened Coulomb interaction will be compared with the structure of the spin-fluctuation interaction. Here to ease the notation, we will drop the superscript index $p p$ and $\Gamma$ will denote the irreducible particle-particle vertex which we will call the pairing vertex.

\section{The Electron-phonon Screened Coulomb Pairing Interaction}

To begin, consider the well-known approximation of the pairing vertex for the traditional electron-phonon screened Coulomb model 272 illustrated in Fig. 35

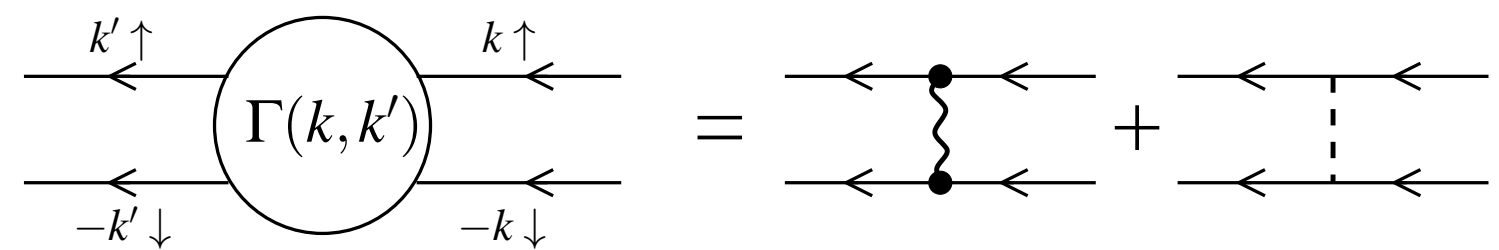

FIG. 35. The traditional approximation to the pairing vertex $\Gamma\left(k, k^{\prime}\right)$ for the electron-phonon screened Coulomb model. Here the wavy line represents the dressed phonon propagator, the dots the dressed electron-phonon couplings and the dashed line a screened Coulomb interaction.

$$
\Gamma\left(q, \omega_{m}\right)=-\frac{\left|g_{q}\right|^{2} 2 \omega_{q}}{\omega_{m}^{2}+\omega_{q}^{2}}+\frac{4 \pi e^{2}}{q^{2}+\kappa^{2}} .
$$

Here $q=k^{\prime}-k$ and $\omega_{m}=\omega_{n^{\prime}}-\omega_{n}$ are the momentum and Matsubara energy transferred in the scattering, and we have omitted a sum over the phonon polarizations. The first term in Eq. (A1) is the phonon-exchange term with $g_{q}$ the electron-phonon coupling constant and $\omega_{q}$ the phonon energy. The second term is the screened Coulomb interaction with $\kappa$ the Thomas-Fermi screening wavevector. This form of the vertex, with the phonon frequencies and the electron-phonon coupling determined from bandstructure and linear response calculations, has provided a useful approximation for the conventional superconductors 273 . In this case, as discussed by Migdal and Eliashberg, vertex corrections to the electronphonon term are of order the ratio of the Debye energy to the Fermi energy and can be 
neglected. Furthermore, for materials with negligible magnetic correlations, the screened Coulomb term (which ultimately is replaced by a Coulomb pseudopotential $\mu^{* 276,277}$ ) has proved an adequate representation of the Coulomb interaction.

Continuing with the traditional approach, we note that the important pair scattering processes take place on the Fermi surface and the dominant part of the phase space is associated with large momentum transfers of order $2 p_{F}$. For these large momentum transfers, $g_{q}$ and $\omega_{q}$ are slowly varying functions of $q$, as is the screened Coulomb interaction. This means that the interaction is local in space but retarded in time. Averaging the momentum transfer over the Fermi surface, and taking an Einstein spectrum $\omega_{q}=\Omega$ for the phonons, the pairing interaction becomes

$$
\Gamma\left(\omega_{m}\right) \approx-\frac{2|g|^{2} \Omega}{\omega_{m}^{2}+\Omega^{2}}+V_{c}
$$

Here,

$$
V_{c}=\frac{\left\langle\frac{4 \pi e^{2}}{q^{2}+\kappa^{2}}\right\rangle_{\mathrm{FS}}}{N(0)}
$$

with $N(0)$ the single spin density of states at the Fermi surface.

A plot of $\Gamma\left(\omega_{m}\right)$ is shown in Fig. 36a for a typical set of parameters for which $-\frac{2|g|^{2}}{\Omega}+V_{c}>$ 0 . In this case, the effective pairing interaction is positive for all Matsubara frequencies $\omega_{m}$ and might naïvely appear to be repulsive. $\stackrel{278}{2}$ Nevertheless, at a critical temperature $T_{c}$ one finds that there is a solution $\Delta\left(\omega_{n}\right)$ of the linearized BCS gap equation

$$
-T_{c} \sum_{n^{\prime}} \frac{\pi N(0) \Gamma\left(\omega_{n}-\omega_{n^{\prime}}\right)}{\left|\omega_{n^{\prime}}\right|} \Delta\left(\omega_{n^{\prime}}\right)=\Delta\left(\omega_{n}\right)
$$

This is because, while $\Gamma\left(\omega_{m}\right)$ is a positive function of $\omega_{m}$, it increases over an energy scale set by the characteristic phonon frequency $\Omega$. In this case, the pair scattering strength is large and positive for processes in which a pair is scattered from a smaller Matsubara frequency $\omega_{n^{\prime}}$ to a larger one $\omega_{n}$ such that $\left|\omega_{n}-\omega_{n^{\prime}}\right|>\Omega$. Then if $\Delta\left(\omega_{n^{\prime}}\right)$ is positive, the gap equation (A4) can be satisfied provided $\Delta\left(\omega_{n}\right)$ is negative as shown in Fig. 36b. This "sign-changing" frequency structure of the gap reflects the internal structure of a pair in which the electrons are dynamically correlated to avoid the "instantaneous" screened Coulomb interaction while taking advantage of the retarded phonon mediated attraction.

Another way to see that $\Gamma\left(\omega_{m}\right)$ describes an attractive pairing interaction is to replace $i \omega_{m}$ by $\omega+i \delta$ and take the Fourier transform to determine the time dependence of the 


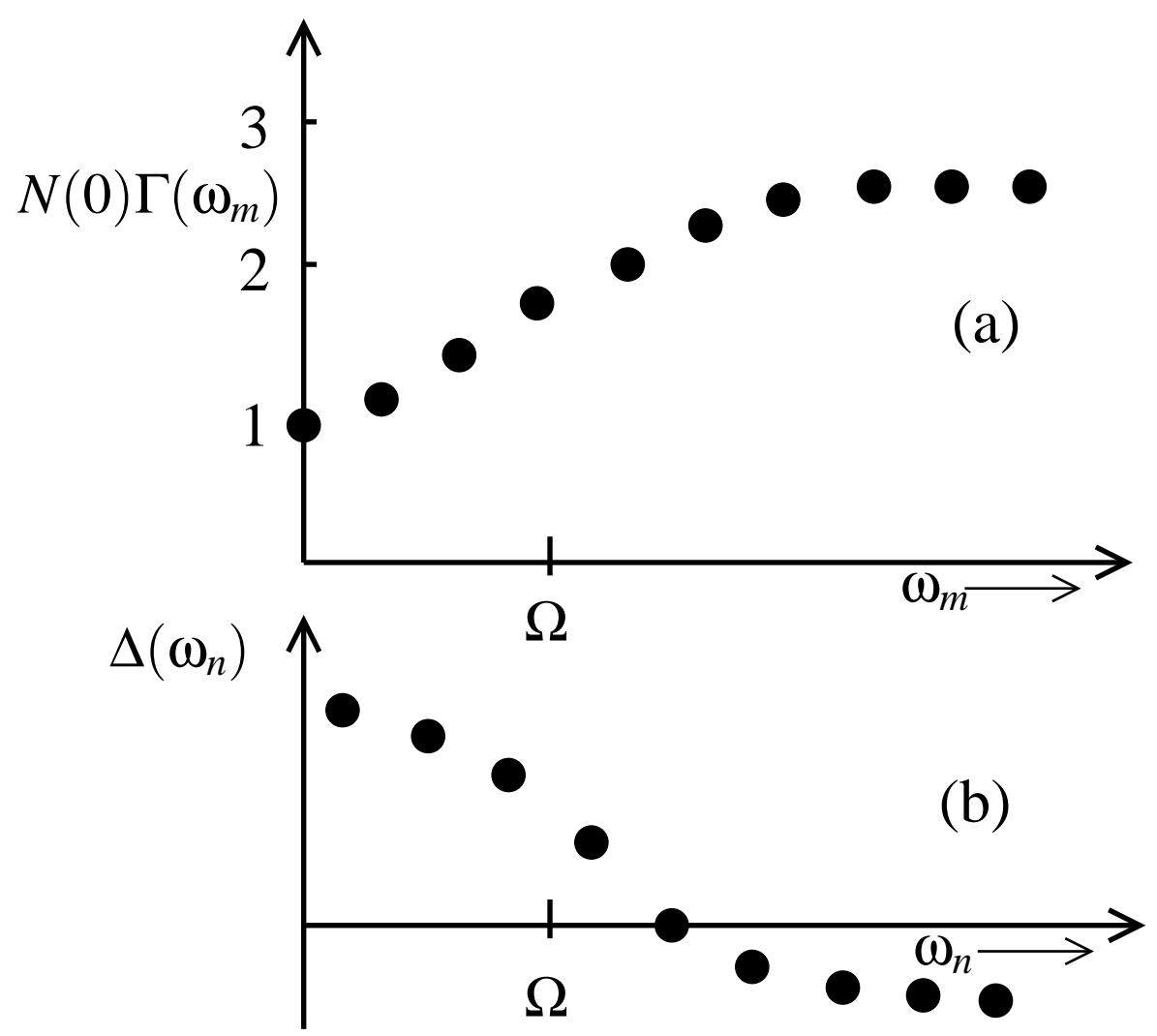

FIG. 36. (a) The vertex $\Gamma\left(\omega_{m}\right)$ multiplied by the single particle density of states $N(0)$ versus $\omega_{m}=2 m \pi T$. (b) The resulting gap $\Delta\left(\omega_{n}\right)$ associated with $\Gamma\left(\omega_{m}\right)$ versus $\omega_{n}=(2 n+1) \pi T$. The change in sign of $\Delta\left(\omega_{n}\right)$ is such that the gap Eq. (A4) can be satisfied even though $N(0) \Gamma\left(\omega_{m}\right)$ is positive for all $\omega_{m}$.

pairing interaction $\frac{189}{}$

$$
\Gamma(t)=\int \frac{d \omega}{2 \pi} e^{-i \omega t}\left(\frac{2|g|^{2} \Omega}{(\omega+i \delta)^{2}-\Omega^{2}}+V_{c}\right)
$$

then

$$
\operatorname{Re} \Gamma(t)=-|g|^{2} \sin \Omega t e^{-\delta t}+V_{c} \not \delta(t)
$$

with $\not(t)$ a broadened $\delta$-function of width $\mu_{F}^{-1}$. For a more general phonon spectrum peaked at $\Omega$ with a width $\Delta \Omega$, the first term decays for times larger than $\sim \Delta \Omega^{-1}$. Taking these features into account, Fig. 37 shows a schematic plot of $\operatorname{Re} \Gamma(t)$ in which one sees that the repulsive Coulomb interaction lasts for only a brief time of order the inverse of the Fermi energy while the attractive part of the interaction lasts for a much longer time set by the phonon spectral weight. 


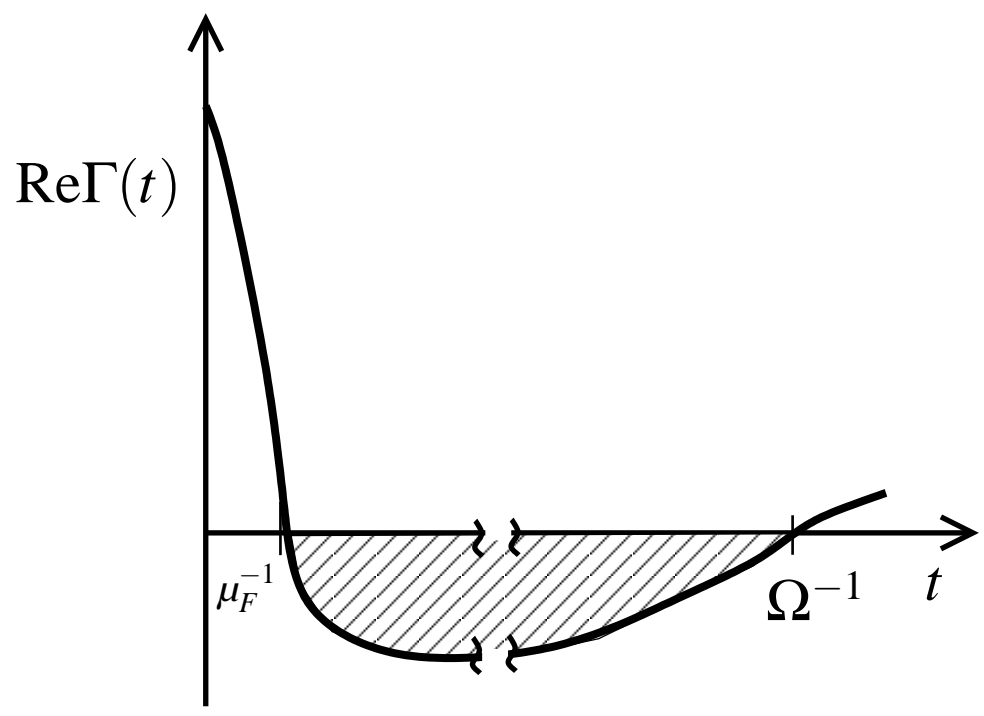

FIG. 37. Schematic plot of $\operatorname{Re} \Gamma(t)$ versus $t$. The interaction is repulsive for times less than of order $\mu_{F}^{-1}$ and then attractive for times between $\mu_{F}^{-1}$ and the inverse of a typical phonon frequency $\Omega^{-1}$.

\section{The Spin-Fluctuation Exchange Pairing Interaction}

In weak coupling, the leading RPA diagrams for the irreducible singlet particle-particle scattering vertex $\Gamma$ are shown in Fig. 38, These give
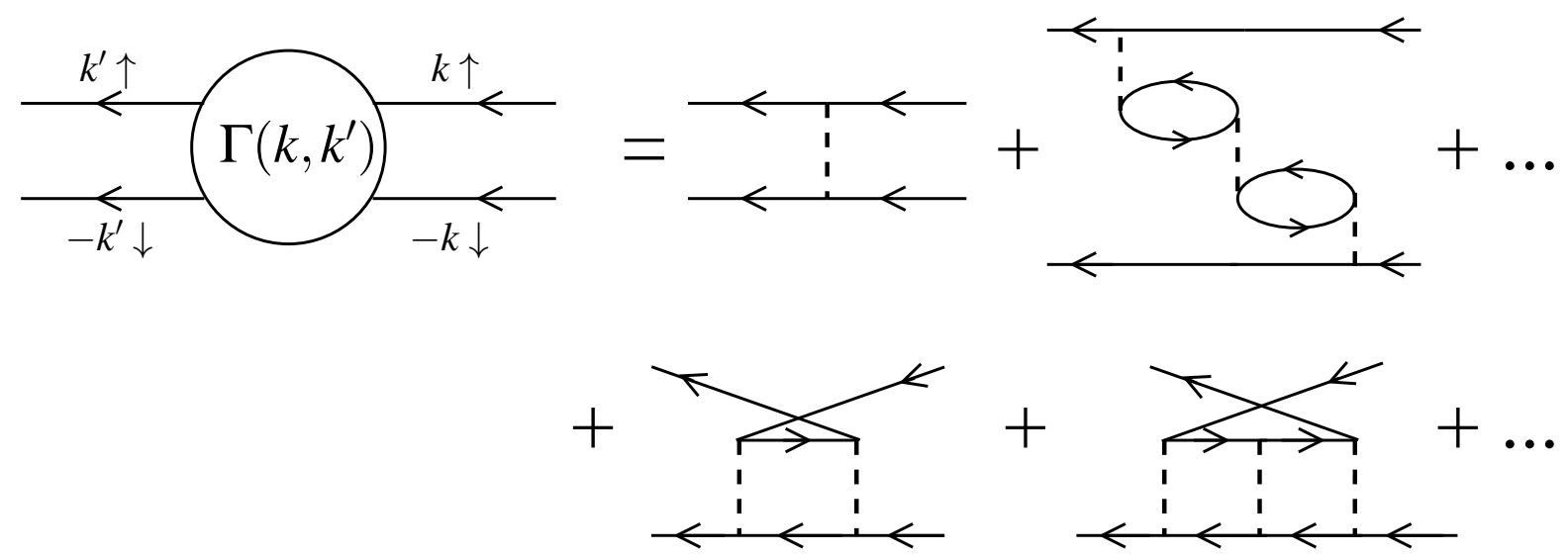

FIG. 38. The RPA diagrams for the Hubbard model pairing interaction. The solid lines are bare single-particle Green's functions and the dashed lines the interaction $U$. Here one clearly sees that the electrons which make up the spin-fluctuation pairing interaction are the same electrons that pair. 


$$
\Gamma\left(k, k^{\prime}\right)=\frac{U}{1-U^{2} \chi_{0}^{2}\left(k^{\prime}+k\right)}+\frac{U^{2} \chi_{0}\left(k^{\prime}-k\right)}{1-U \chi_{0}\left(k^{\prime}-k\right)}
$$

Here $k=\left(\mathbf{k}, i \omega_{n}\right)$ and $k^{\prime}=\left(\mathbf{k}^{\prime}, i \omega_{n^{\prime}}\right)$ and

$$
\chi_{0}\left(q, i \omega_{m}\right)=\frac{1}{N} \sum_{k} \frac{f\left(\varepsilon_{k+q}\right)-f\left(\varepsilon_{k}\right)}{i \omega_{m}-\varepsilon_{k+q}+\varepsilon_{k}} .
$$

For a single, even frequency pair, the gap function is even under $\mathbf{k}$ goes to $\mathbf{- k}$, so that one can replace $k^{\prime}+k$ by $k^{\prime}-k$ in the first term of Eq. (A7). Then, rearranging the terms in Eq. (A7) gives

$$
\Gamma\left(k, k^{\prime}\right)=\frac{3}{2} U^{2} \frac{\chi_{0}\left(k^{\prime}-k\right)}{1-U \chi_{0}\left(k^{\prime}-k\right)}+\frac{U^{2}}{2} \frac{\chi_{0}\left(k^{\prime}-k\right)}{1+U \chi_{0}\left(k^{\prime}-k\right)}+U .
$$

The first term is the contribution of the spin fluctuations with $\chi_{0}\left(1-U \chi_{0}\right)^{-1}$ the RPA spin susceptibility. The second term represents the charge fluctuations and $U$ is the onsite Coulomb interaction. This interaction was first used by Berk and Schrieffer to describe the depression of $T_{c}$ due to spin-fluctuations for $s$-wave superconductivity in $\mathrm{Pd}$.

For the 2D Hubbard model doped near half-filling, the dominant contribution to $\Gamma$ comes from the first term which peaks near $(\pi, \pi)$ reflecting the short range antiferromagnetic correlations. A plot of $\Gamma(q, 0)$ versus momentum transfer $q$ is given in Fig. 39 for $q$ along a path in the Brillouin zone shown in the inset. This interaction is positive for all momentum transfers. Therefore, for there to be a transition to a superconducting state, the gap function $\Delta(k)$ must have a change of sign on the Fermi surface in order to satisfy the BCS equation.

$$
\Delta(k)=-\frac{1}{N} \sum_{k^{\prime}} \frac{\Gamma\left(k-k^{\prime}\right) \Delta\left(k^{\prime}\right)}{2 \varepsilon_{k}} \tanh \left(\beta_{c} \varepsilon_{k} / 2\right) .
$$

For the nearly half-filled 2D Hubbard model, Eq. (A10) leads to the well-known $\Delta(k)=$ $\Delta_{0}\left(\cos k_{x}-\cos k_{y}\right) d_{x^{2}-y^{2}}$ gap. In this case, $(k \uparrow,-k \downarrow)$ pairs with $k$ near $(\pi, 0)$ which have a negative gap are strongly scattered by the antiferromagnetic spin fluctuations to $\left(k^{\prime} \uparrow,-k^{\prime} \downarrow\right)$ pairs with $k^{\prime}$ near $(0, \pi)$ which have a positive gap, satisfying Eq. (A10). This sign change in the momentum dependence of $\Delta(k)$ reflects an internal structure of a pair in which the electrons are spatially correlated such that they avoid occupying the same site while taking advantage of the non-local attractive regions of the interaction. It is a $d_{x^{2}-y^{2}}$ pair rather than an extended $s$-wave $\left(\cos k_{x}+\cos k_{y}\right)$ pair because it is made up from states near the nearly half-filled Fermi surface. This structure of the interaction is illustrated in Fig. 40, which shows the spatial Fourier transform of $\Gamma(q)$. Here one member of the pair is located 


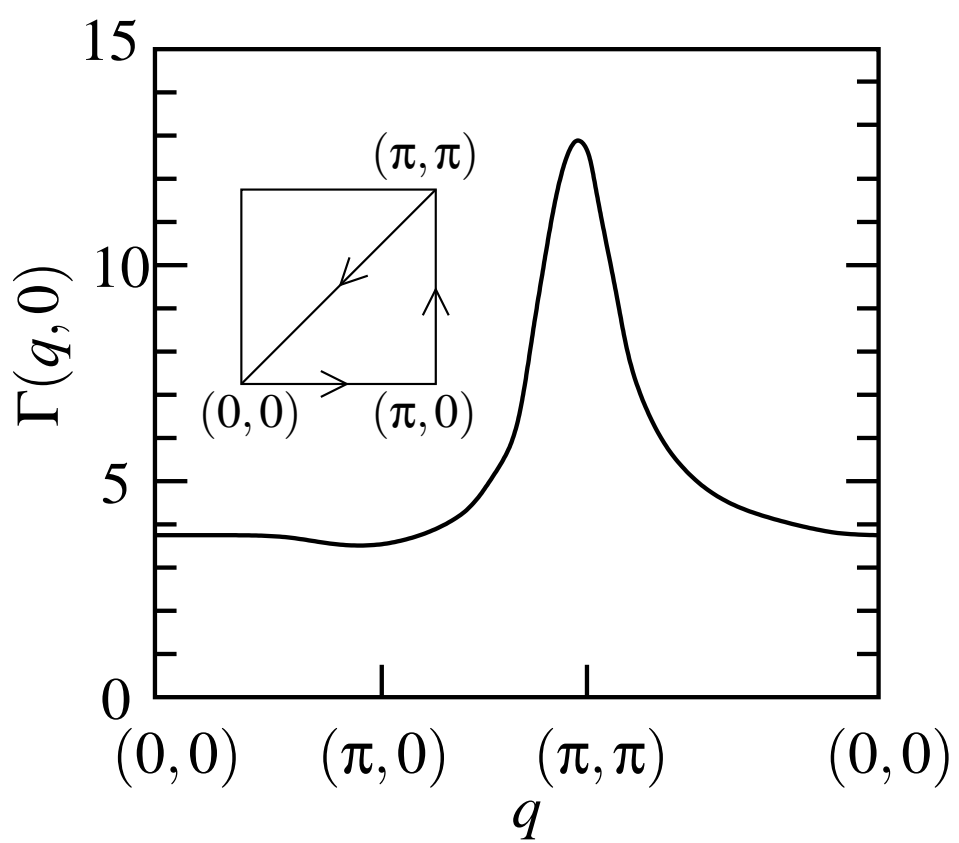

FIG. 39. $\Gamma(q, 0)$ versus $q$ for $q$ along a path in the Brillouin zone which is shown in the inset. An effective interaction that is peaked at a large momentum transfer is the origin of the unconventional superconductivity discussed in this review. Here $U=2 t, t^{\prime}=0,\langle n\rangle=0.87$ and $T=0.25 t$.

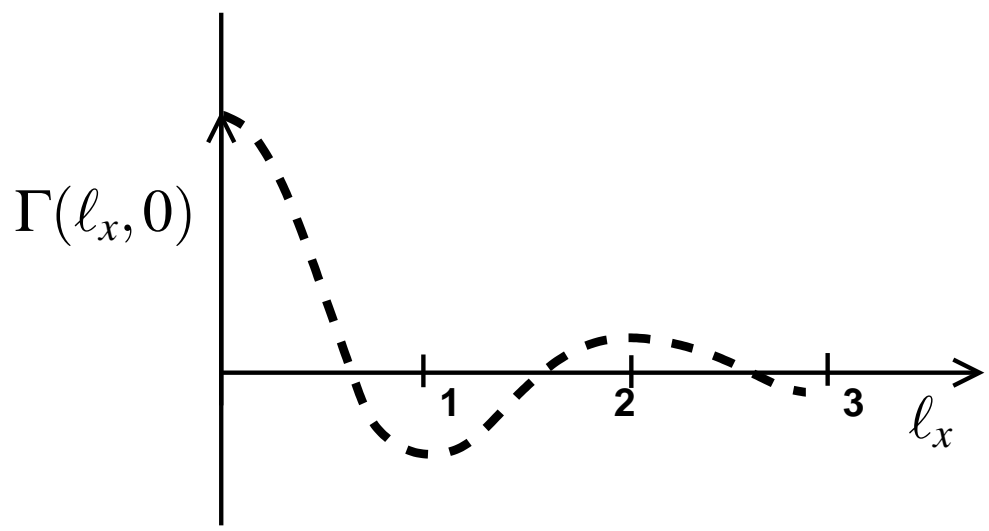

FIG. 40. The spatial Fourier transform $\Gamma\left(\ell_{x}, \ell_{y}=0\right)$ versus $\ell_{x}$. Here one member of a pair is at the origin and $\Gamma\left(\ell_{x}, 0\right)$ is the interaction energy when a second electron is added in a single state at site $\ell_{x}$.

at the origin and another at site $\left(\ell_{x}, 0\right)$.

Thus both the conventional and the unconventional superconductors have "sign changing 
gaps." For the conventional case this sign change occurs in the frequency dependence of the gap and reflects the dynamic correlations of the electrons which form the Cooper pairs. In the case of the unconventional superconductors, the sign change occurs in the momentum dependence of the gap and reflects the spatial correlations of the paired electrons. Naturally, there are also dynamic correlations since the spin-fluctuations are retarded and similarly in the phonon case there are some spatial correlations due to the momentum dependence of the electron-phonon interaction. However, the characteristic feature of the spin-fluctuation interaction is its momentum dependence which leads to a spatially non-local pairing interaction, while the characteristic feature of the phonon mediated pairing interaction is its frequency dependence which leads to a retarded pairing interaction. 


\section{ACKNOWLEDGMENTS}

This review article represents a more detailed account of talks given at the M2S09 conference and a subsequent CIFAR meeting. The author would especially like to thank CIFAR and the members of the Quantum Materials Program for providing an environment where ideas can be discussed, debated and criticized. I would like to acknowledge the close collaboration that I have enjoyed working with T.A. Maier, and the many useful discussions with A.D. Christianson, E. Dagotto, M. Lumsden, D. Mandrus, A. Moreo, H.A. Mook and D. Singh at ORNL. I would like to thank E. Berg, S. Graser, P. Hirschfeld, A.F. Kemper, S.A. Kivelson, S. Raghu and S.R. White for joint work and many discussions. I am grateful to Z. Fisk, R. Greene, W. Hanke, D. Hone, B. Keimer, K. Kuroki, T.M. Rice, S. Sachdev, Z.-X. Shen, L. Taillefer and J. Thompson for sharing their insights. Finally, I want to thank N. Ghimire, S. Graser, and R. Melko for help with some of the figures and express my gratitude to D.L. Ceder for her patience and dedication in putting this manuscript together. The author acknowledges the support of the Center for Nanophase Materials Science at ORNL, which is sponsored by the Division of Scientific User Facilities, U.S. DOE and thanks the Stanford Institute of Theoretical Physics for their hospitality.

* djs@physics.ucsb.edu

1 Z. Fisk, H.-R. Ott, and J. D. Thompson, Philosophical Magazine 89, 2111 (2009).

2 A brief account of this was given in the Proceedings of the M2S - IX Conference, Physica C 470, 51-54 (2010).

3 K. Bechgaard, C. S. Jacobsen, K. Mortensen, H. J. Pedersen, and N. Thorup, Solid State Commun. 33, 1119 (1980).

4 C. Bourbonnais and D. Jérome (Springer, Heidelberg, 2008) p. 257, arXiv:0904.0616.

5 L. Taillefer, Annual Review of Condensed Matter Physics 1, 51 (2010).

6 N. Doiron-Leyraud, P. Auban-Senzier, S. R. de Cotret, C. Bourbonnais, D. Jérome, K. Bechgaard, and L. Taillefer, Phys. Rev. B 80, 214531 (2009).

7 These illustrations were made by N. Ghimire using a CrystalMaker 8.5 software package. D.R. Harshman, A.T. Fiory and J.D. Dow, J. Phys: Condens. Matter 23, 295701 (2011) 
contains a useful tabulation of $T_{c}$ values.

8 H. Shishido, T. Shibauchi, K. Yasu, T. Kato, H. Kontani, T. Terashima, and Y. Matsuda, Science 327, 980 (2010).

9 G. Chen, S. Ohara, M. Hedo, Y. Uwatoko, K. Saito, M. Sorai, and I. Sakamoto, J. Phys. Soc. Japan 71, 2836 (2002).

10 C. Petrovic, P. G. Pagliuso, M. F. Hundley, R. Movshovich, J. L. Sarrao, J. D. Thompson, Z. Fisk, and P. Monthoux, J. Phys.: Condens. Matter 13, L337 (2001).

11 C. Petrovic, R. Movshovich, M. Jaime, P. G. Pagliuso, M. F. Hundley, J. L. Sarrao, Z. Fisk, and J. D. Thompson, Europhys. Lett. 53, 354 (2001).

12 H. Hegger, C. Petrovic, E. G. Moshopolou, M. F. Hundley, J. L. Sarrao, Z. Fisk, and J. D. Thompson, Phys. Rev. Lett. 84, 4986 (2000).

13 J. L. Sarrao, L. A. Morales, J. D. Thompson, B. L. Scott, G. R. Stewart, F. Wastin, J. Rebizant, P. Boulet, E. Colineau, and G. H. Lander, Nature (London) 420, 297 (2002).

14 F. Wastin, P. Boulet, J. Rebizant, E. Colineau, and G. H. Lander, J. Phys.: Condens. Matter 15, S2279 (2003).

15 J.-X. Zhu, P. H. Tobash, E. D. Bauer, F. Ronning, B. L. Scott, K. Haule, G. Kotliar, R. C. Albers, and J. M. Wills, Europhys. Lett. 97, 57001 (2012).

16 J. L. Wagner, B. A. Hunter, D. G. Hinks, and J. D. Jorgensen, Phys. Rev. B 51, 15407 (1995).

17 W. Chu, L. Gao, F. Chen, Z. J. Huang, R. L. Meng, and Y. Y. Xue, Nature 365, 323 (1993).

18 F. Goutenoire, P. Daniel, M. Hervieu, G. van Tendeloo, C. Michel, A. Maignan, and B. Raveau, Physica C 216, 243 (1993).

19 H. Takagi, R. J. Cava, M. Marezio, B. Batlogg, J. J. Krajewski, Peck, Jr., W. F., P. Bordet, and D. E. Cox, Phys. Rev. Lett. 68, 3777 (1992).

20 K. Yamada, K. Kurahashi, T. Uefuji, M. Fujita, S. Park, S.-H. Lee, and Y. Endoh, Phys. Rev. Lett. 90, 137004 (2003).

21 J. D. Jorgensen, P. G. Radaelli, D. G. Hinks, L. J. Wanger, S. Kikkawa, G. Er, and F. Kanamaru, Phys. Rev. B 47, 14654 (1993).

22 Y. Kamihara, H. Hiramatsu, M. Hirano, R. Kawamura, H. Yanagi, T. Kamiya, and H. Hosono, J. Am. Chem. Soc. 128, 10012 (2006).

23 Y. Kamihara, T. Watanabe, M. Hirano, and H. Hosono, J. Am. Chem. Soc. 130, 3296 (2008). 
24 C. de la Cruz, Q. Huang, J. W. Lynn, J. Li, Ratcliff, II, W., J. L. Zarestky, H. A. Mook, G. F. Chen, J. L. Luo, N. L. Wang, and P. Dai, Nature 453, 899 (2008).

25 O. Delaire, M. S. Lucas, A. M. dos Santos, A. Subedi, A. S. Sefat, M. A. McGuire, L. Mauger, J. A. Muñoz, C. A. Tulk, Y. Xiao, M. Somayazulu, J. Y. Zhao, W. Sturhahn, E. E. Alp, D. J. Singh, B. C. Sales, D. Mandrus, and T. Egami, Phys. Rev. B 81, 094504 (2010).

26 M. Rotter, M. Tegel, and D. Johrendt, Phys. Rev. Lett. 101, 107006 (2008).

27 H. Okabe, N. Takeshita, K. Horigane, T. Muranaka, and J. Akimitsu, Phys. Rev. B 81, 205119 (2010).

28 T. C. Ozawa and S. M. Kauzlarich, Sci. Technol. Adv. Mater. 9, 033003 (2008).

29 Z. A. Ren, W. Lu, J. Yang, W. Yi, X. L. Shen, C. Z. Li, G. C. Che, X. L. Dong, L. L. Sun, F. Zhou, and Z. X. Zhao, Chinese Phys. Lett. 25, 2215 (2008).

30 M. Rotter, M. Tegel, D. Johrendt, I. Schellenberg, W. Hermes, and R. Poettgen, Phys. Rev. B. 78, 020503 (2008).

31 A. S. Sefat, R. Y. Jin, M. A. McGuire, B. C. Sales, D. J. Singh, and D. Mandrus, Phys. Rev. Lett. 101, 117004 (2008).

32 Y. Mizuguchi, Y. Hara, K. Deguchi, S. Tsuda, T. Yamaguchi, K. Takeda, H. Kotegawa, H. Tou, and Y. Takano, Supercond. Sci. Technol. 23, 054013 (2010).

33 S. E. Sebastian, J. Gillett, N. Harrison, P. H. C. Lau, D. J. Singh, C. H. Mielke, and G. G. Lonzarich, J. Phys.: Condens. Matter 20, 422203 (2008).

34 A. I. Coldea, J. D. Fletcher, A. Carrington, J. G. Analytis, A. F. Bangura, J.-H. Chu, A. S. Erickson, I. R. Fisher, N. E. Hussey, and R. D. McDonald, Phys. Rev. Lett. 101, 216402 (2008).

35 A. I. Coldea, Phil. Trans. Royal Society A 368, 3503 (2010).

36 P. W. Anderson, Phys. Rev. 79, 350 (1950).

37 T. Hotta and K. Ueda, Phys. Rev. B 67, 104518 (2003).

38 T. Park, E. D. Bauer, and J. D. Thompson, Phys. Rev. Lett. 101, 177002 (2008).

39 L. D. Pham, T. Park, S. Maquilon, J. D. Thompson, and Z. Fisk, Phys. Rev. Lett. 97, 056404 (2006).

40 M. Nicklas, O. Stockert, T. Park, K. Habicht, K. Kiefer, L. D. Pham, J. D. Thompson, Z. Fisk, and F. Steglich, Phys. Rev. B 76, 052401 (2007). 
41 H. Shishido, R. Settai, D. Aoki, S. Ikeda, H. Nakawaki, N. Nakamura, T. Iizuka, Y. Inada, K. Sugiyama, T. Takeuchi, K. Kindo, T. C. Kobayashi, Y. Haga, H. Harima, Y. Aoki, T. Namiki, H. Sato, and Y. Ōnuki, J. Phys. Soc. Jpn. 71, 162 (2002).

42 V. J. Emery, S. A. Kivelson, and J. M. Tranquada, Proc. Natl. Acad. Sci. USA 96, 8814 (1999).

43 S. A. Kivelson and E. Fradkin, in Handbook of High Temperature Superconductivity, edited by J. R. Schrieffer and J. Brooks (Springer, 2007) Chap. 15.

44 N. P. Armitage, P. Fournier, and R. L. Green, Rev. Mod. Phys. 82, 2421 (2010).

45 R. M. Fernandes, D. K. Pratt, W. Tian, J. Zarestky, A. Kreyssig, S. Nandi, M. G. Kim, A. Thaler, N. Ni, P. C. Canfield, R. J. McQueeney, J. Schmalian, and A. I. Goldman, Phys. Rev. B 81, 140501 (2010).

46 J. Paglione and R. L. Greene, Nature Physics 6, 645 (2010).

47 A. B. Vorontsov, M. G. Vavilov, and A. V. Chubukov, Phys. Rev. B 79, 060508(R) (2009).

48 S. Nandi, M. G. Kim, A. Kreyssig, R. M. Fernandes, A. T. D. K. Pratt, N. Ni, S. L. Bud'ko, P. C. Canfield, J. Schmalian, R. J. McQueeney, and A. I. Goldman, Phys. Rev. Lett. 104, 057006 (2010).

49 Y. LaPlace, J. Bobroff, F. Rullier-Albenque, D. Colson, and A. Forget, Phys. Rev. B 80, 140501 (2009).

50 B. Khaykovich, S. Wakimoto, R. J. Birgeneau, M. A. Kastner, Y. S. Lee, P. Smeibidl, P. Vorderwisch, and K. Yamada, Phys. Rev. B 71, 220508(R) (2005).

51 B. Lake, H. M. Rønnow, N. B. Christensen, G. Aeppli, K. Lefmann, D. F. McMorrow, P. Vorderwisch, P. Smeibidl, N. Mangkorntong, T. Sasagawa, M. Nohara, H. Takagi, and T. E. Mason, Nature 415, 299 (2002).

52 E. Demler, S. Sachdev, and Y. Zhang, Phys. Rev. Lett. 87, 067202 (2001).

53 S. A. Kivelson, D.-H. Lee, E. Fradkin, and V. Oganesyan, Phys. Rev. B 66, 144516 (2002).

54 C. J. Halboth and W. Metzner, Phys. Rev. Lett. 85, 5162 (2000).

55 C. Honerkamp, M. Salmhofer, N. Furukawa, and T. M. Rice, Phys. Rev. B 63, 035109 (2001).

56 H. Zhai, F. Wang, and D.-H. Lee, Phys. Rev. B 80, 064517 (2009).

57 C. Platt, C. Honerkamp, and W. Hanke, New J. Phys. 11, 055058 (2009).

58 Q. Li, M. Hücker, G. D. Gu, A. M. Tsvelik, and J. M. Tranquada, Phys. Rev. Lett. 99, 067001 (2007). 
59 J. M. Tranquada, G. D. Gu, M. Hücker, Q. Jie, H.-J. Kang, R. Klingeler, Q. Li, N. Tristan, J. S. Wen, G. Y. Xu, Z. J. Xu, J. Zhou, and M. von Zimmermann, Phys. Rev. B 78, 174529 (2008).

60 E. Berg, E. Fradkin, E.-A. Kim, S. A. Kivelson, V. Oganesyan, J. M. Tranquada, and S. C. Zhang, Phys. Rev. Lett. 99, 127003 (2007).

61 A. Himeda, T. Kato, and M. Ogata, Phys. Rev. Lett. 88, 117001 (2002).

62 D. J. Scalapino and S. R. White, Phys. Rev. B 58, 8222 (1998).

63 H. Woo, P. Dai, S. M. Hayden, H. A. Mook, T. Dahm, D. J. Scalapino, T. G. Perring, and F. Doğan, Nat. Phys. 2, 600 (2006).

64 O. Stockert, J. Arndt, E. Faulhaber, C. Geibel, H. S. Jeevan, S. Kirchner, M. Loewenhaupt, K. Schmalzl, W. Schmidt, Q. Si, and F. Steglich, Nature Phys. 7, 119 (2011).

65 T. Imai, K. Ahilan, F. L. Ning, T. M. McQueen, and R. J. Cava, Phys. Rev. Lett. 102, 177005 (2009).

66 S. Wakimoto, H. Zhang, K. Yamada, I. Swainson, H. Kim, and R. J. Birgeneau, Phys. Rev. Lett. 92, 217004 (2004).

67 M. L. Tacon, G. Ghiringhelli, J. Chaloupka, M. M. Sala, V. Hinkov, M. W. Haverkort, M. Minola, M. Bakr, K. J. Zhou, S. Blanco-Canosa, C. Monney, Y. T. Song, G. L. Sun, C. T. Lin, G. M. D. Luca, M. Salluzzo, G. Khaliullin, T. Schmitt, L. Braicovich, and B. Keimer, Nature Phys. 7, 725 (2011).

68 R. Daou, N. Doiron-Leyraud, D. LeBoeuf, S. Y. Li, F. Laliberté, O. Cyr-Choinière, Y. J. Jo, L. Balicas, J.-Q. Yan, J.-S. Zhou, J. B. Goodenough, and L. Taillefer, Nature Phys. 5, 31 (2009).

69 N. Doiron-Leyraud, S. R. de Cotret, A. Sedeki, C. Bourbonnais, L. Taillefer, P. Auban-Senzier, D. Jérome, and K. Bechgaard, Eur. Phys. J. B 78, 23 (2010).

70 S. A. Hartnoll, D. M. Hofman, M. A. Metlitski, and S. Sachdev, Phys. Rev. B 84, 125115 (2011).

71 K. Jin, N. P. Butch, K. Kirshenbaum, J. Paglione, and R. L. Greene, Nature 476, 73 (2011).

72 J. Paglione, M. Tanatar, D. Hawthorn, E. Boaknin, R. W. Hill, F. Ronning, M. Sutherland, L. Taillefer, C. Petrovic, and P. C. Canfield, Phys. Rev. Lett. 91, 246405 (2003).

73 Q. Si and F. Steglich, Science 329, 1161 (2010). 
74 J. Rossat-Mignod, L. P. Regnault, C. Vettier, P. Bourges, P. Burlet, J. Bossy, J. Y. Henry, and G. Lapertot, Physica C 185, 86 (1991).

75 H. A. Mook, M. Yethiraj, G. Aeppli, T. E. Mason, and T. Armstrong, Phys. Rev. Lett. 70, 3490 (1993).

76 H. F. Fong, B. Keimer, P. W. Anderson, D. Reznik, F. Dogan, and I. Aksay, Phys. Rev. Lett. $\mathbf{7 5}, 316(1995)$.

77 H. F. Fong, Y. Sidis, L. P. Regnault, A. Ivanov, G. D. Gu, N. Koshizuka, and B. Keimer, Nature 398, 588 (1999).

78 C. Stock, C. Broholm, J. Hudis, H. J. Kang, and C. Petrovic, Phys. Rev. Lett. 100, 087001 (2008).

79 A. D. Christiansen, E. A. Goremychkin, R. Osborn, S. Rosenkranz, M. D. Lumsden, C. D. Malliakas, I. S. Todorov, H. Claus, D. Y. Chung, M. G. Kanatzidis, R. I. Bewley, and T. Guidi, Nature 456, 930 (2008).

80 J. T. Park, G. Friemel, Y. Li, J.-H. Kim, V. Tsurkan, J. Deisenhofer, H. von Nidda, A. Loidl, A. Ivanov, B. Keimer, and D. S. Inosov, Phys. Rev. Lett. 107, 177005 (2011).

81 D. S. Inosov, J. T. Park, P. Bourges, D. L. Sun, Y. Sidis, A. Schneidewind, K. Hradil, D. Haug, C. T. Lin, B. Keimer, and V. Hinkov, Nature of Physics 6, 178 (2010).

82 M. D. Lumsden and A. D. Christiansen, J. Phys.:Condens. Matter 22, 203203 (2010).

83 K. Terashima, Y. Sekiba, J. H. Bowen, K. Nakayama, T. Kawahara, T. Sato, P. Richard, Y.-M. Xu, L. J. Li, G. H. Cao, Z.-A. Xu, H. Ding, and T. Takahashi, Proc. Nat. Acad. Sci. USA 106, $7330(2009)$.

84 D. S. Inosov, V. B. Zabolotnyy, A. Koitzsch, M. Knupfer, B. Büchner, M. S. Viazovska, G. L. Sun, V. Hinkov, A. V. Boris, C. T. Lin, B. Keimer, A. Varykhalov, A. A. Kordyuk, and S. V. Borisenko, Phys. Rev. B 79, 054517 (2009).

85 N. Bulut, D. J. Scalapino, and R. T. Scalettar, Phys. Rev. B 45, 5577 (1992).

86 P. Monthoux and D. J. Scalapino, Phys. Rev. Lett. 72, 1874 (1994).

87 I. I. Mazin, D. J. Singh, M. D. Johannes, and M.-H. Du, Phys. Rev. Lett. 101, 057003 (2008).

88 P. J. Hirschfeld, M. M. Korshunov, and I. I. Mazin, Rep. Prog. Phys. 74, 124508 (2011).

89 J. Zaanen, G. A. Sawatzky, and J. W. Allen, Phys. Rev. Lett. 55, 418 (1985).

90 O. Gunnarsson, O. K. Andersen, O. Jepsen, and J. Zaanen, Phys. Rev. B 39, 1708 (1989).

91 T. Miyake, K. Nakamura, R. Arita, and M. Imada, J. Phys. Soc. Jpn. 79, 044705 (2010). 
92 V. Vildosola, L. Pourovskii, R. Arita, S. Biermann, and A. Georges, Phys. Rev. B 78, 064518 (2008).

93 K. Miyake, S. Schmitt-Rink, and C. M. Varma, Phys. Rev. B 34, 6554 (1986).

94 D. J. Scalapino, Loh, Jr., E., and J. E. Hirsch, Phys. Rev. B 34, 8190 (1986).

95 P. Monthoux, A. Balatsky, and D. Pines, Phys. Rev. Lett. 67, 3448 (1991).

96 S. Graser, T. A. Maier, P. J. Hirschfeld, and D. J. Scalapino, New J. Phys. 11, 025016 (2009).

97 P. W. Anderson, Science 235, 1196 (1987).

98 G. Kotliar and J. Liu, Phys. Rev. B 38, 5142 (1988).

99 P. W. Anderson, P. A. Lee, M. Randeria, T. M. Rice, N. Trivedi, and F. C. Zhang, J. Phys. Condes. Matter 16, R755 (2004).

100 N. E. Bickers, D. J. Scalapino, and S. R. White, Phys. Rev. Lett. 62, 961 (1989).

101 T. Dahm and L. Tewordt, Phys. Rev. B 52, 1297 (1995).

102 K. Kuroki, R. Arita, and H. Aoki, Phys. Rev. B 60, 9850 (1999).

103 T. Moriya and K. Ueda, Rep. Prog. Phys. 66, 1299 (2003).

104 A.-M. Tremblay, Theoretical Methods for Strongly Correlated Systems, edited by A. Avella and F. Mancini (Springer Valley, 2011).

105 P. Coleman, Phys. Rev. B 29, 3035 (1984).

106 A. E. Ruckenstein, P. J. Hirschfeld, and J. Appel, Phys. Rev. B 36, 857 (1987).

107 G. Kotliar and J. Liu, Phys. Rev. Lett. 61, 1784 (1988).

108 R. Blankenbecker, D. J. Scalapino, and R. L. Sugar, Phys. Rev. D 24, 2278 (1981).

109 J. E. Hirsch, Phys. Rev. 31, 4403 (1985).

110 T. Paiva, R. T. Scalettar, C. Huscroft, and A. K. McMahan, Phys. Rev. B 63, 125116 (2001).

111 C. Gros, Phys. Rev. B 38, 931 (1988).

112 A. Paramekanti, M. Randeria, and N. Trivedi, Phys. Rev. B 70, 054504 (2004).

113 M. Ogata and H. Fukuyama, Rep. Prog. Phys. 71, 036501 (2008).

114 G. Kotliar, S. Savrsov, G. Palsson, and G. Biroli, Phys. Rev. Lett. 87, 186401 (2001).

115 M. Jarrell, T. Maier, C. Huscroft, and S. Moukouri, Phys. Rev. B 64, 195130 (2001).

116 M. Potthoff, M. Aichhorn, and C. Dahnken, Phys. Rev. Lett. 91, 206402 (2003).

117 S. R. White, Phys. Rev. B 48, 10345 (1993).

118 M. R. Norman, J. Lin, and A. J. Millis, Phys. Rev. B 81, 180513(R) (2010).

119 H. Yao, D.-H. Lee, and S. A. Kivelson, Phys. Rev. B 84, 012507 (2011). 
120 E. G. Moon and S. Sachdev, Phys. Rev. B 82, 104516 (2010).

121 J. M. Tranquada, N. Ichikawa, and S. Uchida, Phys. Rev. B 59, 14712 (1999).

122 I. Tomeno, T. Machi, K. Tai, N. Koshizuka, S. Kambe, Y. U. A. Hayashi, and H. Yasuoka, Phys. Rev. B 49, 15327 (1994).

123 K.-Y. Yang, T. M. Rice, and F. C. Zhang, Phys. Rev. B 73, 174501 (2006).

124 S. Sachdev, Physica C 470, 54 (2010).

125 J. Hubbard, Proc. Roy. Soc. A 276, 238 (1963).

126 J. Oitmaa and D. D. Betts, Can. J. Phys. 56, 897 (1978).

127 S. Chakravarty, B. I. Halperin, and D. Nelson, Phys. Rev. Lett. 60, 1057 (1988).

128 D. P. Arovas and A. Auerbach, Phys. Rev. B 38, 316 (1988).

129 S. Raghu, S. A. Kivelson, and D. J. Scalapino, Phys. Rev. B 81, 224505 (2010).

130 J. E. Hirsch and R. M. Fye, Phys. Rev. Lett 56, 2521 (1986).

131 M. Salmhofer and C. Honerkamp, Prog. Theor. Physics 105, 1 (2001).

132 A. Lauchli, C. Honerkamp, and T. M. Rice, Phys. Rev. Lett. 92, 037006 (2009).

133 D. Senechal, P.-L. Lavertu, M.-A. Marois, and A.-M. S. Tremblay, Phys. Rev. Lett. 94, 156404 (2005).

134 M. Aichhorn, E. Arrigoni, M. Potthoff, and W. Hanke, Phys. Rev. B 74, 024508 (2006).

135 S. Okamoto, D. Sénéchal, M. Civelli, and A.-M. S. Tremblay, Phys. Rev. B 82, 180511(R) (2010).

136 A. Macridin, M. Jarrell, T. Maier, P. R. C. Kent, and E. D'Azevedo, Phys. Rev. Lett. 97, 036401 (2006).

137 S.-Q. Su and T. A. Maier, Phys. Rev. B 84, 220506(R) (2011).

138 D. Betts, H. Lin, and J. Flynn, Can. J. Phys. 77, 353 (1999).

139 T. A. Maier, M. Jarrell, T. C. Schulthess, P. R. C. Kent, and J. B. White, Phys. Rev. Lett. 95, 237001 (2005).

140 S. Maiti and A. V. Chubukov, Phys. Rev. B 82, 214515 (2010).

141 R. M. Fernandes, A. V. Chubukov, J. Knolle, I. Eremin, and J. Schmalian, Phys. Rev. B 85, 024534 (2012).

142 R. M. Noack, D. J. Scalapino, and S. R. White, Physica C 270, 281 (1996).

143 E. Berg, S. A. Kivelson, and D. J. Scalapino, New J. Phys. 11, 085007 (2009).

144 S. R. White and D. J. Scalapino, Phys. Rev. Lett. 91, 136403 (2003). 
145 G. Hager, G. Wellein, E. Jeckelmann, and H. Fehske, Phys. Rev. B 71, 75108 (2005).

146 S. R. White and D. J. Scalapino, Phys. Rev. Lett. 81, 3227 (1998).

147 M. Fujita, H. Goka, K. Yamada, J. M. Tranquada, and L. P. Regnault, Phys. Rev. B 70, 104517 (2004).

148 B. Kyung, S. S. Kancharla, D. Senechal, A.-M. S. Tremblay, M. Civelli, and G. Kotliar, Phys. Rev. B 73, 165114 (2006).

149 M. Aichhorn, E. Arrigoni, Z. B. Huang, and W. Hanke, Phys. Rev. Lett. 99, 257002 (2007).

150 D. J. Scalapino, in Handbook of High Temperature Superconductivity, edited by J. Schrieffer and J. Brooks (Springer, 2007) Chap. 13.

151 S. S. Kancharla, B. Kyung, D. Senechal, M. Civelli, M. Capone, G. Kotliar, and A.-M. S. Tremblay, Phys. Rev. B 77, 184516 (2008).

152 B. Fauque, Y. Sidis, V. Hinkov, S. Pailhès, C. T. Lin, X. Chaud, and P. Bourges, Phys. Rev. Lett. 96, 197001 (2006).

153 A. Kaminski, S. Rosenkranz, H. M. Fretwell, J. C. Campuzano, Z. Li, H. Raffy, W. G. Cullen, H. You, C. G. Olson, C. M. Varma, and H. Hochst, Nature 416, 610 (2002).

154 D. C. Johnston, Adv. in Phys. 59, 803 (2010).

155 W. Malaeb, T. Yoshida, T. Kataoka, A. Fujimori, M. Kubota, K. Ono, H. Usui, K. Kuroki, R. Arita, H. Aoki, Y. Kamihara, M. Hirano, and H. Hosono, J. Phys. Soc. Jpn. 77, 093714 (2008).

156 S. Lebègue, Phys. Rev. B 75, 035110 (2007).

157 D. J. Singh and M.-H. Du, Phys. Rev. Lett. 100, 237003 (2008).

158 C. Cao, P. J. Hirschfeld, and H.-P. Cheng, Phys. Rev. B 77, 220506 (2008).

159 P. A. Lee and X.-G. Wen, Phys. Rev. B 78, 144517 (2008).

160 K. Kuroki, S. Onari, R. Arita, H. Usui, Y. Tanaka, H. Kontani, and H. Aoki, Phys. Rev. Lett. 101, 087004 (2008).

161 K. Suzuki, H. Usui, and K. Kuroki, J. Phys. Soc. Jpn. 80, 013710 (2011).

162 P. Gegenwart, Q. Si, and F. Steglich, Nature Phys. 4, 186 (2008).

163 S. Nair, O. Stockert, U. Witte, M. Nicklas, R. Schedler, K. Kiefer, J. D. Thompson, A. D. Bianchi, Z. Fisk, S. Wirth, and F. Steglich, Proc. Natl. Acad. Sci. USA 107, 9537 (2010).

164 S. Elgazzar, I. Opahle, R. Hayn, and P. M. Oppeneer, J. Mag. and Mag. Materials 290, 388 (2005). 
165 T. Maehira, T. Hotta, K. Ueda, and A. Hasegawa, Phys. Rev. Lett. 90, 207007 (2003).

166 D. Hall, E. C. Palm, T. P. Murphy, S. W. Tozer, Z. Fisk, U. Alver, R. G. Goodrich, J. L. Sarrao, P. G. Pagliuso, and T. Ebihara, Phys. Rev. B 64, 212508 (2001).

167 H. Shishido, A. F. Bangura, A. I. Coldea, S. Tonegawa, K. Hashimoto, S. Kasahara, P. M. C. Rourke, H. Ikeda, T. Terashima, R. Settai, Y. Ōnuki, D. Vignolles, C. Proust, B. Vignolle, A. McCollam, Y. Matsuda, T. Shibauchi, and A. Carrington, Phys. Rev. Lett. 104, 057008 (2010).

168 T. Terashima, M. Kimata, N. Kurita, H. Satsukawa, A. Harada, K. Hazama, M. Imai, A. Sato, K. Kihou, C.-H. Lee, H. Kito, H. Eisaki, A. Iyo, T. Saito, H. Fukazawa, Y. Kohori, H. Harima, and S. Uji, J. Phys. Soc. Japan 79, 053702 (2010).

169 M. R. Norman, Physics 3, 86 (2010).

170 N. E. Hussey, M. Abdel-Jawad, A. Carrington, A. P. Mackenzie, and L. Balicas, Nature 425, $814(2003)$.

171 M. Platé, J. D. F. Mottershead, I. S. Elfimov, D. C. Peets, R. Liang, D. A. Bonn, W. N. Hardy, S. Chiuzbaian, M. Falub, M. Shi, L. Patthey, and A. Damascelli, Phys. Rev. Lett. 95, 077001 (2005).

172 B. Vignolle, A. Carrington, R. A. Cooper, M. M. J. French, A. P. Mackenzie, C. Jaudet, D. Vignolles, C. Proust, and N. E. Hussey, Nature 455, 952 (2008).

173 R. Liang, D. A. Bonn, and W. N. Hardy, Physica C 336, 57 (2000).

174 N. Doiron-Leyraud, C. Proust, D. LeBoeuf, J. Levallois, J.-B. Bonnemaison, R. Liang, D. A. Bonn, W. N. Hardy, and L. Taillefer, Nature 447, 565 (2007).

175 C. Jaudet, D. Vignolles, A. Audouard, J. Levallois, D. LeBoeuf, N. Doiron-Leyraud, B. Vignolle, M. Nardone, A. Zitouni, R. Liang, D. A. Bonn, W. N. Hardy, L. Taillefer, and C. Proust, Phys. Rev. Lett. 100, 187005 (2008).

176 S. E. Sebastian, N. Harrison, E. Palm, T. P. Murphy, C. H. Mielke, R. Liang, D. A. Bonn, W. N. Hardy, and G. G. Lonzarich, Nature 454, 200 (2008).

177 D. LeBoeuf, N. Doiron-Leyraud, J. Levallois, R. Daou, J.-B. Bonnemaison, N. E. Hussey, L. Balicas, B. J. Ramshaw, R. Liang, D. A. Bonn, W. N. Hardy, S. Adachi, C. Proust, and L. Taillefer, Nature 450, 533 (2007).

178 J. Chang, R. Daou, C. Proust, D. LeBoeuf, N. Doiron-Leyraud, F. Laliberté, B. Pingault, B. J. Ramshaw, R. Liang, D. A. Bonn, W. N. Hardy, H. Takagi, A. B. Antunes, I. Sheikin, 
K. Behnia, and L. Taillefer, Phys. Rev. Lett. 104, 057005 (2010).

179

180

181

182 and M.-H. Julien, Nature 477, 191 (2011).

183

R. Daou, F. Laliberté, O. Cyr-Choiniére, J. Chang, Y. J. Jo, L. Balicas, R. Liang, D. A. Bonn, W. N. Hardy, C. Proust, and L. Taillefer, Phys. Rev. B 83, 054506 (2011).

184 F. Laliberté, J. Chang, N. Doiron-Leyraud, E. Hassinger, R. Daou, M. Rondeau, B. J. Ramshaw, R. Liang, D. A. Bonn, W. N. Hardy, S. Pyon, T. Takayama, H. Takagi, I. Sheikin, L. Malone, C. Proust, K. Behnia, and L. Taillefer, Nature Comm. 2, 432 (2011).

185 J. M. Tranquada, B. J. Sternlieb, J. D. Axe, Y. Nakamura, and S. Uchida, Nature 375, 561 (1995).

186 R. Daou, J. Chang, D. LeBoeuf, O. Cyr-Choinière, F. Laliberté, N. Doiron-Leyraud, B. J. Ramshaw, R. Liang, D. A. Bonn, W. N. Hardy, and L. Taillefer, Nature Phys. 463, 519 (2010).

187 R.-H. He, M. Hashimoto, H. Karapetyan, J. D. Koralek, J. P. Hinton, J. P. Testaud, V. Nathan, Y. Yoshida, H. Yao, K. Tanaka, W. Meevasana, R. G. Moore, D. H. Lu, S.-K. Mo, M. Ishikado, H. Eisaki, Z. Hussain, T. P. Devereaux, S. A. Kivelson, J. Orenstein, A. Kapitulnik, and Z.-X. Shen, Science 331, 1579 (2011).

188 S. E. Sebastian, G. G. Longarich, and N. Harrison, (2011), arXiv:1112.1373.

189 D. J. Scalapino, in Proceedings of the R. L. Orbach Inaugural Symposium, edited by W. P. Beyermann, N. L. Liu, and D. E. MacLaughlin (World Scientific, Singapore, 1994) pp. 155164.

190 W. L. McMillan and J. M. Rowell, Phys. Rev. Lett. 14, 108 (1965).

191 B. Farnworth and T. Timusk, Phys. Rev. B 3, 305 (1971).

192 A. Damascelli, Z. Hussain, and Z.-X. Shen, Rev. Mod. Phys. 75, 473 (2003).

193 J. C. Campuzano, M. R. Norman, and M. Randeria, "Physics of superconductors," (Springer, Berlin, Heidelberg, New York, 2004) pp. 167-273. 
194 T. Valla, T. E. Kidd, W.-G. Yin, G. D. Gu, P. D. Johnson, Z.-H. Pan, and A. V. Fedorov, Phys. Rev. Lett. 98, 167003 (2007).

195 A. A. Kordyuk, V. B. Zabolotnyy, D. V. Evtushinsky, D. S. Inosov, T. K. Kim, B. Büchner, and S. V. Borisenko, Eur. Phys. J. ST 188, 153 (2010).

196 J. H. Yun, J. M. Bok, H.-Y. Choi, W. Zhang, X. J. Zhou, and C. M. Varma, Phys. Rev. B 84, 104521 (2011).

197 D. J. Van Harlingen, Rev. Mod. Phys. 67, 515 (1995).

198 C. C. Tsuei and J. R. Kirtley, Rev. Mod. Phys. 72, 969 (2000).

199 T. Hanaguri, S. Niitaka, K. Kuroki, and H. Takagi, Science 328, 474 (2010).

200 B. Muschler, W. Prestel, E. Schachinger, J. P. Carbotte, R. Hackl, S. Ono, and Y. Ando, J. Phys: Condens. Mat. 22, 375702 (2010).

201 S. Caprara, C. D. Castro, B. Muschler, W. Prestel, R. Hackl, M. Lambacher, A. Erb, S. Komiya, Y. Ando, and M. Grilli, Phys. Rev. B 84, 054508 (2011).

202 M. Sutherland, D. G. Hawthorn, R. W. Hill, F. Ronning, S. Wakimoto, H. Zhang, C. Proust, E. Boaknin, C. Lupien, L. Taillefer, R. Liang, D. A. Bonn, W. N. Hardy, R. Gagnon, N. E. Hussey, T. Kimura, M. Nohara, and H. Takagi, Phys. Rev. B 67, 174520 (2003).

203 J. Lee, K. Fujita, K. McElroy, J. A. Slezak, M. Wang, Y. Aiura, H. Bando, M. Ishikado, T. Masui, J.-X. Zhu, A. V. Balatsky, H. Eisaki, S. Uchida, and J. C. Davis, Nature (London) 442, 546 (2006).

204 A. N. Pasupathy, A. Pushp, K. K. Gomes, C. V. Parker, J. Wen, Z. Xu, G. Gu, S. Ono, Y. Ando, and A. Yazdani, Science 320, 196 (2008).

205 N. Jenkins, Y. Fasano, C. Berthod, I. Maggio-Aprile, A. Piriou, E. Giannini, B. W. Hoogenboom, C. Hess, T. Cren, and Ø. Fischer, Phys. Rev. Lett. 103, 227001 (2009).

206 O. Ahmadi, L. Coffey, J. F. Zasadzinski, N. Miyakawa, and L. Ozyuzer, Phys. Rev. Lett. 106, 167005 (2011).

207 D. N. Basov and T. Timusk, Rev. Mod. Phys. 77, 721 (2005).

208 E. van Heumen, E. Muhlethaler, A. B. Kuzmenko, H. Eisaki, W. Meevasana, M. Greven, and D. van der Marel, Phys. Rev. B 79, 184512 (2009).

209 J. P. Carbotte, T. Timusk, and J. Hwang, Rep. Prog. Phys. 74, 066501 (2011).

210 X. J. Zhou, T. Cuk, T. Devereaux, N. Nagaosa, and Z.-X. Shen, in Handbook of HighTemperature Superconductivity, edited by J. R. Schrieffer and J. Brooks (Springer, 2007) 
Chap. 3.

211 J. M. Bok, J. H. Yun, H.-Y. Choi, W. Zhang, X. J. Zhou, and C. M. Varma, Phys. Rev. B 81, $174516(2010)$.

212 Y. Li, V. Balédent, G. Yu, N. Barišic', K. Hradil, R. A. Mole, Y. Sidis, P. Steffens, X. Zhao, P. Bourges, and M. Greven, Nature 468, 283 (2010).

213 C. Giannetti, F. Cilento, S. D. Conte, G. Coslovich, G. Ferrini, H. Molegraaf, M. Raichle, R. Liang, H. Eisaki, M. Greven, A. Damascelli, D. van der Marel, and F. Parmigiani, Nature Comm. 2, 353 (2011).

214 P. R. C. Kent, M. Jarrell, T. A. Maier, and T. Pruschke, Phys. Rev. B 72, 060411 (2005).

215 S. Fuchs, E. Gull, L. Pollet, E. Burovski, E. Kozik, T. Pruschke, and M. Troyer, Phys. Rev. Lett. 106, 030401 (2011).

216 T. A. Maier, M. S. Jarrell, and D. J. Scalapino, Phys. Rev. Lett. 96, 47005 (2006).

217 Just as the electron-phonon interaction strength is characterized by $\int \frac{d \omega}{\pi}\left|g_{q}\right|^{2} \frac{\operatorname{Im} \mathcal{D}(q, \omega)}{\omega}=$ $\left|g_{q}\right|^{2} \operatorname{Re} \mathcal{D}(q, 0)=\frac{-2\left|g_{q}\right|^{2}}{\omega_{q}}$ and a cut-off frequency of order $\omega_{\mathcal{D}}$, the pairing interaction strength for the Hubbard model is given by $\Gamma\left(k, k^{\prime}\right)$ with $\omega_{n}=\omega_{n^{\prime}}=\pi T$. The cut-off in the Matsubara frequency is set by the spin-fluctuation spectrum as shown in Fig. 22 ,

218 B. Kyung, D. Senechal, and A.-M. S. Tremblay, Phys. Rev. B 80, 205109 (2009).

219 T. A. Maier, D. Poilblanc, and D. J. Scalapino, Phys. Rev. Lett. 100, 237001 (2008).

220 W. Hanke, M. L. Kiesel, M. Aichhorn, S. Brehn, and E. Arrigoni, Eur. Phys. J. ST 188, 15 (2010).

221 T. A. Maier, M. S. Jarrell, and D. J. Scalapino, Phys. Rev. B 74, 094513 (2006).

222 M. A. Metlitski and S. Sachdev, Phys. Rev. B 80, 075128 (2010).

223 A. Abanov, A. V. Chubukov, and A. M. Finkel'stein, Europhys. Lett. 54, 488 (2001).

224 T. A. Maier, M. Jarrell, and D. J. Scalapino, Phys. Rev. B 75, 134519 (2007).

225 T. Dahm, V. Hinkov, S. V. Borisenko, A. A. Kordyuk, V. B. Zabolotnyy, J. Fink, B. Büchner, D. J. Scalapino, W. Hanke, and B. Keimer, Nature Phys. 5, 217 (2009).

226 A. J. Millis, S. Sachdev, and C. M. Varma, Phys. Rev. B 37, 4875 (1988).

227 P. Monthoux and D. J. Scalapino, Phys. Rev. B 50, 10339 (1994).

228 C.-H. Pao and N. E. Bickers, Phys. Rev. Lett. 72, 1870 (1994).

229 R. T. Scalettar, J. W. Cannon, D. J. Scalapino, and R. L. Sugar, Phys. Rev. B 50, 13419 (1994). 
230 R. E. Hetzel, W. Linden, and W. Hanke, Phys. Rev. B 50, 4159 (1994).

231 K. Bouadim, G. G. Batrouni, F. Hébert, and R. T. Scalettar, Phys. Rev. B 77, 144527 (2008).

232 K. Kuroki, T. Kimura, and R. Arita, Phys. Rev. B 66, 184508 (2002).

233 A. I. Liechtenstein, I. I. Mazin, and O. K. Andersen, Phys. Rev. Lett. 74, 2303 (1995).

234 T. A. Maier and D. J. Scalapino, Phys. Rev. B 84, 180513(R) (2011).

235 S. Sachdev and B. Keimer, Physics Today 64, 29 (2011).

236 A. F. Kemper, T. A. Maier, S. Graser, H.-P. Cheng, P. J. Hirschfeld, and D. J. Scalapino, New J. Phys. 12, 073030 (2010).

237 S. R. White, D. J. Scalapino, R. L. Sugar, E. Y. Loh, J. E. Gubernatis, and R. T. Scalettar, Phys. Rev. B 40, 506 (1989).

238 G. Dopf, A. Muramatsu, and W. Hanke, Phys. Rev. B 41, 9264 (1990).

239 K. Kuroki, H. Usui, S. Onari, R. Arita, and H. Aoki, Phys. Rev. B 79, 224511 (2009).

240 A. V. Chubukov, Ann. Rev. of Cond. Matter Phys. 3, 5792 (2012).

241 H. Ikeda, R. Arita, and J. Kuneš, Phys. Rev. B 82, 024508 (2010).

242 D. Wang, Y. Wan, and Q.-H. Wang, Phys. Rev. Lett. 102, 197004 (2009).

243 S. Uebelacker and C. Honerkamp, Phys. Rev. B 85, 155122 (2012).

244 T. Takimoto, T. Hotta, and K. Ueda, J. Phys: Condens. Matter 15, S2087 (2003).

245 R. Thomale, C. Platt, W. Hanke, and B. A. Bernevig, Phys. Rev. Lett. 106, 187003 (2011).

246 C. W. Hicks, T. M. Lippman, M. E. Huber, J. G. Analytis, J.-H. Chu, A. S. Erickson, I. R. Fisher, and K. A. Moler, Phys. Rev. Lett. 103, 127003 (2009).

247 C. Platt, R. Thomale, and W. Hanke, Ann. Phys. (Berlin) 523, 638 (2011).

248 Spin-fluctuations are also believed to give rise to pairing in $\mathrm{Sr}_{2} \mathrm{RuO}_{4} \stackrel{280}{ }$. Here, Rice and Sigrist $\underline{281}$ have proposed that the pairing is associated with small momentum transfer ferromagnetic fluctuations while Raghu et al. $\frac{282}{2}$ have suggested that the pairing is driven by large momentum spin-fluctuations associated with the quasi-1D band structure of $\mathrm{Sr}_{2} \mathrm{RuO}_{4}$. If the latter mechanism is correct, one would group $\mathrm{Sr}_{2} \mathrm{RuO}_{4}$ with the class of superconductors discussed in this review.

249 F. Steglich, J. Aarts, C. D. Bredl, W. Lieke, D. Meschede, W. Franz, and H. Schäfer, Phys. Rev. Lett. 43, 1892 (1979).

250 D. Jérome, A. Mazaud, M. Ribault, and K. Bechgaard, J. Phys. (Paris) Lett. 41, L95 (1980). 251 V. J. Emery, Synth. Met. 13, 21 (1986). 
252 M. Cyrot, Solid State Comm. 60, 253 (1986).

253 D. J. Scalapino, Phys. Rep. 250, 329 (1995).

254 P. Monthoux, D. Pines, and G. Longarich, Nature 450, 20 (2007).

255 P. W. Anderson, Science Magazine 316, 1705 (2007).

256 D. J. Scalapino, Science (2007), http://www.sciencemag.org/content/316/5832/1705/reply.

257 M. R. Norman, Science 332, 196 (2011).

258 R. Thomale, C. Platt, W. Hanke, J. Hu, and B. A. Bernevig, Phys. Rev. Lett. 107, 117001 (2011).

259 V. I. Emery and S. A. Kivelson, Nature (London) 374, 434 (1995).

260 A. Abanov, A. V. Chubukov, and J. Schmalian, Adv. Phys. 52, 119 (2003).

261 M. A. Metlitski and S. Sachdev, New J. Phys. 12, 105007 (2010).

262 Y. J. Uemura, Physica B 404, 3195 (2009).

263 N. J. Curro, T. Caldwell, E. D. Bauer, L. A. Morales, M. J. Graf, Y. Bang, A. V. Balatsky, J. D. Thompson, and J. L. Sarrao, Nature 434, 622 (2005).

264 T. Takimoto, T. Hotta, T. Maehira, and K. Ueda, J. Phys: Condens. Matter 14, L369 (2002).

265 H. Usui and K. Kuroki, Phys. Rev. B 84, 024505 (2011).

266 E. Pavarini, I. Dasgupta, T. Saha-Dasgupta, O. Jepsen, and O. K. Andersen, Phys. Rev. Lett. 87, 047003 (2001).

267 Y. Ohta, T. Tohyama, and S. Maekawa, Phys. Rev. B 43, 2968 (1991).

268 H. Sakakibara, H. Usui, K. Kuroki, R. Arita, and H. Aoki, Phys. Rev. Lett. 105, 057003 (2010).

269 P. Monthoux and G. G. Lonzarich, Phys. Rev. B 63, 054529 (2001).

270 E. Arrigoni, E. Fradkin, and S. A. Kivelson, Phys. Rev. B 69, 214519 (2004).

271 E. Berg, D. Orgad, and S. A. Kivelson, Phys. Rev. B 78, 094509 (2008).

272 J. R. Schrieffer, Theory of Superconductivity (Benjamin, New York, 1964).

273 F. Marsiglio and J. P. Carbotte, in Superconductivity, Vol. 1, edited by K. Benneman and J. Ketterson (Springer-Verlag, Berlin, 2008) Chap. 3, pp. 73-162.

274 A. B. Migdal, Soviet Phys. JETP 7, 996 (1958).

275 G. M. Eliashberg, Soviet Phys. JETP 11, 696 (1960).

276 N. N. Bogolinkov, V. V. Tolmachev, and D. V. Shirkov, A New Method in the Theory of Superconductivity (Consultants Bureau, New York, 1959). 
277 P. Morel and P. W. Anderson, Phys. Rev. 125, 1263 (1962).

278 In numerical solutions of the Eliashberg equations it is convenient to cut off the frequency integrals at a frequency $\omega_{c}$ of order five times the Debye frequency and replace $\mu=N(0) V_{s}$ by a renormalized pseudo-potential $\mu^{*}=\mu\left(1+\mu \ln \left(\frac{\mu_{F}}{\omega_{c}}\right)\right)^{-1} \underline{276}, 277$. This renormalization takes into account the fact that by decreasing the energy cut-off from $\mu_{F}$ to $\omega_{c}$ one has eliminated Coulomb scattering processes which keep the electrons apart. The phonon mediated part of the interaction is unchanged since $\omega_{c}$ is well above the dynamic range of the phonons. From a renormalization point of view, as the cut-off frequency is reduced $-\frac{2|g|^{2} N(0)}{\Omega}+\mu^{*}$ becomes negative and one has an effective low energy theory with an attractive pairing interaction. In this appendix, we are looking at the dynamics that underlies this renormalization.

279 N. F. Berk and J. R. Schrieffer, Phys. Rev. Lett. 17, 433 (1966).

280 Y. Maeno, H. Hashimoto, K. Yoshida, S. Nishizaki, T. Fujita, J. G. Bednorz, and F. Lichtenberg, Nature 372, 532 (1994).

281 T. M. Rice and M. Sigrist, J. Phys. C 7, L643 (1996).

282 S. Raghu, A. Kapitulnik, and S. A. Kivelson, Phys. Rev. Lett. 105, 136401 (2010). 